Elucidating the Roles of Stearoyl-CoA Desaturase 1 in Adipocyte Fatty Acid

\title{
Metabolism and Cellular Function
}

\author{
by \\ Jessica Claire Ralston
}

\begin{abstract}
A Thesis
presented to

The University of Guelph

In partial fulfillment of requirements

for the degree of

Doctor of Philosophy

in
\end{abstract}

Human Health and Nutritional Sciences

Guelph, Ontario, Canada

(C) Jessica Claire Ralston, July, 2015 


\section{ABSTRACT \\ ELUCIDATING THE ROLES OF STEAROYL-COA DESATURASE 1 IN ADIPOCYTE FATTY ACID METABOLISM AND CELLULAR FUNCTION}

Jessica Claire Ralston

University of Guelph, 2015
Advisor:

Dr. David M. Mutch

Worldwide obesity rates have risen to epidemic proportions, with over 600 million obese individuals across the globe. These individuals are prone to obesity-related health complications including type 2 diabetes, hypertension, cancer and cardiovascular disease. Obesity also coincides with the expansion of adipose tissue (AT), which has an important role storing excess calories in the form of triacylglycerol (TAG) within adipocyte lipid droplets. The predominant fatty acids (FAs) comprising adipocyte TAGs are monounsaturated FAs (MUFAs), which are produced by stearoyl-CoA desaturase 1 (SCD1). Specifically, SCD1 converts saturated FAs palmitate (PA) and stearate (SA) into palmitoleate (PMA) and oleate (OA), respectively. Interestingly, whole-body SCD1-deficiency is associated with reduced lipogenesis and adiposity. This places SCD1 as a potential target for obesity therapies; however, our understanding of the mechanisms linking SCD1 with changes in adipocyte function remains unclear. This thesis provides important new insights into how SCD1 impacts adipocyte FA metabolism and cellular function. Using a specific SCD1 inhibitor, changes in lipid metabolism, global gene expression, inflammation, cellular stress and basal insulin signaling were assessed in 3T3-L1 adipocytes. Results demonstrated that SCD1 inhibition caused a reduction in TAGs and phospholipids, which coincided with the down-regulation of genes associated with the biosynthesis of these lipid fractions. Cellular diacylglyerols were increased with SCD1 inhibition and insulin signaling was partially impaired. In contrast, markers of cellular stress were unaltered. Furthermore, the 
FA composition of each lipid fraction was dramatically modified, with SCD1-inhibited adipocytes specifically up-regulating the elongation of PA to SA. Stable isotope tracer experiments revealed that this elongation was occurring via elongase 6. Additionally, reduced SCD1 activity in adipocytes exacerbated the effects of exogenous SA on markers or inflammation. Taken together, SCD1 activity has many indirect influences on adipocyte metabolism in addition to its role in FA desaturation. Importantly, SCD1 facilitates the storage of FAs in TAGs and the ability of adipocytes to handle exogenous FAs. SCD1 also prevents saturated FA and DAG accumulation, and preserves insulin signaling in adipocytes. Ultimately this thesis highlights the importance of SCD1 in the maintenance of adipocyte cellular function, and emphasizes the wide-ranging impact of SCD1 on adipocyte FA metabolism. 


\section{$\underline{\text { Acknowledgements }}$}

First and foremost, I want to thank my advisor Dr. David Mutch, who has been far more than a supervisor or mentor for me over the past 5-6 years. David, to you I owe more than I will ever be able to articulate or perhaps even fathom. The passion that you have for science and research is not only inspiring, but infectious. And the genuine care, support and devotion you've relentlessly given me have always been truly touching. Above all, no matter the circumstances or how far I fell, you were constantly there to catch me. Thank you for always knowing which questions to ask, for pushing me towards accomplishments I never knew were possible, and for the 'tough love' that I sometimes needed. I will always appreciate and treasure your friendship and the precious time that I have been able to spend under your wing.

A second thank you goes to my advisory committee, who are two remarkable scientists that I feel privileged to have worked with and to have learnt from. Dr. David Wright, I have always appreciated your thought-provoking insights and all of our scientific discussions. Dr. David Dyck, I have learnt a lot from you about science and life, thank you for your endless encouragement. I would also like to thank Dr. Catherine Mounier, Dr. Lindsay Robinson and Dr. Jeremy Simpson for their important and valued roles in my examination.

To the past and present members of the Mutch lab, many of you have had such a positive influence on this experience, and I want to thank all of you for keeping science so fun. A special mention must go to Dr. Flavia Badoud. Flavia, you have had an instrumental impact on my growth as a scientist, and I am so privileged to have your friendship. To Kaitlin Roke, you have continually been there with me during the highs and lows of science, and your ability to know when a good talk is needed always amazes me. I am so fortunate to have such a caring friend in 
you. To Nick Gaudio, George Payne, Steve Dragos, Maude Perreault and Sarthak Matravadia, I have shared a record number of lab laughs with each of you, thank you.

To ALL of the incredible, hard-working and brilliant individuals I have had the pleasure of meeting and working with in HHNS, thank you for making every day such a delight. The familial environment of this department is truly remarkable, and I am so thankful that I have been able to make so many wonderful friendships here. To Erin Connelly, Kaity Roke and Tara MacDonald, you have been the greatest support network I could ask for, and I am so excited to see the amazing accomplishments that all three of you will inevitably continue to make in life. Erin, you are an inspiring scientist and person. I have always admired your dedication, strength and intellect, and I cannot imagine my life without your friendship, love and shared sense of humour.

To my family, Alan and Shirley Ralston, Jonathan, Kate and Katherine, thank you for your unconditional love and support. You are all such wonderfully positive influences in my life, and have been a fundamental source of strength in this process. Finally, a special thank you goes to Adam, who tolerated and endured more than anyone, and that has meant so much to me. I can't wait to see what life has in store for us next. 
TABLE OF CONTENTS ................................................................... vi

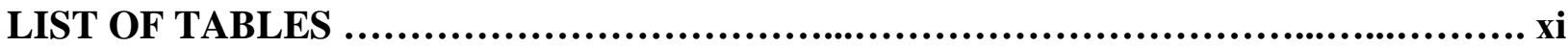

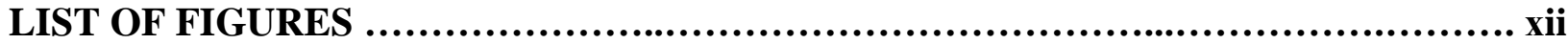

LIST OF ABBREVIATIONS .......................................................... xiv

CHAPTER 1: REVIEW OF THE LITERATURE ....................................... 1

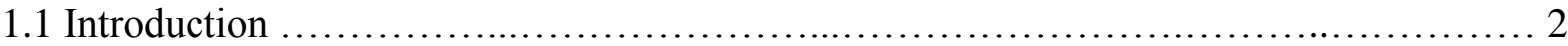

1.2 SCD1 Structure, Tissue Expression and Catalytic Reaction ............................... 4

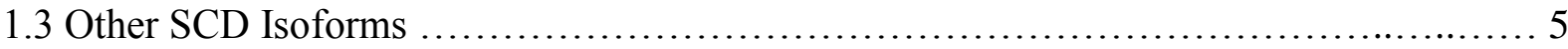

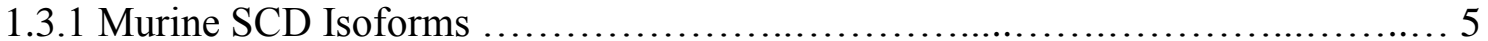

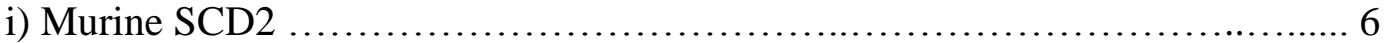

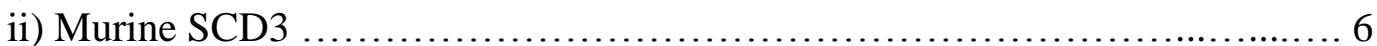

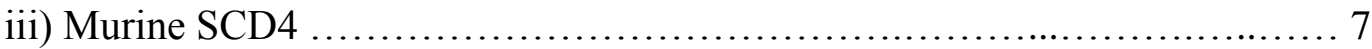

1.3.2 SCD Isoforms in Humans and Other Species ................................ 7

1.3.3 Substrate Specificity of SCD Enzymes ........................................ 8

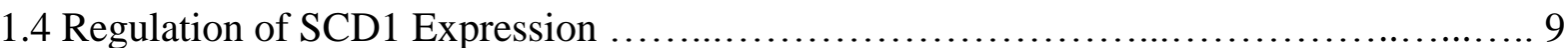

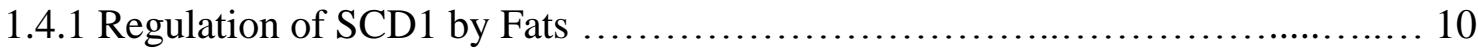

1.4.2 Regulation of SCD1 by Carbohydrates .................................. 12

1.4.3 Other Influences on SCD1 Expression or Activity ............................ 13

1.5 Metabolic Influences of SCD1 ..................................................... 15

1.5.1 The Influence of SCD1 on de novo Lipogenesis ............................. 16

1.5.2 The Influence of SCD1 on Adipocyte Differentiation and Cell Proliferation...... 19

1.5.3 The Influence of SCD1 on Fatty Acid Oxidation and Energy Expenditure ....... 20

1.5.4 The Influence of SCD1 on Inflammation ......................................... 22

1.5.5 Other Physiological and Metabolic Effects of SCD1 Activity ................... 24

1.6 SCD1 Activity at the Whole-Body, Adipose Tissue and Adipocyte Levels ............... 25

1.6.1 Estimated Whole-Body SCD1 Activity using Blood Fatty Acid Ratios .......... 25

1.6.2 General Overview of SCD1 in Adipose Tissue Models .......................... 27

i) SCD1 in Human Adipose Tissue ......................................... 29

ii) SCD1 in Rodent Adipose Tissue ........................................ 31

iii) SCD1 in Adipocyte Models ........................................... 33 


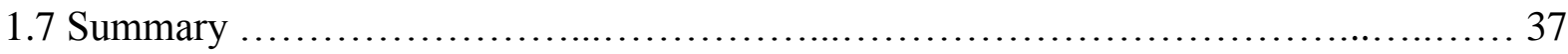

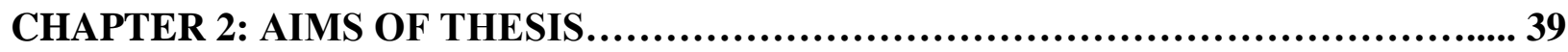

2.1 Rationale and Overall Objectives .................................................. 40

2.2 Specific Study Objectives and Hypotheses …................................... 41

\section{CHAPTER 3: INHIBITION OF STEAROYL-COA DESATURASE 1 IN DIFFERENTIATING 3T3-L1 PREADIPOCYTES UPREGULATES ELONGASE 6 AND DOWNREGULATES GENES AFFECTING TRIACYLGLYCEROL}

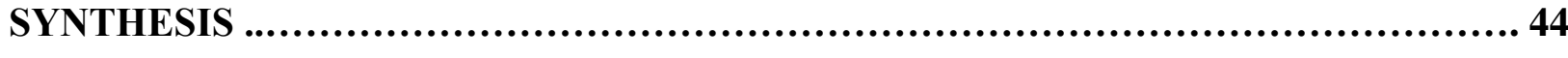

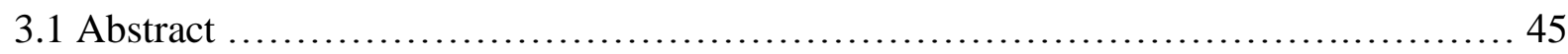

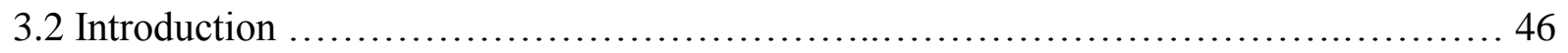

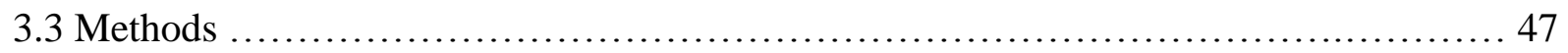

3.3.1 Chemicals and Cell Culture Reagents .................................... 47

3.3.2 Cell Culture Experiments ................................................. 48

3.3.3 Lipid Extraction and Quantification .......................................... 49

3.3.4 RNA and Protein Extraction ............................................... 50

3.3.5 Western Blot Analysis ......................................................... 51

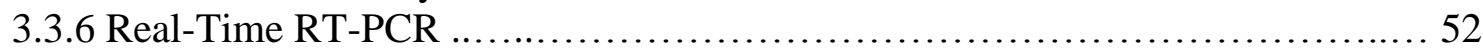

3.3.7 Microarray Analysis ..................................................... 52

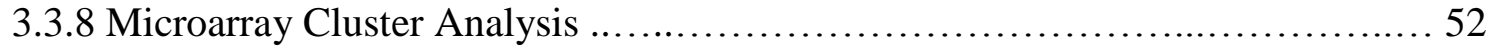

3.3.9 Statistical Analysis ......................................................... 53

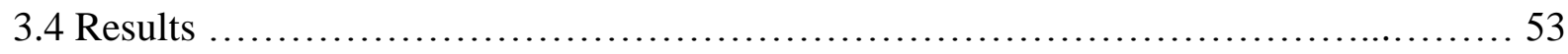

3.4.1 SCD1 Inhibition did not Influence Markers of Adipocyte Differentiation ....... 53

3.4.2 Effects of SCD1 Inhibition on Scd and Elovl6 Expression .................... 54

3.4.3 Effects of SCD1 Inhibition on FA Profiles in Differentiated Preadipocytes ..... 56

3.4.4 The Impact of SCD1 Inhibition on Biological Pathways in Differentiating

Preadipocytes . ......................................................... 58

3.4.5 Adipocyte TAG Abundance and SCD1 Inhibition ........................... 59

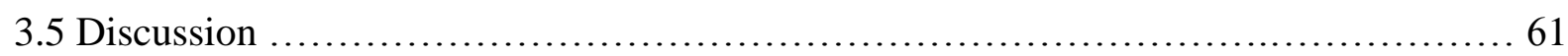

3.5.1 SCD $1_{\text {inhib }}$ Reduces SCD Activity but not Scd1 Gene Expression or Protein

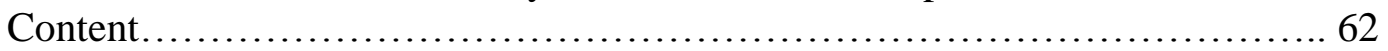

3.5.2 Potential Upregulation of ELOVL6 During SCD1 Inhibition to Prevent 16:0

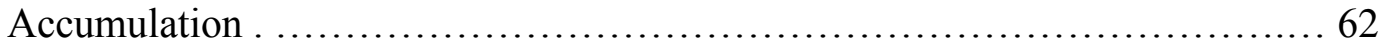

3.5.3 Altered MUFA Content in SCD1-Inhibited Adipocytes . ..................... 64

3.5.4 SCD1 Inhibition Alters Metabolic Pathways and Reduces TAG Synthesis ....... 64

3.5.5 Proposed Model for Reduced TAG Content in SCD1-Inhibited Adipocytes ...... 66

3.5.6 Summary and Perspectives ............................................. 68 


\section{CHAPTER 4: SCD1 INHIBITION DURING 3T3-L1 ADIPOCYTE DIFFERENTIATION REMODELS TRIACYLGLYCEROL, DIACYLGLYCEROL AND PHOSPHOLIPID FATTY ACID COMPOSITION ..................................70}

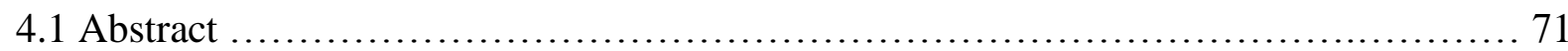

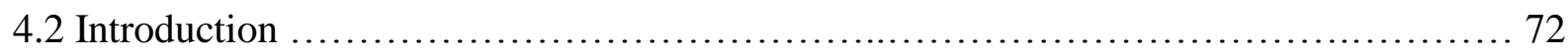

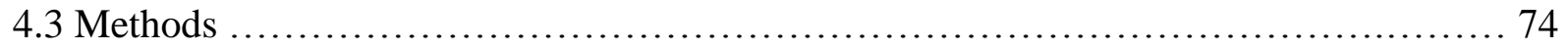

4.3.1 Chemicals and Cell Culture Reagents ........................................ 74

4.3.2 Cell Culture Experiments ..................................................... 74

4.3.3 Lipid Extraction and Quantification ......................................... 75

4.3.4 Gene Expression Analyses .................................................... 77

4.3.5 Western Blot Analyses .................................................... 77

4.3.6 Statistical Analyses ...................................................... 78

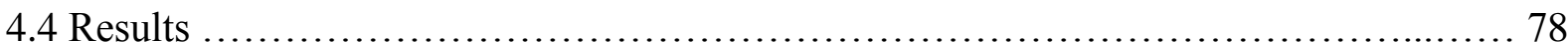

4.4.1 Effects of SCD1 Inhibition on Total Cellular Lipid Content Throughout Adipogenesis . ......................................................... 78

4.4.2 Effects of SCD1 Inhibition on Saturated Fatty Acids (SFAs) .................. 82

4.4.3 Effects of SCD1 Inhibition on Monounsaturated Fatty Acids (MUFAs) ......... 86

4.4.4 Effects of SCD1 Inhibition on Polyunsaturated Fatty Acids (PUFAs) ............. 86

4.4.5 SCD1 Inhibition Down-Regulates Genes Involved in PL Biosynthesis .......... 88

4.4.6 Effects of SCD1 Inhibition on Insulin Signalling and Cell Stress .............. 88

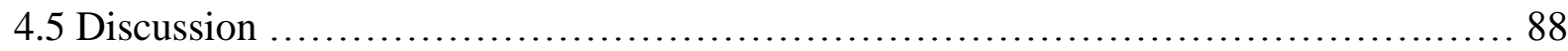

4.5.1 SCD1 Inhibition Alters Total Lipid Content within Lipid Fractions............... 90

4.5.2 Proposed Mechanism Contributing to Reduced PL and Increased Cellular

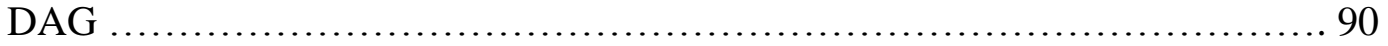

4.5.3 SCD1 Inhibition Alters SFA, MUFA and PUFA Content in Various Lipid

Fractions .............................................................. 93

4.5.4 SCD1 Inhibition Alters 18:0 Levels in Various Lipid Fractions ................... 93

4.5.5 SCD1 Inhibition Alters 16:1n-7 Levels in Adipocytes but not in Culture

Media .................................................................. 94

4.5.6 Summary and Perspectives ............................................ 96

CHAPTER 5: SCD1 MEDIATES THE INFLUENCE OF EXOGENOUS SATURATED AND MONOUNSATURATED FATTY ACIDS IN ADIPOCYTES: EFFECTS ON CELLULAR STRESS, INFLAMMATORY MARKERS, AND FATTY ACID ELONGATION ................................................................................... 97

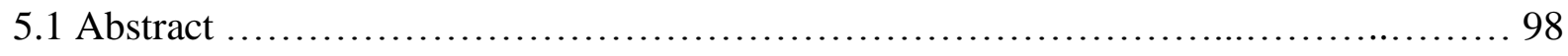

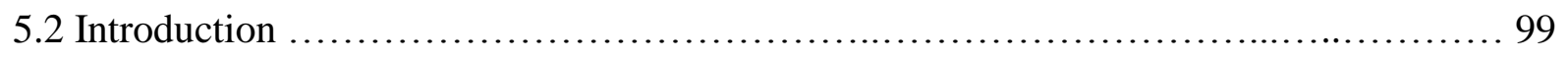




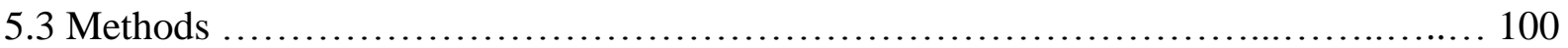

5.3.1 Chemicals and Cell Culture Reagents ..................................... 100

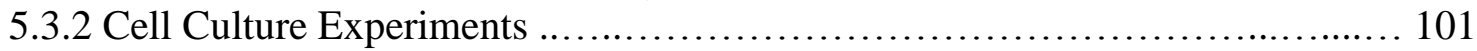

5.3.3 Lipid Extraction and Quantification ....................................... 102

5.3.4 ${ }^{13}$ PA Elongation Experiments................................................. 103

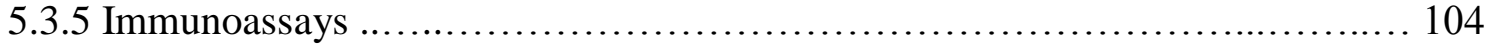

5.3.6 RNA and Protein Extraction .................................................. 104

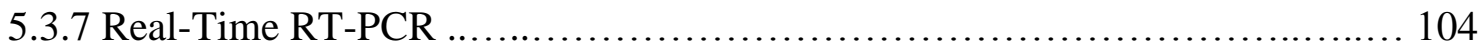

5.3.8 Western Blot Analyses .................................................... 105

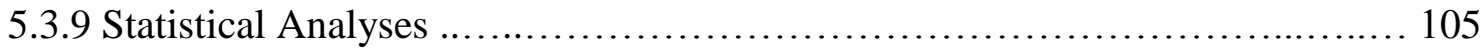

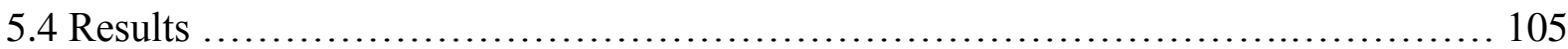

5.4.1 SCD1 Mediates the Effects of SFAs and MUFAs on Adipocyte FA

Metabolism .............................................................. 105

5.4.2 SFA and MUFA Treatments do not Influence Cellular Stress in SCD1-Inhibited

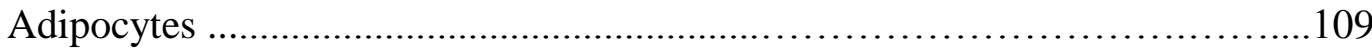

5.4.3 SCD1 Mediates SFA and MUFA Regulation of Cytokine Expression and

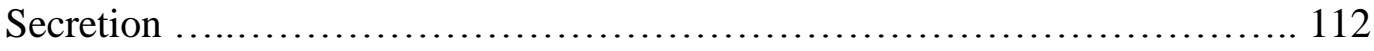

5.5 Discussion ...................................................................... 114

5.5.1 SCD1-Inhibited Adipocytes Effectively Take up Exogenous FAs, and UpRegulate PA Elongation to SA.......................................... 114

5.5.2 SCD1 Inhibition and Exogenous FAs do not Alter Markers of Cellular Stress . 115

5.5.3 The Effects of Exogenous FAs on Inflammatory Markers are Exacerbated in SCD1-Inhibited Adipocytes .......................................... 117

5.5.4 Summary and Perspectives ........................................... 118

CHAPTER 6: INTEGRATIVE DISCUSSION .......................................... 120

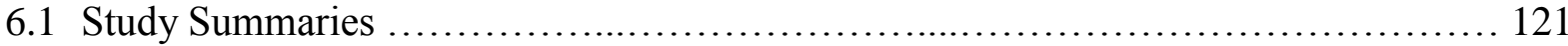

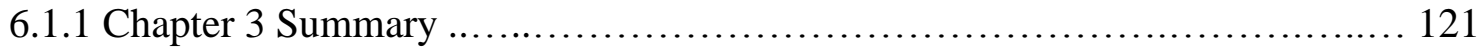

6.1.2 Chapter 4 Summary ........................................................ 122

6.1 .3 Chapter 5 Summary ..................................................... 122

6.2 SCD1 Activity has Protective Influences in Adipocytes .............................. 123

6.2.1 Ability to Tolerate Exogenous FAs ...................................... 123

6.2.2 The Prevention of DAG Accumulation and Impairments in Insulin Signaling .. 124

6.2.3 Production of a Potential Lipokine .......................................... 126

6.3 Altered Lipid Metabolism in SCD1-Inhibited Adipocytes .......................... 127

6.3.1 Altered Triacylglycerol Biosynthesis ................................... 128

6.3.2 Altered Phospholipid Fatty Acid Composition and the Maintenance of Oleate Levels ............................................................... 129

6.4 Prospective Research Possibilities and Remaining Questions ......................... 133 
6.4.1 FA Oxidation in States of Reduced SCD1 Activity ........................ 133

6.4 .2 Consideration of SCD2 ............................................ 134

6.4.3 Prospective Research Question - Why is SCD1 Activity Correlated with

Obesity? ..................................................... 135

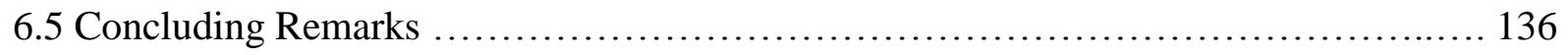

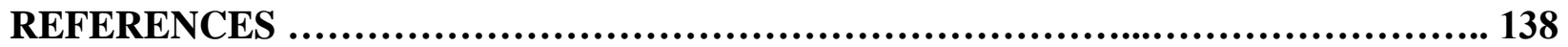




\section{$\underline{\text { List of Tables }}$}

\section{Chapter 4:}

Table 4.1 Total Fatty Acid Content of Lipid Fractions $\ldots \ldots \ldots \ldots \ldots \ldots \ldots \ldots \ldots \ldots \ldots \ldots \ldots$

Table 4.2 Total SFA, MUFA and PUFA Content of Lipid Fractions ................. 83

\section{Chapter 5:}

Table 5.1 Total Fatty Acid Content in Adipocytes ............................... 107 


\section{List of Figures}

\section{Chapter 1:}

Figure 1.1 The Endogenous Synthesis of MUFAs by SCD1 ...................... 3

\section{Chapter 1:}

Figure 1.2 SCD1-Derived MUFAs are the Preferred Substrates for TAGs

\section{Chapter 3:}

Figure 3.1 Ppary Gene Expression and Protein Content in Differentiating

Preadipocytes ............................................................ 54

Figure 3.2 Changes in Elovl6, $S c d 1$ and $S c d 2$ Gene Expression in Differentiating

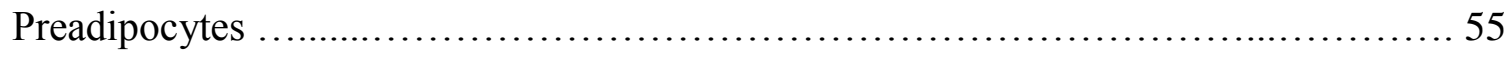

Figure 3.3 Changes in ELOVL6 and SCD1 Protein Expression in Differentiating

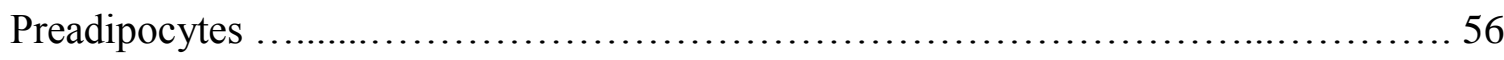

Figure 3.4 FA Abundance, Desaturation and Elongation Ratios in Differentiating

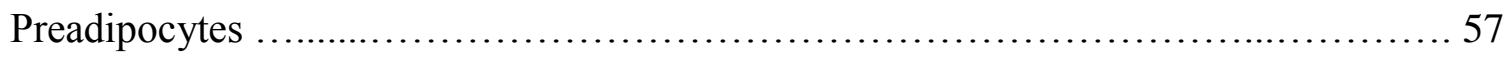

Figure 3.5 Biological Pathways Altered in Differentiating Preadipocytes .............. 60

Figure 3.6 Total TAG Content in Differentiating Preadipocytes with Control

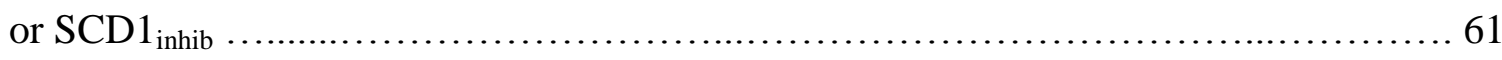

Figure 3.7 Proposed Mechanism by which SCD1 Inhibition Reduces Total TAG in

Differentiating Preadipocytes .

\section{Chapter 4:}

Figure 4.1 Relative Composition of Lipid Classes ................................. 81

Figure 4.2 Total 16:0 Abundance in Adipocytes ................................. 84

Figure 4.3 Total 18:0 Abundance in Adipocytes ................................ 85 
Figure 4.4 Total 16:1n-7 Abundance in Adipocytes ................................. 87

Figure 4.5 p-AKT and p-JNK Protein Content ...................................... 89

Figure 4.6 Proposed Mechanism Contributing to the Reduction in PL and Increase

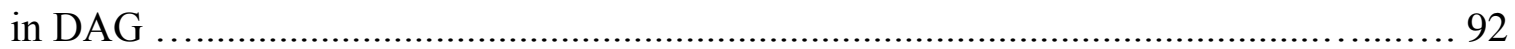

\section{Chapter 5:}

Figure 5.1 Relative ${ }^{13} \mathrm{SA}$ Content after $\mathrm{U}_{-}{ }^{13} \mathrm{C}$-labeled PA Treatment .................... 109

Figure 5.2 Markers of Cellular Stress in Adipocytes Treated with PA and PMA ...... 110

Figure 5.3 Markers of Cellular Stress in Adipocytes Treated with SA and OA ........ 111

Figure 5.4 Cytokines Secreted from Adipocytes Treated with FAs .................. 113

\section{Chapter 6:}

Figure 6.1 Integrated Effects of SCD1 Inhibition in Adipocytes .......................... 132 


\section{List of Abbreviations}

ACADVL

ACAT

ACC

ACOX

ADG

AGPAT

$\mathrm{AKO}$

AKT

AMPK

ANOVA

AT

BAT

$\mathrm{BF}_{3} \mathrm{MeOH}$

BMI

BSA

CE

C/EBP

CCL-5

CCR2

ChREBP

CLA very long chain acyl-CoA dehydrogenase

acyl-CoA cholesterol acyltransferase

acetyl CoA carboxylase

acyl-CoA oxidase

alkyl-2,3-diacylglycerol

acylglycerol-3-phosphate acyltransferase

adipose tissue-specific $S c d l$ knockout mouse

protein kinase B

adenosine monophosphate-activated protein kinase

analysis of variance

adipose tissue

brown adipose tissue

boron trifluoride-methanol

body mass index

bovine serum albumin

cholesteryl ester

CCAAT/enhancer binding protein

chemokine C-C motif ligand 5

CC-chemokine receptor 2

carbohydrate response-element binding protein

conjugated linoleic acid 
CPT

CRP

CVD

DAG

DGAT

Dex

DGK $\delta$

DHAP

DMEM

DMSO

DNA

ELOVL6

ER

ERK

FA

FABP4/ap2

FAS

FAT/CD36

FBS

FFA

FIAF/ANGPTL4

GC

GLUT carnitine palmitoyltransferase

C-reactive protein

cardiovascular disease

diacylglycerol

diacylglycerol acyltransferase

dexamethasone

diacylglycerol kinase delta

dihydroxyacetone phosphate

Dulbecco's modified eagle medium

dimethyl sulfoxide

deoxyribonucleic adid

elongase 6

endoplasmic reticulum

extracellular signal-regulated kinase

fatty acid

fatty acid binding protein 4/adipocyte protein 2

fatty acid synthase

fatty acid translocase/cluster of differentiation 36

fetal bovine serum

free fatty acid

fasting induced adipocyte factor

gas chromatography

glucose transporter 


\begin{tabular}{|c|c|}
\hline glycerol-3-P & glycerol-3-phosphate \\
\hline GPAT & glycerol-3-phosphate acyltransferase \\
\hline IBMX & 3-isobutyl-1-methylxanthine \\
\hline IL & interleukin \\
\hline JAK2 & janus kinase 2 \\
\hline JNK & jun $\mathrm{N}$-terminal kinase \\
\hline LAKO & combined adipose and liver $S c d l$ knockout mouse \\
\hline LDL & low-density lipoprotein \\
\hline LDLR & low-density lipoprotein receptor \\
\hline LKO & liver-specific $S c d l$ knockout mouse \\
\hline LPS & lipopolysaccharide \\
\hline LXR & liver $\mathrm{X}$ receptor \\
\hline MCP-1 & monocyte chemoattractant protein-1 \\
\hline mRNA & messenger ribonucleic acid \\
\hline mTOR & mammalian target of rapamycin \\
\hline MUFA & monounsaturated fatty acid \\
\hline NADH & nicotinamide adenine dinucleotide \\
\hline NF-1 & nuclear factor 1 \\
\hline $\mathrm{NF}-\kappa \beta$ & nuclear factor kappa-light-chain-enhancer of \\
\hline & activated $\beta$ cells \\
\hline $\mathrm{OA}$ & oleate/oleic acid \\
\hline $\mathrm{Ob}$ & leptin/obese gene \\
\hline p38 MAPK & p38 mitogen-activated protein kinase \\
\hline
\end{tabular}


PA

PBS

PCK/PEPCK

Pen-strep

PFK

PGC1 $\alpha$

PGMM

PI3K

PL

PMA

PPAR

PTA

PUFA

RA

RPLP0

SA

SCD1

SCD1-/-

$\mathrm{SCD} 1_{\text {inhib }}$

SEM

SFA

siRNA

SKO palmitate/palmitic acid

phosphate buffered saline

phosphoenolpyruvate carboxykinase

penicillin-streptomycin

phosphofructokinase

PPAR co-activator $1 \alpha$

parsimonious Gaussian mixture models

phosphoinositide 3-kinase

phospholipid

palmitoleate/palmitoleic acid

Peroxisome proliferator-activated receptor

phosphatidic acid

polyunsaturated fatty acid

retinoid acid

ribosomal protein, $60 \mathrm{~S}$

stearate/stearic acid

stearoyl-CoA desaturase 1

Scdl knockout mouse

SCD1 inhibitor

standard error of the mean

saturated fatty acid

small inhibitory ribonucleic acid

skin-specific $S c d 1$ knockout mouse 
SNP

SREBP

STAT

$\mathrm{T} 2 \mathrm{D}$

TAG

TBST

TLC

TLR4

TNF $\alpha$

UCP

VA

VCAM-1

VLDL

WAT single nucleotide polymorphism

sterol regulatory element binding protein

signal transducer and activator of transcription

type 2 diabetes

triacylglycerol

tris-buffered saline- $0.1 \%$ Tween-20

thin layer chromatography

toll-like receptor 4

tumour necrosis factor alpha

uncoupling protein

vaccenate/vaccenic acid

vascular cell adhesion molecule 1

very-low density lipoproteins

white adipose tissue 


\section{CHAPTER 1:}

REVIEW OF THE LITERATURE 


\section{$\underline{1.1 \text { Introduction }}$}

The obesity epidemic has risen to global proportions in recent years. In 2014, over 1.9 billion individuals worldwide were classified as overweight, and 600 million people were classified as obese [1]. Obesity coincides with a myriad of health complications including type 2 diabetes, hypertension, cancer, and cardiovascular disease [2]. The expansion of adipose tissue (AT) that occurs with obesity, also contributes to the chronic low-grade inflammatory state that often defines the obese phenotype [3,4]. During AT expansion, preadipocytes are differentiated into mature adipocytes that contain lipid droplets filled primarily with triacylglycerol (TAG) [5, 6]. This process, referred to as adipogenesis, occurs alongside coordinated changes in gene expression and protein content that promote the biosynthesis of fatty acids (FAs) and lipids (e.g., TAGs, cholesteryl esters (CEs) and phospholipids (PLs)) [5, 7]. Stearoyl-CoA desaturase 1 (SCD1) is among this panel of genes, and therefore plays a critical role in adipocyte lipid metabolism. Given that SCD1 is involved in adipogenesis and AT expansion, it is perhaps not surprising that SCD1 activity is positively associated with obesity in humans [8]. SCD1 is therefore a very important target in adipocyte and obesity-related research.

SCD1 is a delta-9 desaturase enzyme responsible for the conversion of saturated fatty acids (SFAs) to monounsaturated fatty acids (MUFAs) [9]. Specifically, SCD1 preferentially converts dietary and de novo synthesized palmitate (PA; 16:0) and stearate (SA; 18:0) to palmitoleate (PMA; 16:1n-7) and oleate (OA; 18:1n-9), respectively [10] (Figure 1.1). PMA and OA are the most abundant MUFAs in living organisms and are used in the synthesis of lipid species including PLs, TAGs and CEs $[10,11]$. In addition, MUFAs produced by SCD1 are known to mediate lipid-activated signal transduction (e.g., synergizing the activation of protein 
kinase $\mathrm{C}$ by DAGs) and act as possible inter-organ signaling molecules (i.e., 'lipokines') [12-14]. By influencing MUFA content, SCD1 has the potential to impact signaling processes as well as the synthesis of PLs, TAGs and CEs, which in turn can modify whole-body metabolism.

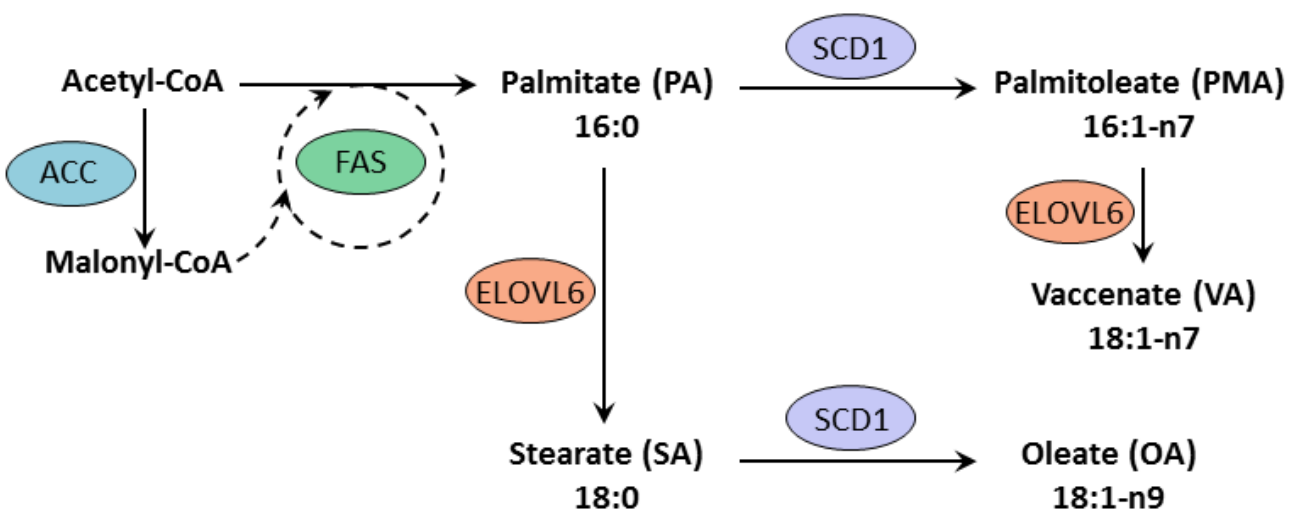

Figure 1.1: The endogenous synthesis of MUFAs by SCD1. ACC, acetyl-CoA carboxylase; FAS, fatty acid synthase; ELOVL6, elongase 6; SCD1, stearoyl-CoA desaturase 1.

A reduction in $S c d 1$ gene expression, as seen in $S c d 1$-/- mice, was shown to provide protection from diet-induced obesity and ensuing metabolic complications. For instance, Scd1-/mice had reduced whole body adiposity, reduced cellular lipid accumulation, increased energy expenditure, increased FA oxidation, and reduced insulin resistance [11, 15, 16]. However, SCD1 deficiency (i.e., in $S c d 1-/-$ mice or cellular models) may also cause $\beta$-cell dysfunction, skin alopecia, and certain inflammatory diseases such as atherosclerosis and intestinal colitis [17-20]. To date, the majority of SCD1 research has often examined metabolism in the liver, primarily because the liver is the principle site for de novo lipogenesis. In addition, $S c d 1$ expression can be significantly regulated in the liver by dietary factors (e.g., a high carbohydrate diet) [21, 22], which has contributed to the liver becoming a focal point for SCD1-related research [23-26]. However, $S c d 1$ is expressed in all murine lipogenic tissues [21], and its high expression in AT 
suggests that the $S c d l$ isoform may play an equally important role in AT metabolism. This is supported by findings in AT-specific Scd1-/- (AKO) mice, where adiponectin expression was reduced and gene expression of pro-inflammatory cytokines was increased [27]. However, our understanding of how reduced $S c d l$ expression and activity can influence AT and adipocyte metabolism is currently incomplete. For instance, it remains uncertain to what extent an adipocyte-specific reduction in SCD1 activity can impact lipid metabolism, inflammation and insulin sensitivity.

In addition to describing SCD1 structure, tissue expression and catalytic function, the forthcoming sections will outline the existing evidence regarding the involvement of SCD1 in biological processes including lipid biosynthesis, cell proliferation, inflammation, and FA oxidation. The majority of our knowledge regarding SCD1 stems from work that has examined the liver, and will therefore be reviewed; however, findings from AT and adipocytes will be discussed wherever possible and will remain the focus of later subsections.

\subsection{SCD1 Structure, Tissue Expression and Catalytic Reaction}

SCD1 is a $37-\mathrm{kDa}$ membrane bound enzyme located on the endoplasmic reticulum (ER), and contains four trans-membrane domains [10]. The $\mathrm{NH}_{2}$ and $\mathrm{COOH}$ terminals, as well as the eight conserved histidine residues that comprise the His box (i.e. where iron is bound in the catalytic centre of the enzyme), are all positioned towards the cytosol [10]. In mammals, Scdl expression is ubiquitous $[18,28]$, but is notably high in the liver, white AT (WAT), brown AT (BAT) [10] and in secretory glands (i.e., meibomium [29], Hardarian [30] and prepeutial glands [31]). 
The aerobic desaturation reaction catalyzed by SCD1 entails the introduction of a cisdouble bond into FA substrates at the delta-9,10 position $[18,32]$. Specifically, the oxidative reaction involves a multi-component enzyme system containing the flavoprotein NADHdependent cytochrome b5 reductase, an electron acceptor (cytochrome b5), reducing equivalents $(\mathrm{NADH})$, and the SCD1 protein $[9,33]$. Electrons are passed from $\mathrm{NADH}$ to cytochrome b5 via NADH-dependent cytochrome b5 reductase, followed by transfer to SCD1 and then to $\mathrm{O}_{2}$, where $\mathrm{O}_{2}$ is ultimately reduced to $\mathrm{H}_{2} \mathrm{O}[10,33]$.

\subsection{Other SCD Isoforms}

SCD1 is the predominant enzyme isoform in lipogenic tissues of mice and humans; however, it is important to acknowledge that other SCD isoforms also exist and that they have identical desaturation functions to SCD1. Interestingly, the tissue distribution and substrate specificity for each SCD isoform varies, which may suggest that each isoform has a distinct role in metabolism despite having an identical catalytic function to SCD1. In total, four murine SCD isoforms and two human SCD isoforms have been identified over the past 30 years.

\subsubsection{Murine SCD Isoforms}

Mice, which are often used to generate models of SCD-deficiency for research purposes [9], express four SCD isoforms (SCD1-4) that are all located on chromosome 19 within a 200 kilobase pair region $[18,22,28,34,35]$. The murine $S c d$ genes code for proteins 350-360 amino acids in length, and have more than $80 \%$ sequence similarity [18]; however, small variations in the 5'-flanking region of the genes result in varying tissue expression of each isoform. 


\section{i) Murine SCD2}

Murine $S c d 2$ is similar to the $S c d 1$ isoform in that it is ubiquitously expressed and shares a high sequence similarity [35]. $S c d 2$ expression is highest in the brain and has been proposed to have a role in the early development of neonates [36]. The SCD2 protein is 358 amino acids in length and has more than $87 \%$ sequence similarity to SCD1 [35]. As well as its presence in the brain, $S c d 2$ has been detected in AT [28], skin [36], the Harderian gland [30], and the preputial gland [31]. The induction of $S c d 2$ expression by a high-carbohydrate diet has also been noted in the kidney, heart, lungs and spleen [10, 22]. Whole body $S c d 2$ deficiency is not viable and $S c d 2-/-$ neonatal mice generally do not survive more than 24 hours $[28,36]$. The fatal effect of early-life $S c d 2$-deficiency is likely due to defects in epidermal permeability barriers, which results in severe dehydration [28]. Scd2-/- mice that survive into adulthood have healthy sebaceous glands and hair growth. Interestingly, hepatic $S c d 2$ expression is important for TAG production in embryos and neonates, but $S c d 2$ is very lowly expressed in the liver of adult mice when $S c d 1$ becomes the dominant isoform [36].

\section{ii) Murine SCD3}

SCD3 is a protein 359 amino acids in length and its sequence similarity is $88.7 \%$ to SCD1 [22]. Scd3 is primarily expressed in the sebaceous glands of the skin [22], but is also found in the Harderian and prepeutial glands [31]; however, $S c d 3$ expression in these tissues is generally lower than $S c d 1$ expression. In the skin, $S c d 3$ is often found in fully differentiated sebocytes, while $S c d 1$ is generally confined to undifferentiated sebocytes [10]. The fact that $S c d 3$ is predominantly detected in the skin suggests that it may play an important role in skin biology. 
iii) Murine SCD4

The SCD4 isoform is a 352 amino acid protein and its sequence similarity with $S c d 1$ is $79 \%$ [21]. SCD4 is exclusively detected in the heart [21]. Although the functional importance of the SCD4 isoform is not fully known, Miyazaki et al. have shown that a high carbohydrate fatfree diet can induce $S c d 4$ expression [21]. $S c d 4$ was also elevated in the hearts of leptin-deficient $o b / o b$ mice [21], and could therefore be partially responsible for the development of the 'fatty heart' observed in these mice. Although the purpose of having more than one SCD isoform is currently unknown, previous work has suggested that this could be due to the different tissue distribution and expression patterns of each SCD isoform, or due to differential regulation by dietary factors $[10,32]$.

\subsubsection{SCD Isoforms in Humans and Other Species}

Humans express only two SCD enzyme isoforms throughout the body: SCD1 and SCD5 [37-39]. Prior research has indicated that the two human $S C D$ transcripts originate from one structural $S C D$ gene via alternative polyadenylation sites [40]. The human SCD1 isoform, which is located on chromosome 10 , is highly homologous $(\sim 85 \%)$ with murine $S c d 1$ and is predominantly expressed in AT and liver [28]; however SCD1 expression has also been noted in skeletal muscle, heart, brain, lungs and pancreas [38, 39, 41]. In contrast to SCD1 expression, SCD5 is mostly confined to the pancreas and brain [37,42], yet low SCD5 expression has also been detected in the lungs, kidneys, heart and placenta [37, 39, 43]. SCD5, which is unique to primate species, is located on chromosome 4 and its amino acid sequence is fairly dissimilar to any of the murine $S c d$ isoforms [37, 42]. Finally, while other human $S C D$ isoforms have not been 
identified, previous work has shown that a $S C D$ pseudogene exists on chromosome 17; however, this gene is transcriptionally inactive $[40,44]$.

SCD isoforms have also been identified in numerous other species including the rat (which express the $S c d 1$ and $S c d 2$ isoforms [45, 46]), orangutan [37], chimpanzee [37], sheep [47], hamster [48], pig [37], cow [37], goat [37], yeast [49], Drosophila [10, 50], Mortierella alpine [51], and C. elegans [52]. Similarities in the SCD orthologues from different species include a high degree of sequence similarity and conserved catalytic activity of the encoded desaturase enzymes [38]. In contrast, key SCD differences across species include the number of isoforms and their respective tissue distributions, as well as the regulatory mechanisms involved in producing $S c d$ mRNA transcripts $[38,40]$.

\subsubsection{Substrate Specificity of SCD Enzymes}

While the primary catalytic desaturation reaction is conserved across the SCD isoforms, substrate specificity can differ [53]. The structure of the SCD catalytic unit enables the binding of FA substrates anywhere from 9-20 carbons in length, where 12-19 carbon FAs are preferentially desaturated $[9,18,54]$. Importantly, the high abundance of PA and SA cause these FAs to be the predominant substrates of SCDs [9]. Murine SCD1, 2 and 4 desaturate PA and SA, but show preference towards SA $[28,55]$. However, the substrate specificity assays used to determine these preferences cannot definitively discount that SA desaturation efficiency, and not preference, could underlie these observations. In contrast to SCD1, 2 and 4, murine SCD3 almost exclusively desaturates PA and not SA [28, 55].

In addition to isoform-specific substrate specificities, tissue-specific substrate specificity

has also been shown. Hepatic microsomal SCDs in rats have been suggested to prefer SA as a 
substrate over PA, and then to an even lesser extent myristate (14:0), and then pentadecanoate (15:0) [54]. Kim et al. proposed that SCD1 in murine 3T3-L1 adipocytes can desaturate either PA or SA depending on the abundance of each FA within the cells [53]. Furthermore, they noted that SCD2 might be selective towards SA desaturation compared to PA [53]. Since Scd2 expression is often higher in undifferentiated 3T3-L1 cells, Kim et al. noted that the SCD2 enzyme may be responsible for the majority of FA desaturation prior to differentiation [53]. Yee et al. have additionally hypothesized that hepatocytes may 'compartmentalize' SCD1 activity in subcellular locations according to the origin of the FA substrate (i.e., dietary or de novo lipogenesis) [56]. While interesting, this hypothesis was primarily proposed based on the differential effects of SCD1 inhibitors and disparities of desaturation indices (i.e., ratios comparing endogenous vs. exogenous FA sources, or ratios comparing labeled PA vs. labeled SA treatments) [56]. Therefore, additional investigations co-treating cells with differentially labeled PA and SA, as well as unlabeled FAs, are needed to confirm this hypothesis. Finally, in vivo work using human plasma has estimated a SCD conversion rate of $14 \%$ for SA to OA; however, the conversion rate for PA to PMA was only 2\% [57]. Collectively, it appears that while SA is often denoted as the dominant SCD substrate, the availability of FAs will ultimately influence desaturation specificity.

\subsection{Regulation of SCD1 Expression}

SCD1 expression in humans and mice, as well as SCD1 activity, can be regulated by factors that include dietary nutrients, hormones (e.g., insulin, estrogen and leptin), and protein degradation (e.g., proteases) [58]. In general, the regulation of $S C D 1$ expression is facilitated by 
numerous transcription factors and co-activators that have been shown to bind to the promoter region of murine and human SCD1 [58]. These transcription factors and co-activators include sterol regulatory element binding protein 1c (SREBP-1c), liver X receptor (LXR), peroxisome proliferator-activated receptor $\alpha(\mathrm{PPAR} \alpha), \mathrm{CCAAT} /$ enhancer binding protein $\alpha(\mathrm{C} / \mathrm{EBP} \alpha)$ and PPAR co-activator $1 \alpha(\mathrm{PGC} 1 \alpha)$, among others $[58,59]$. The following subsections will briefly discuss how murine $S c d 1$ (and in certain cases, human $S C D 1$ as well as SCD1 activity) can be regulated by fats, carbohydrates, and other factors including hormones and protein degradation.

\subsubsection{Regulation of SCD1 by Fats}

Although the differential effects of specific dietary fats (e.g., specific SFAs) on SCD1 expression are not fully established, several studies have shown that dietary fats can influence SCD1 expression in several metabolic tissues of humans and rodents. In human adults, a high fat diet (40\% total energy from fat; $22 \mathrm{~g} / 100 \mathrm{~g}$ as PA) has been shown to increase estimated SCD1 activity in the liver (estimated using FA product-to-precursor ratios in plasma); however, the effects of the high fat diet on estimated SCD1 activity were not as robust as those of a high carbohydrate diet (75\% total energy from carbohydrates) [60]. Additionally, SCD1 expression in AT was only increased by the high carbohydrate diet and not the high fat diet [60], which suggests that dietary fats do not induce $S C D 1$ expression in AT as strongly as carbohydrates. A high fat diet (55\% calories from fat) was also able to increase hepatic $S c d 1$ expression in obesity prone C57B1/B6 mice [61]. One of the few studies that has examined the influence of specific SFAs on $S c d 1$ showed that dietary SA induced hepatic Scdl expression and SCD1 protein levels in mice, which occurred prior to the up-regulation of lipogenic genes in the liver (i.e., acetylCoA carboxylase (Acc), and fatty acid synthase (Fas)) [62]. However, this same study observed 
that dietary SA was unable to induce hepatic lipogenic gene expression in Scd1-/- mice [62]. This may imply that SA induction of lipogenesis requires the activation of $S c d l$, and that the catalytic function of SCD1 (i.e., the production of OA from SA) is required to induce lipogenic gene expression.

The activation of $S c d 1$ transcription by SFAs may partly stem from the ability of SFAs to induce the expression of transcription factors such as SREBP-1c and PGC1 $\beta$ [58, 63]. PPARs have also been implicated in FA activation of $S c d 1$ [58], where SFAs such as SA can up-regulate genes related to PPAR signalling as well as the expression of Ppary in adipocytes [64]. PPAR $\gamma$ plays an important role in activating $S c d 1$ in AT and adipocytes $[65,66]$; however, treating 3T3L1 adipocytes with troglitazone or pioglitazone (PPAR $\gamma$ agonists) appeared to reduce $S c d 1$ expression [53, 67]. Hepatic $S c d 1$ expression in mice is also induced by PPAR $\alpha$ agonists (e.g., fenofibrate, a drug used to reduce cholesterol levels) [68]. Evidence that SFAs can influence $S C D 1$ in other tissues or cell types include a demonstration in human myotubes, where SCD1 expression was increased by PA [69].

The ability for unsaturated FAs to regulate $S c d l$ expression and activity in AT or adipocytes has not been extensively examined. One study suggested that the ability of polyunsaturated FAs (PUFAs) to repress lipogenic gene expression in AT may involve the production of PUFA derivatives such as eicosanoids [70], although work in 3T3-L1 adipocytes counters this notion [71]. Work by Sessler and Ntambi showed that treating 3T3-L1 adipocytes with n-6 FAs (arachidonate and linoleate) or n-3 FAs (eicosapentaenoate) decreased Scd1 mRNA content [71]. This aligned with previous reports in the liver, which have shown that both $n-3$ and n-6 PUFAs can strongly inhibit hepatic lipogenic genes including $S c d 1$ [72]. The repression of hepatic Scdl expression by PUFAs partially involves the inhibition of SREBP-1c maturation 
(i.e., proteolytic cleavage of precursor SREBP and the translocation of mature/active SREBP to the nucleus) [73]. Given the potential for $S c d l$ to influence lipogenesis, future studies could consider examining if exogenous PUFAs can down-regulate lipogenic pathways in hepatocytes and adipocytes through a reduction in $S c d l$ expression, and should determine if distinct PUFAs can differentially affect this process.

\subsubsection{Regulation of SCD1 by Carbohydrates}

One of the most well characterized regulators of $S c d 1$ expression is carbohydrates. Dietary studies in mice have shown on several occasions that an ad libitum high carbohydrate diet (e.g. 55-60\% by weight) can substantially induce hepatic $S c d 1$ expression [10, 16, 74]. Activation of hepatic Scdl by carbohydrates primarily involves SREBP-1c, carbohydrate response-element binding protein (ChREBP), and nuclear factor 1 (NF-1) [10, 16, 58, 75]. Hepatic Scdl can also be up-regulated by insulin through phosphoinositide 3-kinase (PI3K), protein kinase $\mathrm{B}(\mathrm{AKT})$, and mammalian target of rapamycin (mTOR) dependent signaling pathways $[58,76]$. Specifically, the PI3K, AKT and mTOR dependent signaling pathways can target insulin response elements in the $S c d 1$ promoter, thereby up-regulating hepatic $S c d 1$ expression [76]. Studies examining the effects of specific carbohydrates on $S c d 1$ have found that sucrose [77], glucose [26] and fructose [26, 78, 79] can all positively regulate hepatic Scdl expression and SCD1 activity. Furthermore, the lipogenic effects of carbohydrates do not appear to occur in states of SCD1-deficiency, as evidenced by the protection of Scd1-/- mice from obesity when fed a high carbohydrate diet $[9,26,78]$. This further implicates the involvement of Scdl in the induction of lipogenesis by carbohydrates. 
In contrast to the liver, the effects of a high sucrose, glucose or fructose diet on Scd1 expression in AT have not been fully unraveled. The few studies that have examined carbohydrate-related regulation of $S c d 1$ in AT or adipocytes have shown that carbohydrates can stimulate $S c d 1$ expression in 3T3-L1 adipocytes through the induction of insulin and SREBP-1c $[58,80,81]$. Other studies in 3T3-L1 preadipocytes have also shown that C/EBP and NF-1 act as transcriptional activators of $S c d 1[59,82]$. In addition, the binding of NF-1 to the $S c d 1$ promoter may facilitate 3T3-L1 adipocyte differentiation [82]. Future work could consider using 3T3-L1 adipocytes to test if common carbohydrates (e.g., glucose, sucrose and fructose) can increase the expression and activity of SCD1 through C/EBP, NF-1, or SREBP-1c dependent mechanisms.

\subsubsection{Other Influences on SCD1 Expression or Activity}

The ability for hormones including leptin, estrogen and thyroid hormone to regulate $S c d 1$ expression and activity has been reviewed in detail [28, 58, 83]. Briefly, leptin has inhibitory effects on $S c d l$, as demonstrated by the ability of leptin injections to decrease hepatic and AT $S c d 1$ expression in $o b / o b$ mice [84], as well as hepatic $S c d 1$ expression and protein content in control mice [85]. Work in HepG2 cells has shown that leptin targets the leptin response element of the $S C D 1$ promoter via signalling pathways that involve extracellular signal-regulated kinases (ERK1/2) as well as janus kinase 2 (JAK2) [86]. Leptin may blunt the activation of SCD1 by insulin by inhibiting insulin signalling pathways (e.g., tyrosine phosphorylation of insulin receptor substrates) or by reducing SCD1 protein stability [58].

Estrogen generally antagonizes $S c d l$ expression, as evidenced by the ability of estrogen administration to reduce human AT SCDI expression [87] and by work showing that estrogen therapy decreases AT $S c d l$ expression in high-fat fed mice [88]. The ability of estrogen to reduce 
Scdl expression in AT has been hypothesized to involve a reduction in Srebp-1c expression [88]. In the liver, estrogen treatment was also shown to reduce hepatic Scdl expression [88]. Additionally, estrogen receptor knockout mice have higher hepatic $S c d l$ expression compared to wild type mice [89]. The ability of estrogen to decrease $S c d l$ expression in the liver, unlike AT, does not appear to involve Srebp-1c, but could entail the estrogen-targeted up-regulation of Stat3 and the subsequent reduction of hepatic lipogenic gene expression $[89,90]$.

Other factors that influence $S c d l$ expression include thyroid hormone, which has been shown to repress $S c d l$ expression. Specifically, the administration of triiodothyronine $\left(\mathrm{T}_{3}\right.$, the active form of thyroid hormone) to hypothyroid mice significantly reduced hepatic Scdl expression [91]. A similar repressive effect of $\mathrm{T}_{3}$ on $S C D 1$ expression was also seen in HepG2 cells via a thyroid hormone-responsive binding site on the SCD1 gene [91]. Retinoic acid (RA) has also been demonstrated to inhibit Scdl expression in 3T3-L1 preadipocytes in early differentiation, but increase $S c d 1$ expression at later time points of differentiation [92]. More detailed work has been conducted in the liver, where RA has been shown to induce $S c d l$ expression through transcriptional regulation by the RA receptor and its heterodimerization with the retinoid $\mathrm{X}$ receptor [93]. A diet high in cholesterol has also been shown to induce hepatic Scdl expression in rats, but does not appear to have this influence in AT [94, 95]. Additional

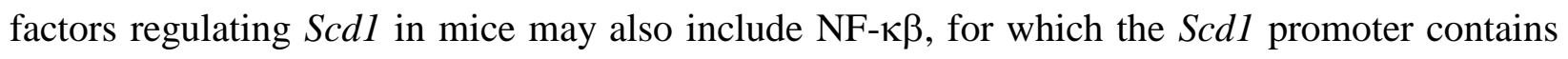

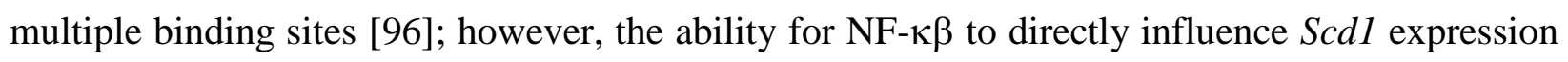
has not been directly observed. Future studies could test this hypothesis by stimulating NF- $\kappa \beta$ in cell culture models (e.g., 3T3-L1 adipocytes) with NF-к $\beta$ agonists (e.g., betulinic acid) and examining the impact on $S c d l$ expression. 
Finally, regulation of SCD1 can also occur at the protein level. An examination of rat liver demonstrated that the half-life of SCD1 protein is only 2-4 hours [28, 97]. Therefore, any factors that influence the stability or degradation of the SCD1 protein have the potential to alter the level of SCD1 activity [33, 39]. For example, ATPase p97 is involved in the regulation of proteasome-dependent degradation of SCD1 in several cultured cell lines (e.g., 3T3-L1 cells, Chinese hamster ovary-K1 cells, and HeLa cells) [98], and therefore regulates overall cellular SCD1 activity. Interestingly, although ATPase p97 expression is similar in the AT of obese and control adults, it is more highly expressed in visceral AT as compared to subcutaneous AT [99]. This may suggest that AT SCD1 regulation by proteases is depot-specific in humans.

\section{$\underline{1.5 \text { Metabolic Influences of SCD1 }}$}

SCD1 can influence numerous metabolic processes that ultimately influence cell, tissue and whole-body health. For instance, SCD1 plays an important part in promoting hepatic de novo lipogenesis and in synthesizing FAs for the production of essential lipid species (e.g., TAGs, PLs

or CEs) [16]. In murine models, $S c d l$ expression and activity have also been implicated in cellular proliferation and adipocyte differentiation, which has a significant influence on adiposity [7, 66]. Moreover, SCD1 activity has been demonstrated in rodent models to influence FA oxidation [100, 101], skin health [29, 102], membrane fluidity [66] and inflammation [18, 27]. Collectively, SCD1 can be considered a central regulator of whole body metabolism which makes it physiologically significant and important to investigate in health research. 


\subsubsection{The Influence of SCD1 on de novo Lipogenesis}

A well characterized trait of $S c d 1$ /- mice is their protection from weight gain on a high carbohydrate diet [15]. To date, many of the investigations examining Scd1-/- mice have hypothesized that the protection from diet-induced obesity stems from a reduction in hepatic lipogenic gene expression $[15,62]$. Furthermore, since $S c d 1$ is the predominant isoform in most lipogenic tissues $[28,36]$, this may partly explain why $S c d 1-/-$ mice have reduced whole body lipid levels in addition to a decrease in FA synthesis [62]. The expression of hepatic genes involved in FA-synthesis including Fas, Acc, Srebp-1, and Elovl6 is altered in Scd1-/- mice [15, 16]. For instance, while a high fructose diet normally induces hepatic Scdl prior to the subsequent up-regulation of hepatic Acc, Fas and Srebp-1 expression, this induction does not occur in Scd1-/- mice [26]. Supplementing the high fructose diet with $20 \%$ (by weight) OA (i.e., a product of SCD1) partially restores the induction of hepatic Srebp-1 expression in Scd1-/mice; however, the levels of Srebp-1 expression in Scd1-/- mice fed the OA diet did not reach the levels seen in control mice [26]. Miyazaki et al. attributed these findings to the necessity of endogenously synthesized OA (i.e., via SCD1) to achieve full induction of Srebp-1 expression by a fructose diet [26]. While this hypothesis has merit, additional experiments should confirm whether endogenously-synthesized OA can more strongly promote lipogenesis in hepatocytes or adipocytes compared to dietary/exogenous OA. Building on these findings, Sampath et al. suggested that endogenously-synthesized OA (i.e., via SCD) can act as a signal of energy flux, which in turn can induce FA synthesis and lipogenesis [62]. This is also an interesting notion that could be further investigated using global profiling techniques (e.g., transcriptomics and metabolomics) in an attempt to determine the global mechanisms allowing OA to act as a signalling molecule. 
In addition to suppressing the expression of genes for FA synthesis, SCD1-deficiency is also associated with a reduction in the production and content of various lipid species (i.e., TAGs, CEs, PLs, wax esters, and alkyl-diacylglycerols) in tissues such as the liver, AT and eyelids [29, 30]. There are two major lines of thinking regarding how SCD1-deficiency contributes to the reduction in lipid biosynthesis and content. Firstly, MUFAs produced by SCD1 are the primary substrates incorporated into larger lipid molecules [16]. For example, PMA and OA are the predominant FAs found in TAGs, CEs and PLs of the liver and AT (Figure 1.2) [11, 16], and OA is the main substrate used to produce 1-alkyl-2,3-diacylglycerol for the Harderian gland [30]. Moreover, Miyazaki et al. have proposed that endogenously synthesized MUFAs (via SCD1), are more critical substrates for hepatic TAG biosynthesis when compared to dietary MUFAs [16]. SCD1 therefore has an important role producing the MUFAs that are necessary for the biosynthesis of these lipids.

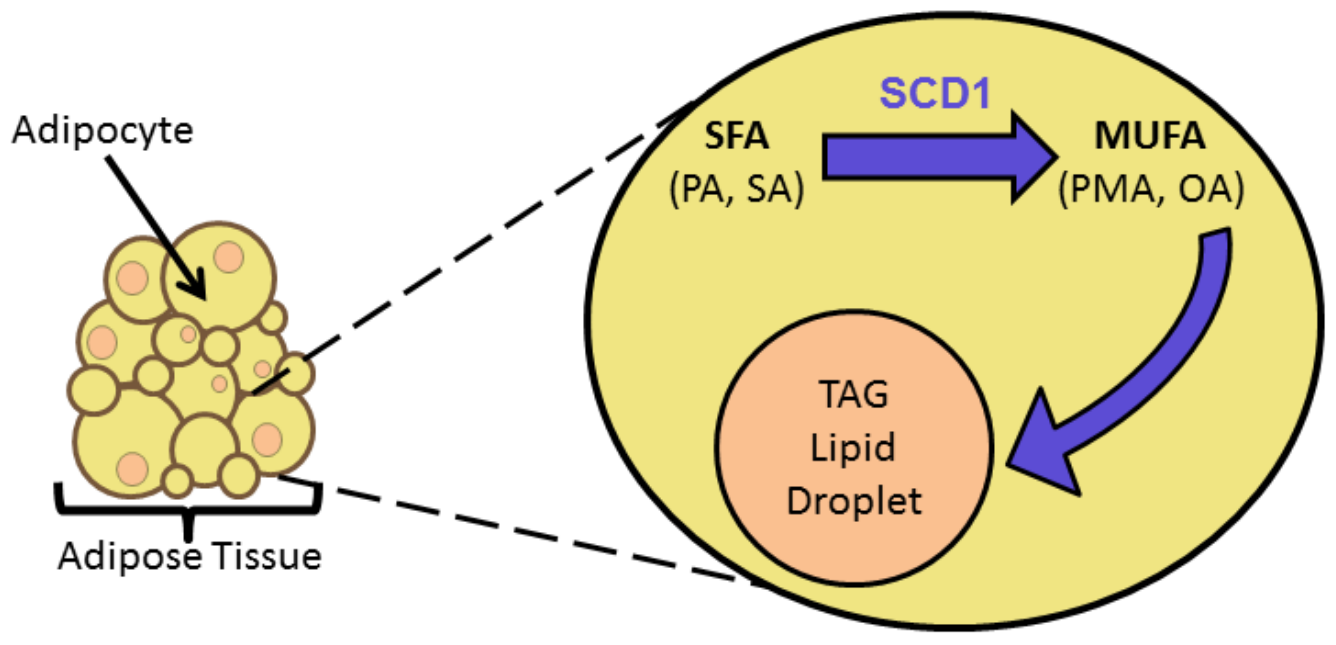

Figure 1.2: The MUFAs produced by SCD1 are the predominant substrates used for TAG production in lipogenic tissues including the liver and AT. MUFAs, monounsaturated fatty acids; SCD1, stearoyl-CoA desaturase 1; TAG, triacylglycerol; PA, palmitate; SA, stearate; PMA, palmitoleate; OA, oleate. 
The second link between SCD1 and lipid biosynthesis comes from work done by Man et al. [101]. These authors performed co-localization experiments in HeLa cells and demonstrated that SCD1 and diacylglycerol acyltransferase 2 (DGAT2) co-localize on the endoplasmic reticulum [101]. Two DGAT isoforms in the liver and AT (DGAT1 and DGAT2) produce the majority of endogenously synthesized TAGs [103, 104]. DGAT2 seems to be more involved in the esterification of de novo synthesized FAs to diacylglycerol (DAG) [103, 105], whereas DGAT1 appears to act downstream of DGAT2 and uses exogenous FAs [104]. Therefore, Man et al. suggested that dietary or endogenously-derived PA and SA are desaturated by SCD1 and then preferentially channelled over to DGAT2 for TAG production [101]. Reduced SCD1 activity would thus limit the efficiency of SCD1/DGAT2 FA-channelling for TAG synthesis. In addition, previous work has noted that SCD1 may regulate TAG synthesis simply by influencing levels of SFAs (which can inhibit FA synthesis) [106], and MUFAs (again, the main substrates for TAG production) $[9,77]$. However, the ability of SCD1 and DGAT2 to co-localize and act synergistically must be confirmed in other metabolic tissues such as AT.

Interestingly, hepatic SCD1 and DGAT2 are both present in a subcellular compartment known as the mitochondria-associated membrane, which is an ER domain enriched with enzymes involved in TAG and PL biosynthesis [101]. Based on these findings, it is plausible that in addition to DGAT2, SCD1 could function alongside other enzymes required for TAG and PL biosynthesis [101]. Supporting evidence for this notion includes work examining hepatic acylCoA cholesterol acyltransferase (ACAT) and glycerol-3-phosphate acyltransferase (GPAT), which are ER membrane enzymes that catalyze the synthesis of CEs and TAGs, respectively [11]. Miyazaki et al. have suggested that SCD1-derived MUFAs in the vicinity of ACAT and GPAT, but not exogenous MUFAs, could serve as preferential substrates for CE and TAG 
synthesis in the liver [11]. Additional experiments will determine if SCD1 in adipocytes also cofunctions with ACAT, DGAT2 and GPAT using a combination of inhibitors and activators for each enzyme, and measuring enzyme activities as well as the production of TAGs and CEs.

\subsubsection{The Influence of SCD1 on Adipocyte Differentiation and Cell Proliferation}

Adipocyte differentiation (i.e., adipogenesis) is a coordinated and complex process that involves the up-regulation of genes involved in lipid biosynthesis [7]. In order to study adipogenesis, researchers frequently use the established 3T3-L1 preadipocyte cell line [7, 107, 108]. The differentiation of 3T3-L1 preadipocytes involves the activation of C/EBP- $\alpha$ and PPAR $\gamma$, which assist in the induction of genes involved in adipocyte growth and lipid accumulation (e.g., Scd1, Fas, Srebp-1, glucose transporter 4 (Glut4), leptin, and fatty acid binding protein $4(F a b p-4))$ [7, 108, 109]. From this point onwards, $S c d 1$ expression and activity continue to rise substantially throughout terminal differentiation $[53,110]$. Tabor et al. have documented 20-fold and 1.8-fold increases in the expression of $S c d 1$ and $S c d 2$, respectively, throughout 3T3-L1 differentiation [81]. Kim et al. have also noted that Scd1 mRNA as well as SCD1 activity and protein content can increase as high as 100-fold during 3T3-L1 differentiation [53]. The substantial up-regulation of $S c d 1$ enables large increases in the endogenous production of MUFAs that are subsequently incorporated into TAG molecules [66, 101, 111]. These MUFA-enriched TAGs contribute to the production of large lipid droplets within growing adipocytes. Taken together, significant increases in $S c d l$ expression and activity, as well as the cellular accumulation of MUFA, are important for lipid handling in growing adipocytes; however, work by Christianson et al. partially challenges this dogma [109]. 
Christianson et al. examined the reliance of 3T3-L1 preadipocyte differentiation on Scd1 and $S c d 2$ expression and activity [109]. They used small inhibitory RNA (siRNA) oligonucleotides targeted towards 24 different genes involved in FA metabolism, including Scd1 and $S c d 2$. Of all the candidates examined, they reported that only $S c d 2$ depletion inhibited adipogenic gene expression (e.g. Ppary and Fas). Interestingly, $S c d l$ depletion did not reduce the expression of Ppary or Glut4, which are both critical for 3T3-L1 adipogenesis [109]. Therefore, $S c d 2$ may be more critical than $S c d 1$ for the induction of adipogenic genes and subsequent lipid accumulation [109]. Nonetheless, observations by Yee et al. suggest that SCD1 activity promotes the use of glucose for FA biosynthesis as well as cell survival during 3T3-L1 proliferation and differentiation [112]. Future work examining the reliance of 3T3-L1 differentiation on SCD1 or SCD2 activity is still needed, and could consider using enzymatic inhibitors or small inhibitor RNA (siRNA) to specifically reduce enzyme activity, and could also consider assessing if the importance of SCD1 or SCD2 changes at various time points of differentiation.

\subsubsection{The Influence of SCD1 on Fatty Acid Oxidation and Energy Expenditure}

While the protection of $S c d 1$-/- mice from diet-induced obesity could partially stem from reductions in FA biosynthesis and lipogenesis, another factor that potentially contributes to the prevention of obesity in these mice is an increase in FA oxidation [101]. The livers of Scd1-/mice compared to wild type mice have elevated expression of genes involves in FA oxidation, including acyl-CoA oxidase (Acox), very long chain acyl-CoA dehydrogenase (Acadvl), carnitine

palmitoyltransferase 1 (Cpt1), and fasting induced adipocyte factor (Fiaf/Angptl4) [15]. BAT from $S c d 1$-/- mice also displayed higher rates of FA oxidation compared to BAT from wild type 
mice [113]. Furthermore, AMP-activated protein kinase (AMPK) activity was increased in Scd1/- mice compared to wild type controls [100]. The phosphorylation of AMPK subsequently deactivates ACC by phosphorylation and contributes to reduced levels of malonyl-CoA [100]. A reduction in malonyl-CoA lessens the inhibition of CPT1, and therefore increases FA transport into the mitochondria for FA oxidation [100]. Indeed, Dobryzn et al. demonstrated that the livers of Scd1-/- mice have increased AMPK activity and ACC phosphorylation, reduced malonyl-CoA content, and increased CPT1 activation and PA oxidation in mitochondria [100]. In addition, Scd1-/- mice have higher Cptl expression and activity in BAT [113]. Moreover, AMPK activation reduced hepatic Srebp-1c expression [114], which could further contribute to the reduction in lipogenesis seen in Scdl-/- mice. Hulver et al. have also shown that high SCD1 activity (measured using a radioactivity assay) in obese human skeletal muscle can partition FAs away from FA oxidation and towards esterification into TAGs [41]. Along these lines, additional work has suggested that SFAs can reduce malonyl-CoA levels by inhibiting ACC [10]. In this way, SCD1-deficiency could lead to the accumulation of SFAs, which could reduce malonylCoA content and subsequently promote FA oxidation.

Along with increases in FA oxidation, SCD1-deficiency may cause an increase in energy expenditure and oxygen consumption. For example, $S c d 1 / /$ mice had higher rates of oxygen consumption compared to wild type mice when fed either a standard chow or high fat diet [15]. The authors attributed these higher rates of oxygen consumption to increased fat oxidation [15]. While the exact mechanisms that caused the increases in AMPK, energy expenditure and FA oxidation in Scd1-/- mice remain unknown [28], potential contributing factors include defects in the skin lipid barrier that allowed excess heat dissipation [115], as well as increased basal thermogenesis and expression of uncoupling proteins (Ucp) in BAT [10, 113]. BAT from Scd1-/- 
mice has increased Ucpl-3 gene expression, which suggests that $S c d 1$ in AT has a role in regulating metabolic rate and adiposity [113]. Taken together, the up-regulation of FA oxidation in $S c d 1$-/- mice adds uncertainty to whether a reduction in lipogenesis is the driving factor that protects these mice from diet-induced obesity, and highlights that the protection against adiposity may stem from increased energy expenditure.

\subsubsection{The Influence of SCD1 on Inflammation}

The existing evidence regarding the influence of SCD1 in inflammation has been somewhat inconsistent [18]. Some results suggest that there is a pro-inflammatory effect associated with SCD1-deficiency, while other evidence supports an anti-inflammatory association. Studies supporting a pro-inflammatory effect of SCD1-deficiency include a demonstration in dyslipidemic mice lacking the low-density lipoprotein receptor (Ldlr-/-) which were crossed with $S c d 1$-/- mice (i.e., Scd1-/-Ldlr-/- mice) [17]. These mice had elevated systemic inflammation (e.g., increased plasma IL-6, IL-1 $\beta$ and IL-12p70) compared to $S c d 1+/+L d l r-/-$ mice [17]. SCD1 inhibition in mice using antisense oligonucleotide injections has also been shown to elicit a pro-inflammatory response in isolated macrophages, which can promote atherosclerosis [19]. AKO mice also have elevated tumor necrosis factor $\alpha(\operatorname{Tn} f \alpha)$ expression in AT and reduced plasma adiponectin levels [27], while skin-specific Scdl knockout (SKO) mice exhibit increased cutaneous inflammation [116]. Furthermore, the development of inflammatory conditions such as ulcerative colitis was shown to involve the down-regulation of Scdl expression in mice [117]. Collectively these studies demonstrate that reduced SCD1 activity has pro-inflammatory effects, which is likely due to the accumulation of SFAs. For instance, Brown et al. proposed that a reduction in SCD1 activity contributes to elevated levels of SFAs in plasma 
low-density lipoprotein (LDL) particles as well as increased SFAs in macrophages, which ultimately enhanced secretion of pro-inflammatory cytokines from macrophages [19].

Additional evidence that SCD1 desaturation of SFAs has anti-inflammatory effects include a report that $S C D 1$ over-expression in primary human myotubes prevented PA-induced increases in the expression of inflammatory cytokines and markers of ER stress (i.e., $I L-8$, chemokine (C-X-C motif) ligand 3, activating transcription factor 3, and X-box binding protein 1) [69]. Other work has shown that $S C D 1$ up-regulation protected human arterial endothelial cells from PA-induced lipotoxicity and apoptosis, as well as prevented the induction of inflammatory cytokine expression (i.e., $I L-6$ and $I L-8$ ) [119]. Together these studies provide evidence of an anti-inflammatory role for SCD1 (or, conversely, a pro-inflammatory effect of SCD1-deficiency), which is somewhat intuitive since SCD1 converts SFAs to MUFAs and thereby prevents SFA accumulation and elevations in inflammation. However, findings from other investigations conflicts with these results.

Evidence supporting an anti-inflammatory effect of SCD1-deficiency includes work by Liu et al. showing that Scdl-/- mice fed a high-fat diet had reduced inflammation and inflammatory gene expression (i.e., $M c p-1, \operatorname{Tnf}-\alpha$ and CC-chemokine receptor $2(\mathrm{Ccr} 2)$ ) in AT compared to wild type mice fed the same diet [120]. This same investigation also demonstrated that the lipopolysaccharide (LPS) induced up-regulation of inflammatory cytokine gene expression (i.e., $M c p-1$ and $I l-6$ ) was reduced in adipocytes isolated from epididymal AT of Scd1-/- mice compared to wild type mice [120]. Furthermore, Hyun et al. showed that Scd1-/mice had higher adiponectin expression as well as lower Tnfa expression in AT compared to wild type mice when fed a high fat diet [27]. In human plasma, the PMA/PA desaturation ratio (a common estimate of SCD1 activity calculated using the enzyme product-to-precursor ratio) in 
the $\mathrm{CE}$ fraction was positively associated with circulating $\mathrm{C}$-reactive protein (CRP; a marker of systemic inflammation) levels $[121,122]$. Evidently, the impact of SCD1 activity or expression on inflammation varies by tissue and cell type, as well as the model in which SCD1 activity is altered. This likely contributes to the uncertainty regarding the relationship between SCD1 and inflammation.

\subsubsection{Other Physiological and Metabolic Effects of SCD1 Activity}

In addition to its roles in de novo lipogenesis, adipogenesis, FA oxidation and inflammation, SCD1 activity has the potential to alter cell membrane fluidity [66]. SCD1-derived MUFAs are the predominant constituents of membrane PLs [66]. Therefore, if a change in SCD1 activity alters the membrane SFA:MUFA ratio, this has implications for membrane fluidity and cell signalling cascades which can influence parameters such as insulin sensitivity $[11,123,124]$. Collins et al. used human adipocytes and demonstrated that SCD1 knockdown increased the SFA:MUFA ratio in PL, which reduced cell membrane fluidity [125]. Moreover, the reduction in membrane fluidity contributed to impaired insulin signalling [125].

SCD1 activity is also able to influence skin homeostasis and eye lubrication [30, 102]. SCD1-derived MUFAs are common substrates for the wax esters, TAGs and CEs present in the lipid lubricants secreted from sebaceous and meibomian glands (i.e., sebum and mebum) of the skin and eyelids $[10,32,126,127]$. Therefore, as expected, the eyelids and skin of Scdl-/- mice have lower levels of wax esters, TAGs and CEs when compared to wild type mice [29]. Consequently, Scdl-/- mice display atrophic sebaceous and meibomian glands, narrow eye fissure and cutaneous abnormalities [29], Furthermore, the inability of Scd1-/- mice to endogenously synthesize OA (i.e., the primary FA substrate for ACAT-derived CEs) can result 
in elevated free cholesterol levels that can cause cell death and atrophy of the sebaceous and meibomian glands [29, 32]. The eyes are also lubricated by alkyl-2,3-diacylglycerol (ADG) that is secreted from the Harderian gland, which is primarily composed of eicosenoate (20:1n-9; an elongation product of SCD1-derived OA) [30, 32]. ADG content in the Harderian gland is reduced in Scdl-/- mice [30], which likely also contributes to the eye abnormalities in these mice and thus highlights the importance of SCD1 activity for skin and eye health.

Finally, SCD1 also has a role in the formation of very-low density lipoproteins (VLDLs) in the liver. $S c d 1$-/- mice display a reduction in hepatic VLDL assembly and VLDL-TAG content [11], possibly due to their inability to produce MUFA-enriched TAGs that are the primary component of VLDLs [128, 129]. This could potentially impede the ability of the liver to secrete TAG; however, Scd1-/- mice appear to be more resistant to hepatic steatosis than wild type mice when fed a high-fat diet [15]. Elevated plasma VLDL-TAGs are associated with cardiovascular disease (CVD), and therefore a reduction in SCD1 activity could be beneficial for controlling VLDL-TAG production and secretion, as well as the development of CVD [16].

\subsection{SCD1 Activity at the Whole-Body, Adipose Tissue and Adipocyte Levels}

\subsubsection{Estimated Whole-Body SCD1 Activity using Blood Fatty Acid Ratios}

SCD1 activity is often estimated in human plasma or serum using FA product-toprecursor ratios, i.e., OA/SA (SCD-18) and PMA/PA (SCD-16) [10, 130-132]. These ratios are estimates of the combined activity of SCD1 and SCD5 in humans; however, the low levels of SCD5 in most tissues cause the ratios to predominantly estimate SCD1 activity [37, 42]. Using these estimates of enzymatic activity, studies in humans have investigated associations between 
SCD1 and markers of health and disease. Generally, the SCD desaturase ratios in plasma are inversely associated with insulin sensitivity and positively associated with the metabolic syndrome [131, 133]. Other studies have observed that higher plasma SCD-18 and SCD-16 ratios were associated with elevated plasma TAG levels in humans and mice [130, 134]. For example, Attie et al. placed human participants on a high carbohydrate diet (61-65\% of total energy intake) for 4-6 weeks and found that those who had elevated serum TAG levels also demonstrated elevated SCD-18 ratios in plasma [130]. In addition, the plasma SCD-16 ratio has been positively associated with body mass index (BMI), sagittal abdominal diameter and waist circumference in middle-aged adults [135].

Another parameter that has been associated with estimated SCD1 activity in plasma is inflammation. Petersson et al. demonstrated that higher SCD-16 ratios in serum CEs were positively associated with plasma CRP (a marker of systemic inflammation) in a population of older men [121]. Additionally, Stryjecki et al. observed a positive correlation between plasma CRP and plasma SCD-18 ratios in European and Asian female adults [136]. Furthermore, they found that a single nucleotide polymorphism (SNP) in the $S C D 1$ gene was inversely associated with plasma CRP [136].

Indeed, several studies have shown that plasma SCD-16 and SCD-18 ratios are associated with disease risk factors including inflammation, obesity, metabolic syndrome, hypertriglyceridemia, and insulin resistance; however, it is important to note the limitations involved with estimating SCD1 activity using FA ratios in plasma [132]. Plasma FA desaturation ratios reflect both endogenous FA metabolism as well as dietary fat intake [131]. SCD1 is also highly expressed in both the liver and AT, and therefore it is particularly challenging to delineate which tissue may be driving the plasma desaturation ratios and the associated correlations with 
disease parameters. Other factors that may confound the estimation of SCD1 activity using human plasma include genetic diversity (e.g., SNPs) and sex. For example, an examination of Swedish adults demonstrated that various SNPs in the SCDI gene were associated with improved insulin sensitivity as well as reduced waist circumference and BMI [137]. Regarding sex-differences, women may have higher plasma SCD-16 and SCD-18 ratios when compared to men [135]. Studies examining SCD-16 and SCD-18 ratios in human plasma or serum should therefore strive to account for genetic variation, physical activity and sex. While considerably more invasive, better estimations of SCD1 activity could be made using labelled FA substrates (e.g., U- ${ }^{13} \mathrm{C}-\mathrm{PA}$ and $\mathrm{U}_{-}{ }^{13} \mathrm{C}-\mathrm{SA}$ ) and measuring enzymatic activity using tissue biopsies.

\subsubsection{General Overview of SCD1 in Adipose Tissue Models}

In humans, de novo lipogenesis occurs predominantly in the liver; however, both AT and the liver are significantly involved in murine de novo lipogenesis [10, 23, 138]. Furthermore, Scdl is highly expressed in murine AT [10, 23, 138], and SCD1 expression in human AT is considerably high compared to any other tissue [139]. Taken together, this highlights the relevance of examining $S C D 1$ in both human and rodent AT. AT is a heterogeneous connective tissue composed of an adipocyte fraction and a stroma vascular fraction [140]. While the adipocyte fraction consists solely of mature adipocytes, the stroma vascular fraction contains numerous cell types including preadipocytes, macrophages, endothelial cells, fibroblasts, T-cells, and mesenchymal stem cells [141, 142]. Importantly, these cell types, as well as adipocytes, all influence AT metabolism and inflammation by secreting proteins referred to as adipokines [140$143]$. 
The structured vasculature of AT enables adipokines (e.g., cytokines) as well as nonesterified FAs liberated from AT, to influence biological processes throughout the body (e.g., inflammation, insulin sensitivity, lipid metabolism, energy balance, angiogenesis, appetite and immunity); these attributes contribute to the endocrine capabilities of AT [142-144]. In addition to its endocrine functions, the primary purpose of AT is to store excess calories in the form of TAG within the lipid droplets of adipocytes [142, 143]. This implicates adipocytes in the development of obesity, and highlights the importance of studying lipid metabolism within this cell type. Given the links between SCD1 and TAG biosynthesis, understanding the influences of SCD1 activity in AT metabolism may also be important in the study of obesity.

Prior to discussing the relationships between AT and SCD1, it is important to first acknowledge the ability for exogenous factors (e.g. dietary factors) to alter $S c d 1$ expression and activity in murine and human AT. For instance, dietary PUFAs have been shown to reduce $S c d 1$ expression in abdominal AT of lean and obese Zucker rats [145]. Additionally, the SCD-16 ratio in adipose venous non-esterified FAs (which represent subcutaneous AT) was elevated by a high carbohydrate diet compared to a high fat diet in healthy adults [60]. Conversely, rosiglitazone has been demonstrated to increase the plasma SCD-16 ratio as well as SCD1 expression in subcutaneous AT from type 2 diabetic adults [65]. Caloric or food restriction also appears to influence AT Scdl expression. For example, prolonged food restriction (1 month) in rats has been shown to increase $S c d l$ expression in subcutaneous, perirenal and epididymal AT compared to control animals [146]. In this same study, Scdl expression in the AT of the chronic food restricted rats was even further increased by re-feeding for 48 hours (to levels above those seen with chronic food restriction) [146]. Mainieri et al. additionally found that Scdl expression in retroperitoneal AT was reduced by semi-starvation in rats, but $S c d l$ expression in epididymal AT 
was unaltered when compared to control-fed rats [147]. In humans, Mangravite et al. saw that acute weight loss reduced SCDI gene expression in subcutaneous AT from overweight men [148]. Collectively, it appears as though a broad list of exogenous factors are able to influence Scdl expression and activity in AT.

The existing evidence regarding how Scdl expression and SCD1 activity can influence obesity-related complications (e.g., insulin resistance) in AT is limited [132]. However, significant contributions to our understanding of SCD1 in AT have been made using human, rodent and cell models. The following sub-sections will highlight the key research that has been conducted in these models.

\section{i) SCD1 in Human Adipose Tissue}

SCD1 expression and activity in human AT has been correlated with clinical parameters including insulin resistance and obesity $[149,150]$. Sjögren et al. examined subcutaneous AT in men and showed that a high SCD-18 ratio was associated with insulin resistance [149]. Subcutaneous and visceral AT from morbidly obese (BMI > $50 \mathrm{~kg} / \mathrm{m}^{2}$ ) individuals had a much higher SCD-18 ratio (but not SCD-16 ratio) when compared to AT from participants classified as overweight $\left(\mathrm{BMI}>27.5 \mathrm{~kg} / \mathrm{m}^{2}\right)$ [99]. Interestingly, while SCD1 protein content was also higher in AT from morbidly obese participants, $S C D 1$ gene expression was lower [99]. Caron-Jobin et al. have previously noted that the SCD-18 ratio in subcutaneous AT was positively correlated with markers of obesity (i.e., BMI and subcutaneous AT area) in women [118]. Additional work supporting a link between SCD1 activity and obesity was demonstrated by Gong et al, where SCD-16 and SCD-18 ratios in subcutaneous AT were positively associated with obesity [151]. In regards to the relationship between insulin resistance and SCD1 activity, studies have observed 
that the SCD-18 ratio in subcutaneous AT (but not the SCD-16 ratio) was positively correlated with insulin resistance $[149,150]$.

Investigations of SCD1 in human AT have mainly been done using subcutaneous AT $[132,149,150]$, primarily because this is an accessible and less invasive AT depot to collect compared to depots surrounding internal organs (e.g., visceral AT). One study that has examined visceral omental AT of female participants found a positive correlation between the SCD-18 ratio and plasma TAG levels [118]. Furthermore, visceral AT may be more metabolically and lipolytically active due to higher content of inflammatory cells (e.g., macrophages) when compared to subcutaneous AT [152-154]. Therefore the assessment of subcutaneous AT compared to visceral AT may be limited in the ability to investigate metabolic parameters. Another limitation to examining subcutaneous AT includes the variability of SCD1 expression and activity in different subcutaneous AT depots throughout the body. Pinnick et al. observed that subcutaneous gluteal AT from human participants had higher SCD-16 and SCD-18 ratios as well as higher SCD1 gene expression, when compared to subcutaneous abdominal AT [155]. Other factors that can potentially confound the measurement of SCDI expression in subcutaneous AT are sex-specific differences. For instance, females may have higher SCD1 expression in subcutaneous abdominal and gluteofemoral depots compared to men [155]. Finally, ATPase p97 (which is implicated in the regulation of SCD1 via proteasome-dependent protein degradation) is more highly expressed in visceral AT compared to subcutaneous AT [99], which suggests that SCD1 turn-over may be differentially regulated in different AT depots. Therefore, future work investigating SCD1 expression or activity in human AT should consider examining various AT depots, and should account for confounding factors including sex. 


\section{ii) SCD1 in Rodent Adipose Tissue}

The use of rodent models has facilitated the examination of $S c d l$ expression in AT. While $S c d 1$ is generally constitutively expressed in rodent AT [45, 66], studies examining $S c d 1$ have often used genetic, dietary or chemical means to alter $S c d l$ expression and activity [145, $156,157]$. Genetic models have been used to confirm that the positive association between Scd1 and obesity holds true in rodent AT, which is important for the ability to translate results from rodent studies to humans. For instance, $S c d l$ expression in abdominal AT from obese Zucker rats was substantially elevated compared to AT from lean rats [145].

Studies investigating $S c d 1$ in rodent AT include the use of antisense oligonucleotide injections targeted towards $S c d l[19,156,158]$. This work has demonstrated that reductions in Scdl expression in both the AT and the liver could improve insulin sensitivity [19, 156, 158]. In a separate study, Scd1-/- mice fed a high-fat diet had improved basal insulin signalling in epididymal AT compared to wild type mice $[120,159]$. This same study also showed that epididymal AT from $S c d 1$-/- mice fed the same high-fat diet had reduced inflammation when compared to wild type AT. Specifically, they saw a reduction in AT cell death, as well as decreased expression of Mcp-1, Tnfa and vascular cell adhesion molecule 1 (Vcam-1) [120]. Together, these investigations suggest that reduced $S c d 1$ expression at the whole-body level may improve insulin sensitivity and the inflammatory response of AT. However, models entailing whole-body SCD1 deficiency (i.e., Scd1-/- mice or antisense oligonucleotide injections) are limited in their ability to deduce if the observed metabolic changes are AT-specific, or due to other underlying mechanisms in another tissue. For instance, when whole-body $S c d l$ is reduced, any changes occurring in the liver and skeletal muscle could influence adipocyte metabolism 
through the secretion of signalling molecules (e.g., cytokines or lipids) [160] which in turn could confound conclusions made about AT.

Significant contributions to our understanding of $S c d 1$ in AT have been made using AKO mice. These mice display similar $S c d l$ expression in the liver and skeletal muscle as wild type animals; however, $S c d 1$ expression and protein content are substantially reduced (by 90-95\%) in subcutaneous and epididymal AT [27]. AKO mice exhibit reduced adiponectin expression and secretion, but elevated Tnf $\alpha$ expression compared to wild type mice. Additionally, the expression of Glut1 was up-regulated in AT of AKO mice [27], suggesting enhancements in basal insulin signaling and glucose transport. Feeding AKO mice a chow diet reduced Glut4 expression compared to control mice, yet feeding a high-fat diet did not differentially alter Glut4 [27]. This differed from observations in $S c d 1$-/- mice (i.e., mice with whole-body $S c d 1$ deficiency), where Scd1-/- mice had higher Glut4 expression in AT compared to wild type mice on either a chow or high-fat diet; however, their Glut1 expression was unaltered [27]. This work comparing the AT of AKO and Scd1-/- mice demonstrates that adipose-specific vs. whole-body Scdl deficiency differentially influences glucose transporters in AT. Furthermore, this likely indicates that compensatory effects in other tissues are contributing to the variability seen in the AT of Scdl-/mice compared to AKO mice, which again highlights a limitation of using whole-body rodent models to examine $S c d 1$ in AT.

In addition to differences in glucose transporters, the phenotype of AKO mice also diverts significantly from $S c d 1$-/- mice and from liver-specific $S c d 1$-/- (LKO) mice, which are also protected from diet-induced adiposity. Specifically, AKO mice were not protected from adiposity when fed a high carbohydrate diet, unlike $S c d 1-/-$ and LKO mice [27]. Additionally, whole-body glucose tolerance was unaltered in AKO mice compared to control mice [27], 
suggesting that $S c d l$ deficiency in AT did not provide the same insulin sensitizing effects as whole-body Scdl deficiency. Finally, the cytokine expression profile in the AT of AKO mice was significantly different from Scdl-/- mice. When fed a chow diet AKO mice had reduced adiponectin expression and elevated Tnfa expression compared to control mice [27]. Conversely, Scd1-/- mice had higher adiponectin expression in AT as well as plasma adiponectin levels compared to wild type mice when fed the same chow diet [27]. Interestingly, although a high-fat diet increased Tnfa expression in wild type mice, this increase was significantly blunted in Scd1/- mice, but not in AKO mice. Discrepancies between AKO and Scd1-/- mice have therefore created some uncertainty regarding the influence of SCD1 activity in AT. Adding further to this ambiguity, mice with a combined knockout of $S c d l$ in the liver and AT (i.e., LAKO mice) were not protected from diet-induced adiposity, unlike $S c d 1$ /- and LKO mice, but similar to AKO mice [160]. The high expression of $S c d l$ in AT and the significant changes in AT metabolism that occur in models of $S c d 1$ deficiency (e.g., Scdl-/-, LKO, AKO, LAKO, and mice treated with antisense oligonucleotides), emphasize the importance of understanding exactly how $S c d l$ evokes changes in AT metabolism. The molecular mechanisms contributing to the ability of SCD1 to impact AT metabolism can be investigated using adipocyte cell culture models.

iii) SCD1 in Adipocyte Models

The use of primary adipocytes and adipocyte cell lines enables the investigation of the biological processes occurring specifically within adipocytes, without the exogenous influences of other cell types within AT. This is important, as it can advance our understanding of what drives the effects seen at the whole-body level (e.g., Scd1-/- mice). Studies focused on primary adipocytes isolated from omental and subcutaneous AT of human adults have shown that SCD1 
expression was conserved in obese adipocytes, but reduced in morbidly obese adipocytes compared to lean adipocytes [161]. In a separate study, Liu et al. isolated primary adipocytes from $S c d 1$-/- mice and wild type mice. They found that adipocytes from $S c d 1$-/- mice, compared to wild type mice, had reduced expression of $M c p-1$ and $I l-6$, but unaltered expression of other genes including Ppary and Ppary target genes (e.g., Pepck, Glut4, Fabp4), Cpt1, and genes involved in the regulation of FA oxidation, oxidative stress, and mitochondrial metabolism [120]. Furthermore, Liu et al. challenged adipocytes isolated from Scd1-/- mice and control mice with lipopolysaccharide (a TLR-4 ligand known to induce inflammation), and found that the induction of $M c p-1$ and $I l-6$ was reduced in adipocytes from $S c d 1-/-$ mice compared to wild type mice [120]. They also collected adipocyte-conditioned media (i.e., media containing adipocytesecreted factors including lipids and cytokines). This media was applied to RAW264.7 macrophages, and they observed that the induction of Tnf $\alpha$ and $I l-1 \beta$ expression was lower in macrophages treated with media from adipocytes of $S c d 1$-/- mice compared to wild type mice [120]. Liu et al. concluded from these findings that a reduction in whole-body Scdl expression can reduce inflammation in adipocytes as well as the regulation of macrophage inflammation by adipocytes [120]. However, because the adipocytes examined by Liu et al. were from Scd1-/mice, the results could be confounded by compensatory effects in other tissues (or other cell types within AT) that occurred throughout the lifespan of the mice, i.e., prior to the isolation of adipocytes.

Adipocyte cell culture models can be a suitable method for examining Scdl expression or activity in adipocytes without the influence of exogenous factors from other tissues or cell types. Jones et al. used the 3T3-L1 adipocyte model to demonstrate that PUFAs (i.e., linoleate) significantly down-regulated Scdl expression [145]. Sessler et al. also observed that FAs 
including arachidonate, linoleate, $\alpha$-linolenate and eicosapentaenoate reduced $S c d 1$ expression in 3T3-L1 adipocytes [71]. Adipocyte cell culture models have also been used to manipulate SCD1 activity using chemical inhibitors, which enables the direct assessment of how reduced SCD1 activity influences adipocyte metabolism. For instance, Hyun et al. examined glucose uptake in 3T3-L1 adipocytes treated with an SCD1 inhibitor (A939572; Biofine International), and found (as expected) that SCD1 inhibition reduced PMA and OA content relative to control adipocytes [27]. In addition, although basal glucose uptake was enhanced in SCD1-inhibited cells compared to control cells, insulin-stimulated glucose uptake was not affected. They also noted that the elevation in basal glucose uptake in SCD1-inhibited cells occurred concomitantly with an increase in Glut1 expression and protein content, but Glut4 expression was unaltered [27]. In this same study, Hyun et al. also observed that adiponectin expression was reduced in SCD1inhibited 3T3-L1 adipocytes compared to control cells [27], which may suggest that inhibiting SCD1 activity in adipocytes has pro-inflammatory effects.

Malodobra-Mazur et al. used the same SCD1 inhibitor as Hyun et al. to demonstrate that SCD1 influences inflammation through altered DNA methylation [162]. Moreover, they also observed a reduction in total TAG and the SCD-18 desaturation ratio in SCD1-inhibited adipocytes [162]. Additional studies that have used inhibitors or other techniques to reduce SCD1 activity in adipocyte models include work by Yee et al [112]. These authors observed that the SCD1 inhibitor CGX0290 (CompleGen Inc.) in 3T3-L1 adipocytes caused a reduction in the use of glucose for FA synthesis and increased cell death [112]. Kim et al. also examined the impact of SCD1 inhibition in 3T3-L1 adipocytes [163]. They noted that SCD1 inhibition (using A939572) decreased the expression of lipogenic genes (e.g., Fas, Acc, Srebplc, Ppary) and lipid accumulation, increased AMPK activation, but did not alter the expression of genes for FA 
oxidation (Cpt1, Ucp2, Ppar $)$ [163]. Other work has made use of another inhibitor (CAY10566; Cayman Chemical) to show that SCD1 inhibition impaired cell proliferation of NIH-3T3 fibroblasts [164], and induced the activation of p38 mitogen-activated protein kinase (MAPK) and ER stress [165]. Direct knockdown of SCD1 in human adipocytes using siRNA has also been performed by Collins et al., and they saw that this caused a decrease in cell membrane fluidity due to an increased SFA:MUFA ratio in PL [125]. They also observed that SCD1 knockdown caused a reduction in insulin-stimulated glucose uptake [125], suggesting that $S C D 1$ in human adipocytes may be necessary for the maintenance of insulin sensitivity in AT.

Other arguably less specific methods of altering SCD1 activity in adipocytes include the use of thiazolidinediones, sterculic acid, and trans-10,cis-12 conjugated linoleic acid (CLA) [53, 166-168]. For instance, troglitazone treatment in 3T3-L1 preadipocytes dramatically reduced Scd1 mRNA levels without changing $S c d 2$ mRNA levels [53]. Sterculic acid (a plant lipid containing a propene ring) has also been documented to reduce SCD1 activity by $90 \%$ in 3T3-L1 adipocytes, which coincided with an increase in cell size but did not alter glucose uptake [167]. Despite larger cells, adipocytes treated with sterculic acid had fewer and smaller lipid droplets compared to control cells [167]. Similar results have also been seen in SCD1-inhibited 3T3-L1 cells (treated with CLA), where adipocytes had smaller lipid droplets compared to control cells [168]. The effects of sterculic acid- and CLA-induced SCD1 inhibition were also examined in primary bovine adipocytes, where both inhibitors where found to down-regulate genes involved in lipogenesis and up-regulate genes involved in lipolysis and FA oxidation [166]. Taken together, there are many means of inhibiting SCD1 activity in adipocyte models, and these inhibitors can be useful tools to assist with delineating the influences of SCD1 in adipocyte metabolism. 
It should be acknowledged that the use of isolated adipocytes or adipocyte cell culture models are somewhat limited in their ability to be extrapolated to whole body or human contexts, since cell culture is not accurately representative of whole body physiology. However, adipocyte cell culture models are still extremely useful for identifying potential mechanisms and pathways that may help guide future in vivo research. Furthermore, SCD1 inhibition in adipocytes can be a targeted approach for directly studying how reduced SCD1 activity influences adipocyte metabolism.

\subsection{Summary}

Through its role in converting SFAs to MUFAs, SCD1 is able to influence a myriad of metabolic processes including de novo lipogenesis, FA oxidation, inflammation, cell membrane fluidity and skin homeostasis. Over the past 25 years significant advancements in our understanding of hepatic $S c d l$ have been made, and much of this progress has stemmed from the examination of $S c d 1$-/- mice. Despite the ability of hepatic $S c d l$ expression and activity to influence de novo lipogenesis, which occurs predominantly in the liver, Scdl is also highly expressed in AT. The significant lipogenic capacity of AT combined with reports that human SCD1 expression is highest in AT, suggest that changes in SCD1 activity within AT could dramatically alter lipid biosynthesis and whole-body metabolism. While the evidence of how altered SCD1 activity in AT and adipocytes remains somewhat limited, significant developments have been made using AKO mice as well as adipocyte cell culture models. However, a thorough and complete understanding of how altered SCD1 activity influences AT and adipocyte biology 
remains necessary. For example, it is unclear to what extent an adipocyte-specific reduction in SCD1 activity can influence lipid metabolism, inflammation and insulin sensitivity.

Targeted Scdl knockdown and SCD1 inhibition techniques have been implemented in adipocyte cell models in an effort to investigate how SCD1 activity can influence global adipocyte biology. Previous reports have suggested that modifying SCD1 activity in adipocytes may influence insulin sensitivity, lipid metabolism, and inflammation. However, previous results have been somewhat inconsistent regarding the relationship between SCD1 activity and these aforementioned processes. For example, SCD1 activity has been observed to be both positively and negatively associated with adipocyte inflammation. This is significant, because SCD1 activity has been described as a potential therapeutic target for obesity interventions [169], primarily because $S c d 1$-/- mice are protected from diet induced adiposity. Accordingly, a more complete understanding of how altered SCD1 activity can influence adipocyte metabolism is required to advance our knowledge regarding this critically important enzyme. 


\section{CHAPTER 2:}

AIMS OF THESIS 


\section{$\underline{2.1 \text { Rationale and Overall Objectives }}$}

Over the past 20 years, significant advancements have been made in our knowledge of SCD1, with Scd1-/- mice in particular serving as a foundational model. These mice are protected against diet-induced obesity, and have reduced whole body adiposity and cellular lipid accumulation and insulin resistance $[11,15,16]$. However, the majority of SCD1 research has examined hepatic metabolism, primarily due to the high rates of de novo lipogenesis that occur in the liver, as well as the significant ability for dietary regulation of hepatic Scd1 expression [2326]. In contrast, our understanding of SCD1 in AT and adipocytes is incomplete despite the high expression of SCD1 in human and rodent AT and the purported links between SCD1 and obesity $[8,139]$. The few studies that have examined SCD1 in AT and adipocytes have provided important insights, but they have also, at times, generated inconsistent results. For instance, the AT of AKO mice had lower adiponectin expression and elevated expression of pro-inflammatory cytokines [27]. However, the AT of Scd1-/- mice had reduced markers of inflammation compared to wild type mice [27]. In order to improve our understanding of SCD1 in AT, the influences of SCD1 activity in adipocytes must be further delineated. Therefore, the overall objective of this thesis was to advance our knowledge of how altered SCD1 activity can influence adipocyte fatty acid metabolism and cellular function. The overall hypothesis of this thesis was that altered adipocyte SCD1 activity would modify cellular FA content, which would influence numerous aspects of adipocyte biology including lipid biosynthesis and metabolism, global gene expression, basal insulin signaling, inflammation and cellular stress. Three independent studies were conducted as a means of addressing this thesis objective and hypothesis. 


\subsection{Specific Study Objectives and Hypotheses}

$\underline{\text { Study } 1}$

The specific objectives of study 1 were to:

1. Examine how reduced SCD1 activity in differentiating adipocytes affects the profile of total lipids.

2. Examine how reduced SCD1 activity alters gene expression networks in adipocytes.

Given the objectives of study 1 , I hypothesized that:

1. Inhibiting SCD1 activity would reduce the endogenous production of MUFAs and cause an accumulation of SFAs in differentiated adipocytes.

2. SCD1 inhibition would cause a decrease in total TAG content, and that the examination of biological pathways and gene expression would shed light on the potential mechanism(s) contributing to lower TAG levels. This was based on previous reports showing that $S c d 1$-/- mice have lower TAG levels in the liver and AT.

$\underline{\text { Study } 2}$

The specific objectives of study 2 were to:

1. Determine the full extent to which SCD1 inhibition influences the content and FA composition of lipid fractions (i.e., PL, TAG, CE, FFA, DAG) of adipocytes throughout adipogenesis (i.e., adipocyte differentiation).

2. Determine how reduced SCD1 activity influences basal insulin signaling or cellular stress in adipocytes.

Given the objectives of study 2, I hypothesized that: 
1. SCD1 inhibition would induce changes in FA and lipid profiles early in the adipogenesis process. The rationale for this stemmed from the rapid and substantial increase in Scd1 gene expression that occurs during adipogenesis.

2. Total PL content would be reduced in SCD1-inhibited adipocytes. This was based on previous reports showing that SCD1 produces the MUFAs that are highly abundant in PLs.

3. SCD1 inhibition would improve basal insulin signaling. The reasoning for this was because $S c d 1$-/- mice have been documented to have improved whole-body glucose homeostasis.

\section{$\underline{\text { Study } 3}$}

The specific objectives of study 3 were to:

1. Identify how exogenous FAs related to SCD1 (i.e., PA, SA, PMA and OA) influence adipocyte lipid metabolism, cellular stress and inflammation.

2. Examine if SCD1 inhibition mediates the impact of exogenous FAs on the aforementioned processes.

Given the objectives of study 3, I hypothesized that:

1. SFA treatments would induce inflammatory markers and cellular stress in adipocytes, but MUFAs would not have these effects. This was based on the current dogma that SFAs are more lipotoxic and inflammatory than MUFAs.

2. Reduced SCD1 activity in adipocytes would cause a greater inflammatory and stress response to FAs in comparison to control adipocytes. This was based on the assumption 
that SCD1 inhibition causes SFA accumulation, which would exacerbate the effects of any exogenous FAs.

The methodologies and results associated with each of these studies are presented in the forthcoming chapters. 


\section{CHAPTER 3:}

\section{INHIBITION OF STEAROYL-COA DESATURASE 1 IN DIFFERENTIATING 3T3-L1 PREADIPOCYTES UPREGULATES ELONGASE 6 AND DOWNREGULATES GENES AFFECTING TRIACYLGLYCEROL SYNTHESIS}

As published in the International Journal of Obesity:

Jessica C. Ralston, Flavia Badoud, Bradley Cattrysse, Paul D. McNicholas, and David M. Mutch (2014). Inhibition of stearoyl-CoA desaturase-1 in differentiating 3T3-L1 preadipocytes upregulates elongase 6 and downregulates genes affecting triacylglycerol synthesis. Int $J$ Obesity. 38(11): 1449-1456. 


\subsection{Abstract}

Background: Stearoyl-CoA desaturase-1 (SCD1) is rate limiting for the conversion of saturated fatty acids palmitate (16:0) and stearate (18:0) to monounsaturated fatty acids palmitoleate $(16: 1 n-7)$ and oleate (18:1n-9), respectively. Given that reduced SCD1 activity is associated with improved insulin sensitivity and decreased body weight, there is considerable interest to elucidate the role of this enzyme in adipocytes. During adipogenesis, SCD1 levels increase concomitantly with the accumulation of triacylglycerol (TAG); however, the extent to which reduced SCD1 activity can influence TAG synthesis and metabolic pathways in differentiating adipocytes remains relatively unexplored.

Objective: The aim of this work was to delineate how reduced SCD1 activity affects gene expression, protein content and cellular fatty acids in differentiating murine preadipocytes.

Methods: 3T3-L1 preadipocytes were treated with an SCD1 inhibitor (10 $\mathrm{nM})$ throughout differentiation. After 7 days, global gene expression, protein content and fatty acid profiles were examined using microarrays, western blotting and gas chromatography, respectively.

Results: SCD1 inhibition increased the abundance of 16:0 and 18:0 (45\% and 194\%, respectively) and decreased 16:1n-7 and 18:1n-7 (61\% and 35\%, respectively) in differentiated preadipocytes. Interestingly, 18:1n-9 levels increased by $61 \%$. The augmented 18:0 suggested a possible increase in elongase activity. Elongase 6 (Elovl6) gene expression was increased 2.8fold $(\mathrm{P}=0.04)$; however, changes were not detected for ELOVL6 protein content. Microarray analysis revealed that genes affecting TAG synthesis were downregulated with SCD1 inhibition, which coincided with a 33\% decrease in cellular TAG content. 
Conclusion: These results provide new mechanistic insight into the role of SCD1 as a regulator of fatty acid profiles and TAG synthesis in adipocytes, and reinforce that modulating SCD1 activity may help reduce the risk of obesity-related complications.

\subsection{Introduction}

Obesity is a major worldwide health concern that is associated with complications such as type 2 diabetes, hypertension, cardiovascular disease, cancer, and metabolic syndrome [2]. Positive caloric balance is a major contributor to the development of obesity, where excess energy is stored as triacylglycerol (TAG) in the adipocytes of adipose tissue (AT) $[6,170]$. In an attempt to reduce the prevalence of obesity, it is of interest to delineate the complex molecular

events that regulate adipocyte function [7, 171]. Preadipocytes undergo coordinated and multifaceted changes in gene expression and protein content as they differentiate into mature adipocytes [7]. Many of the genes and proteins that are modified throughout differentiation are involved in the regulation of lipid metabolism [7]. Among the genes whose expression increases markedly throughout differentiation is stearoyl-CoA desaturase 1 (SCD1). SCD1 is the ratelimiting enzyme catalyzing the conversion of saturated fatty acids (SFA) palmitate (16:0) and stearate (18:0) to monounsaturated fatty acids (MUFA) palmitoleate (16:1n-7) and oleate (18:1n9), respectively [28]. Although several isoforms of SCD have been identified (SCD1 and SCD5 in humans, and SCD1-SCD4 in mice), SCD1 is the predominant isoform found in AT and has been shown to have a key role in lipogenic tissues [10, 28].

As adipocytes differentiate and mature, they develop large TAG-rich lipid droplets. Existing evidence indicates that the preferred fatty acid (FA) substrates for TAG synthesis are 
the MUFA produced endogenously by SCD1 [10, 101, 111]. Elongase 6 (ELOVL6), which converts $16: 0$ and $16: 1 \mathrm{n}-7$ to $18: 0$ and $18: 1 \mathrm{n}-7$, respectively, is also involved in FA biosynthesis [172]. As such, altering SCD1 or ELOVL6 activity during adipocyte differentiation has the potential to significantly modify the FA profile and, ultimately, TAG synthesis. Changes in FA profiles can affect many biological pathways in adipocytes through the regulation of gene expression [64]. As a result, varying FA abundance can affect processes such as inflammation, angiogenesis, and insulin sensitivity [64]. Taken together, altered SCD1 activity in adipocytes could have a profound effect on the development of obesity-related complications.

The purpose of the present study was to investigate the effects of SCD1 inhibition on FA profiles, as well as the underlying effects on gene and protein expression, in differentiating preadipocytes. We hypothesized that inhibiting SCD1 activity would not only reduce the endogenous production of MUFA and cause an accumulation of SFA precursors, but would also alter TAG synthesis pathways. Given the well-established relationship between SCD1 and obesity, this study provides new mechanistic insight that improves our understanding of how SCD1 activity influences lipid metabolism during adipogenesis. Ultimately, this research supports the notion that regulating SCD1 during adipogenesis could reduce the risk of obesityrelated complications.

\subsection{Methods}

\subsubsection{Chemicals and Cell Culture Reagents}

Cell culture reagents including Dulbecco's modified Eagle's medium (DMEM), 0.25\% trypsin-ethylenediamine tetraacetic acid solution, and penicillin-streptomycin (pen-strep) were 
purchased from Hyclone laboratories (Logan, UT, USA). Other cell culture reagents including 3isobutyl-1-mexthylxanthine (IMBX), dexamethasone (Dex), human insulin, fatty acid-free bovine serum albumin (BSA; $\geq 98 \%$ purity), fetal bovine serum, and dimethyl sulfoxide (DMSO; $\geq 99.9 \%$ purity) were purchased from Sigma Aldrich (St. Louis, MO, USA). Murine 3T3-L1 preadipocytes were obtained from ATCC (Rockville, MD, USA). A specific SCD1 inhibitor (CAY10566) was purchased from Cayman Chemical (Ann Arbor, MI, USA). SCD1 and peroxisome proliferator-activated receptor gamma (PPAR $\gamma)$ primary antibodies were purchased from Cell Signaling Technology (Danvers, MA, USA). ELOVL6 and $\alpha$-tubulin primary antibodies were purchased from Abcam (Toronto, ON, Canada).

\subsubsection{Cell Culture Experiments}

3T3-L1 adipocytes were cultured in $5 \% \mathrm{CO}_{2}$ and $100 \%$ humidity at $37^{\circ} \mathrm{C}$. Cells were maintained in basic media, which consisted of DMEM supplemented with 1\% pen-strep and 5\% heat-inactivated FBS. Cells were seeded at a density of $6.0 \times 10^{4}$ cells per well in six-well plates or at $1.3 \times 10^{5}$ cells per T25 flask. The SCD1 inhibitor ( $\mathrm{SCD} 1_{\text {inhib }}$ ) was diluted in DMSO to create a $10 \mu \mathrm{M}$ stock solution. A final working concentration of $10 \mathrm{nM} S C D 1_{\text {inhib }}$ was used in all adipocyte experiments. This concentration of $\mathrm{SCD} 1_{\text {inhib }}$ was selected based on dose-response experiments in order to identify the dose that maximized SCD1 inhibition while avoiding cell toxicity. Toxicity was assessed using the Promega Cytotoxicity Assay (Madison, WI, USA).

Differentiation was induced at day 0 (i.e., 2 days after confluence) with a standardized differentiation cocktail consisting of IBMX (0.5mM), Dex (1 $\mu \mathrm{M})$, and human insulin $(5 \mu \mathrm{g} / \mathrm{ml})$ in basic media, as described previously [64]. After 2 days (i.e., day 2) the media was changed to maintenance media, which consisted of basic media supplemented with human insulin $(5 \mu \mathrm{g} / \mathrm{ml})$. 
On day 4, FBS was removed from the media to achieve serum-free conditions for the remaining duration of the experiments. Throughout differentiation cells were treated with either $10 \mathrm{nM}$ SCD1 $1_{\text {inhib }}$ or an equivalent volume of DMSO (i.e., the control condition). All sample extractions were performed on day 7, with six technical replicates per treatment condition for all RNA and protein analyses, and three technical replicates for lipid analyses. In addition, all of the aforementioned experiments were replicated several times at different passage numbers to ensure that results were not due to passage number.

\subsubsection{Lipid Extraction and Quantification}

We used a modified Bligh and Dyer method for lipid extractions $[173,174]$. On day 7 of differentiation, media was removed, cells were washed with PBS 1X, and then cells were trypsinized for $5 \mathrm{~min}$. The solution was collected and spun at 1,200 rpm for 5 min to pellet adipocytes. The supernatant was discarded and the cell pellet washed in PBS 1X. A 2:1 methanol:chloroform solution (v/v) was added to cell pellets and samples were then vortexed for $5 \mathrm{~min}$. Next, a 1:1 chloroform:water solution (v/v) was added and samples were vortexed and centrifuged at 2,500 rpm for $20 \mathrm{~min}$. The organic phase was collected and dried under a gentle stream of nitrogen gas.

For total FA quantification a 1:1 methanol:chloroform solution containing $10 \%$ boron trifluoride-methanol $\left(\mathrm{BF}_{3}-\mathrm{MeOH}\right)$ was added to all samples before methylation for $1.5 \mathrm{hrs}$ at $100^{\circ} \mathrm{C}$. Samples were then cooled prior to the addition of double-distilled water and hexane.

For TAG quantification, $100 \mu \mathrm{L}$ of chloroform was added to the original dried organic extract. $100 \mu \mathrm{L}$ of each sample and $25 \mu \mathrm{L}$ of TAG standard were spotted on Silica-G thin-layer chromatography (TLC) plates set in a 20x5 cm TLC chamber containing $80 \mathrm{~mL}$ petroleum ether, 
$20 \mathrm{~mL}$ ethyl ether, and $1 \mathrm{~mL}$ acetic acid [175]. TLC plates were run for $45 \mathrm{~min}$ prior to TAG band visualization with $0.1 \%$ 8-anilino-1-naphthalene sulfonic acid [175]. TAG bands were collected before the addition of hexane and $14 \% \mathrm{BF}_{3}-\mathrm{MeOH}$, followed by $1.5 \mathrm{hrs}$ of methylation at $100^{\circ} \mathrm{C}$. Samples were then cooled and double-distilled water was added before centrifugation at $1,000 \mathrm{rpm}$ for $10 \mathrm{~min}$ at $21^{\circ} \mathrm{C}$.

The upper-layer was extracted from both total lipid and isolated TAG samples, dried under nitrogen gas, and reconstituted in hexane. Samples were analyzed using an Agilent Technologies 7890B gas chromatography system (Agilent Technologies, Mississauga, ON, Canada) with a flame ionization detector, and samples were separated on an Agilent J \& W DBFFAP fused-silica capillary column $(15 \mathrm{~m}, 0.1 \mu \mathrm{m}$ film thickness, $0.1 \mathrm{~mm}$ i.d.; Agilent, Palo Alto, CA, USA). FA peaks were identified by comparison with retention times of FA methyl ester standards. Samples were spiked with $10 \mu \mathrm{l}$ of a $0.1 \mu \mathrm{g} / \mu \mathrm{L}$ C17:0 internal standard and FA abundance was calculated by comparison of individual FA peaks to this standard. To estimate SCD1 and ELOVL6 activity, we used the common product/precursor FA ratio approach (i.e., 18:1n-9/18:0 and 16:1n-7/16:0 for SCD1, and 18:0/16:0 and 18:1n-7/16:1n-7 for ELOVL6) [130, 176, 177]. All FA data was normalized to protein concentrations for each treatment condition. Therefore, FA data is reported as $\mu \mathrm{g}$ FA $/[\mu \mathrm{g} / \mu \mathrm{L}$ protein $] \pm$ SEM.

\subsubsection{RNA and Protein Extraction}

Total RNA and protein were extracted from adipocytes using the Qiagen RNeasy Mini Kit (Qiagen, Mississauga, ON, Canada) and a modified acetone precipitation protocol. Briefly, after spinning samples through an RNeasy spin column, four parts acetone was added to the flow through. The column was retained for standard RNA extraction, and the flow through was 
incubated for $30 \mathrm{~min}$ at $-20^{\circ} \mathrm{C}$ prior to centrifugation at $13,000 \mathrm{rpm}$ for $10 \mathrm{~min}$. The protein pellet was then dried and re-suspended in 10\% sodium dodecyl sulphate (SDS).

Extracted protein was quantified using a detergent-compatible protein assay (BioRad Laboratories, Mississauga, ON, Canada) and extracted RNA was quantified using a Nanodrop (Fisher Scientific, Waltham, MA, USA). Both protein and RNA were stored at $-80^{\circ} \mathrm{C}$ before all analyses. RNA quality was tested using an Agilent 2100 BioAnalyzer (Agilent Technologies Inc., Santa Clara, CA, USA). RNA samples were screened to ensure an RNA integrity number $\geq$ 9 for microarray analyses.

\subsubsection{Western Blot Analysis}

Equal amounts of protein were heat-denatured for $5 \mathrm{~min}$ at $95^{\circ} \mathrm{C}$ and allowed to cool prior to separation by SDS-polyacrylamide gel eletrophoresis on $10 \%$ resolving gels. Proteins were transferred to nitrocellulose membranes at $200 \mathrm{~mA}$ and membranes were subsequently blocked in Tris-buffered saline-0.1\% Tween-20 (TBST) supplemented with either 5\% non-fat dry milk (SCD1, $\alpha$-Tubulin) or 7.5\% BSA (ELOVL6), with gentle agitation for $1 \mathrm{hr}$ at room temperature. Primary antibodies were diluted in TBST/5\% BSA, and membranes were incubated in primary antibody overnight at $4^{\circ} \mathrm{C}$ with gentle agitation. The membranes were then washed two times in TBST. Afterwards, membranes were incubated in TBST/1.0\% non-fat dry milk supplemented with horseradish peroxidase-conjugated secondary antibodies for $1 \mathrm{hr}$ at room temperature. Protein bands were detected using ECL Plus and imaged using a Typhoon Imaging System (GE Health Care, Baie d'Urfe, QC, Canada). Relative band intensities were quantified using Alpha Innotech Software (San Leandro, CA, USA), with $\alpha$-tubulin used as the internal control. 


\subsubsection{Real-Time RT-PCR}

Total RNA $(1 \mu \mathrm{g})$ was used to synthesize single stranded cDNA using a High-Capacity cDNA Reverse Transcription Kit (Life Technologies, Burlington, ON, Canada). cDNA $(0.025 \mu \mathrm{g})$ was subsequently used for real-time RT-PCR using a BioRad CFX96 RT-PCR detection system and SSo FAST EvaGreen Supermix (BioRad Laboratories, Mississauga, ON, Canada), as described previously [64]. All primers were designed using the online Roche Universal Probe Library and Assay Design Center. RPLP0 was used as a housekeeping gene.

\subsubsection{Microarray Analysis}

BioAnalyzer results were used to select four high quality RNA samples per group for microarray analysis. Total RNA (100ng) was used to synthesize cDNA and subsequently cRNA, as described previously [64]. Second cycle cDNA was synthesized, fragmented, biotin labelled, and hybridized to Affymetrix Mouse Gene 1.1 ST array strips (Affymetrix Inc., Fremont, CA, USA) according to the manufacturer's instructions. Array strips were washed, stained, and scanned on the GeneAtlas platform. The microarray data set can be downloaded from the NCBI Gene Expression Omnibus (GSE51905).

\subsubsection{Microarray Cluster Analysis}

Microarray data were imported into R/Bioconductor (http://www. bioconductor.org/) [178, 179] and the robust multi-array method from the oligo package was used to normalize data [180]. An analysis of variance (ANOVA) was used to identify differentially expressed genes. A false discovery rate of 0.05 was used to account for multiple testing. The Parsimonious Gaussian Mixture Models (PGMM) method was used for cluster analysis of differentially expressed genes 
[181-183]. In addition, k-means, k-medoids, and MCLUST clustering methods were used to subdivide larger PGMM clusters in an effort to highlight smaller gene networks [184-188]. Each gene was assigned a four-digit code corresponding to its 'cluster assignment' in all four clustering methods to form robust miniclusters. Only miniclusters comprised of at least 40 genes were considered for subsequent pathway analysis. Transcriptional network analysis (FunNet, http://www.funnet.info) was used to identify biological pathways in both PGMM clusters and miniclusters.

\subsubsection{Statistical Analyses}

A nonparametric Mann-Whitney $U$ test was used to determine statistical significance for gene expression, protein content, lipid abundance, and elongation or desaturation ratios. All data are presented as mean \pm SEM, and a p-value $<0.05$ was considered statistically significant.

\subsection{Results}

\subsubsection{SCD1 Inhibition did not Influence Markers of Adipocyte Differentiation}

We first assessed whether $\mathrm{SCD} 1_{\text {inhib }}$ treatment affected adipocyte differentiation. Gene and protein expression of our primary marker of differentiation, PPAR $\gamma$, was unchanged with $\mathrm{SCD} 1_{\text {inhib }}$ treatment, with the exception of a slight decrease in the expression of the Ppar 2 isoform at day 4 (0.4-fold, $\mathrm{p}<0.05$; Figure 3.1A). However, the decrease in Ppary2 gene expression was not reflected at the protein level for either total Ppary or the Ppary2 isoform. Furthermore, the slight decrease in total PPAR $\gamma$ protein content at day 2 (Figure 3.1B) was not statistically significant. Equivalent results were seen when examining other markers of 
differentiation, for example, FA binding protein 4 (FABP4) and sterol regulatory elementbinding protein-1c (SREBP1c) (data not shown). Together, this led us to conclude that SCD1 $1_{\text {inhib }}$ treatment did not appear to affect adipocyte differentiation.

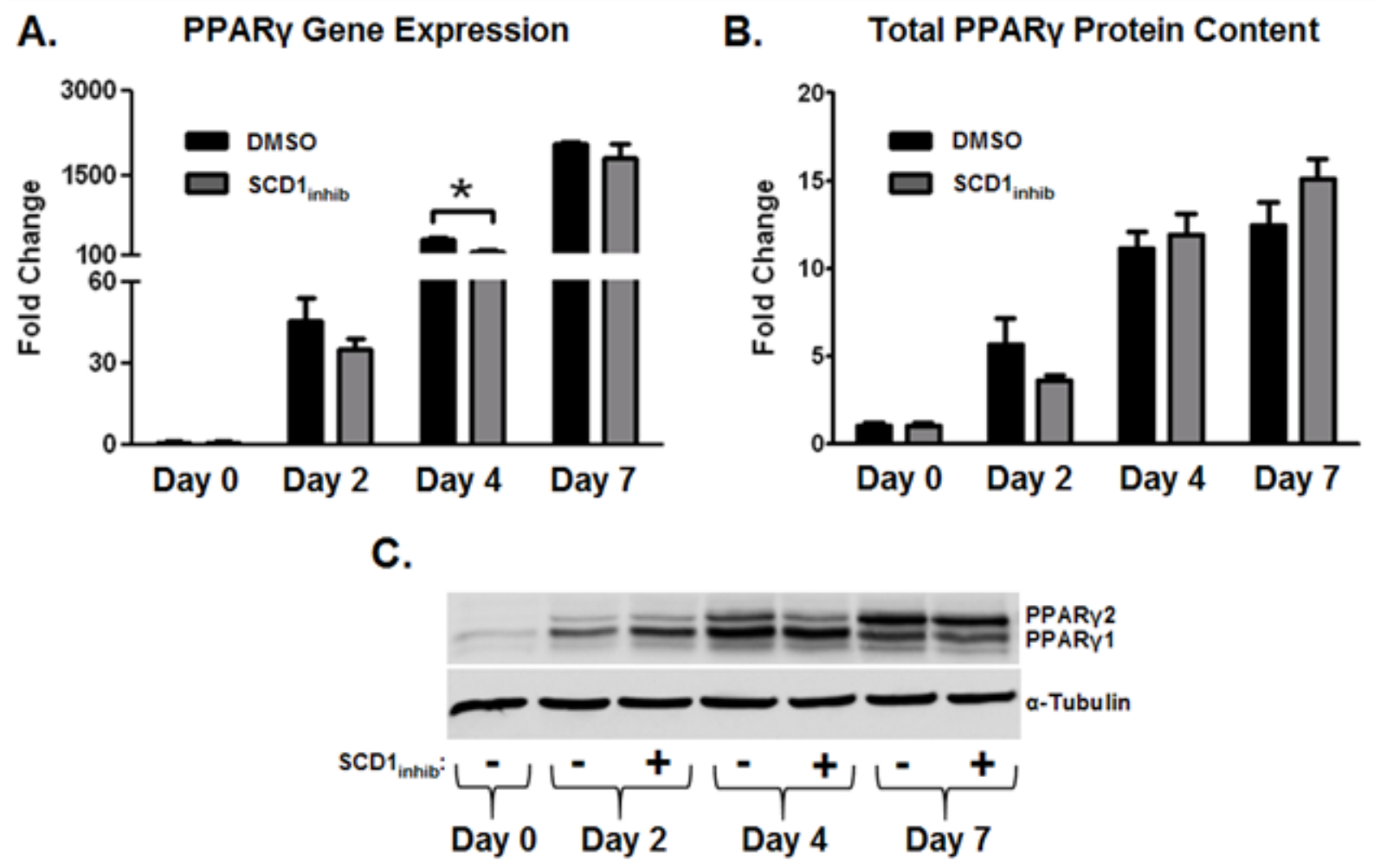

Figure 3.1: Ppary gene expression and protein content in differentiating preadipocytes. (A) Ppary 2 gene expression and (B) total PPAR $\gamma$ protein content at four time points throughout differentiation (days 0,2, 4 and 7) in 3T3-L1 preadipocytes. PPAR $\gamma 1$ and PPAR $\gamma 2$ protein isoforms did not significantly differ throughout differentiation with $\mathrm{SCD} 1_{\text {inhib. }}$. Representative protein blots are shown in $(\mathrm{C})$. Experiments were performed in triplicate $(\mathrm{n}=3)$. *Significant difference between control (DMSO) and $\mathrm{SCD} 1_{\text {inhib }}$ conditions $(* \mathrm{p}<0.05)$.

\subsubsection{Effects of SCD1 Inhibition on SCD and ELOVL6 Expression}

The expression of genes involved in FA biosynthesis (Elovl6, Scd1, and $S c d 2$ ) increased 3.0-fold, 3,000-fold and 30-fold, respectively, during adipocyte differentiation (Figure 3.2). These increases were reflected at the protein level with increases of 4.2-fold and 200 -fold for 
ELOVL6 and SCD1, respectively (Figure 3.3). Importantly, Scd1 gene expression and protein content did not differ between the control and $\mathrm{SCD} 1_{\text {inhib }}$ by day 7 of differentiation; indicating that $\mathrm{SCD} 1_{\text {inhib }}$ disrupts activity rather than $\mathrm{SCD} 1$ gene or protein levels (Figures 3.2 and 3.3). $\mathrm{SCD} 1_{\text {inhib }}$ treatment significantly increased Elovl6 gene expression at day 7 compared with the control (2.8-fold, $\mathrm{p}=0.04$; replicated in quadruplicate) (Figure 3.2). However, the increase in Elovl6 gene expression was not reflected in ELOVL6 protein content at day 7 (1.1-fold, $\mathrm{p}=0.77$ )

(Figure 3.3). Although we observed a slight, statistically significant, decrease in ELOVL6 protein content at day 4 , this difference was lost by day 7 .
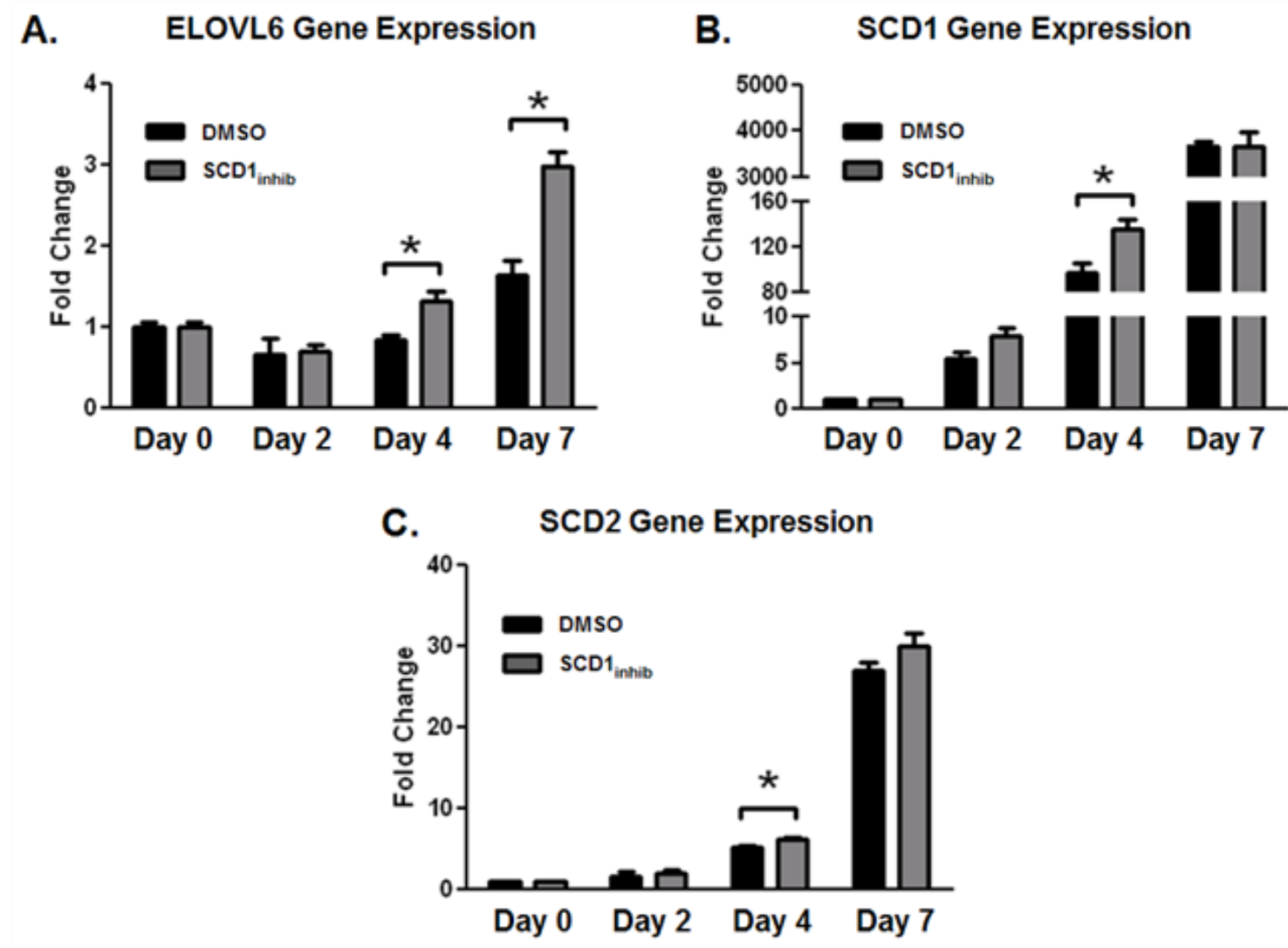

Figure 3.2: Changes in Elovl6, $S c d 1$ and $S c d 2$ gene expression in differentiating preadipocytes. (A) Elovl6, (B) $S c d 1$ and (C) $S c d 2$ gene expression at four time points during differentiation (days 0, 2, 4 and 7) in 3T3-L1 preadipocytes. Experiments were performed in triplicate $(n=3)$. *Significant difference between control (DMSO) and SCD1 $1_{\text {inhib }}$ conditions $(* \mathrm{p}<0.05)$. 
A.

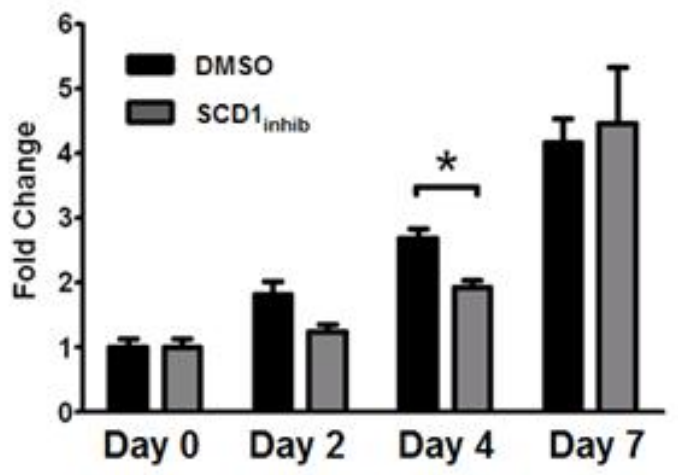

B.

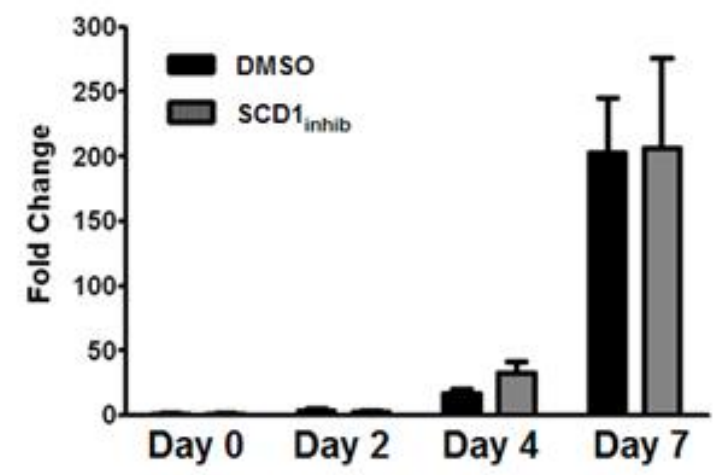

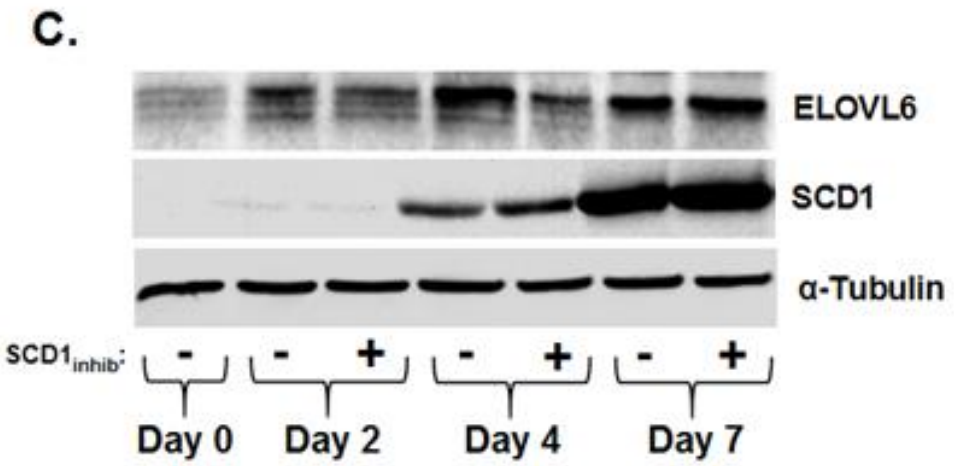

Figure 3.3: Changes in ELOVL6 and SCD1 protein expression in differentiating preadipocytes. (A) ELOVL6 and (B) SCD1 protein content at four time points during differentiation (days 0, 2, 4 and 7) in 3T3-L1 preadipocytes. Experiments were performed in triplicate $(n=3)$. Representative blots are shown in (C). *Significant difference between control (DMSO) and $\mathrm{SCD} 1_{\text {inhib }}$ conditions $(* \mathrm{p}<0.05)$.

\subsubsection{Effects of SCD1 Inhibition on FA Profiles in Differentiated Preadipocytes}

The abundance of 16:0 and 18:0 increased significantly by $45 \%$ and $194 \%$, respectively, with $\mathrm{SCD} 1_{\text {inhib }}$ (Figure 3.4A). In contrast, the abundance of $16: 1 \mathrm{n}-7$ and $18: 1 \mathrm{n}-7$ decreased by $61 \%$ and $35 \%$ with $\mathrm{SCD} 1_{\text {inhib}}$, respectively (Figure 3.4A). Interestingly, the levels of 18:1n-9 increased by $61 \%$ (Figure 3.4A). The observed increase in Elovl6 gene expression, coupled with the robust increase in 18:0 levels (the major ELOVL6 product), lead us to hypothesize that ELOVL6 activity may have been augmented in adipocytes treated with $S C D 1_{\text {inhib. To explore }}$ 
this hypothesis, we estimated ELOVL6 activity using the commonly used product/precursor FA ratio approach [176]. Both the SFA elongation ratio (18:0/16:0) and the MUFA elongation ratio (18:1n-7/16:1n-7) significantly increased with $\mathrm{SCD}_{\text {inhib }}$ (Figure 3.4B). We also estimated SCD activity using $16: 1 \mathrm{n}-7 / 16: 0$ and $18: 1 \mathrm{n}-9 / 18: 0$ ratios $[130,177]$ and found that both $16 \mathrm{C}$ and $18 \mathrm{C}$ SCD ratios were significantly reduced with $S C D 1_{\text {inhib }}$, as expected (Figure $3.4 C$ ).
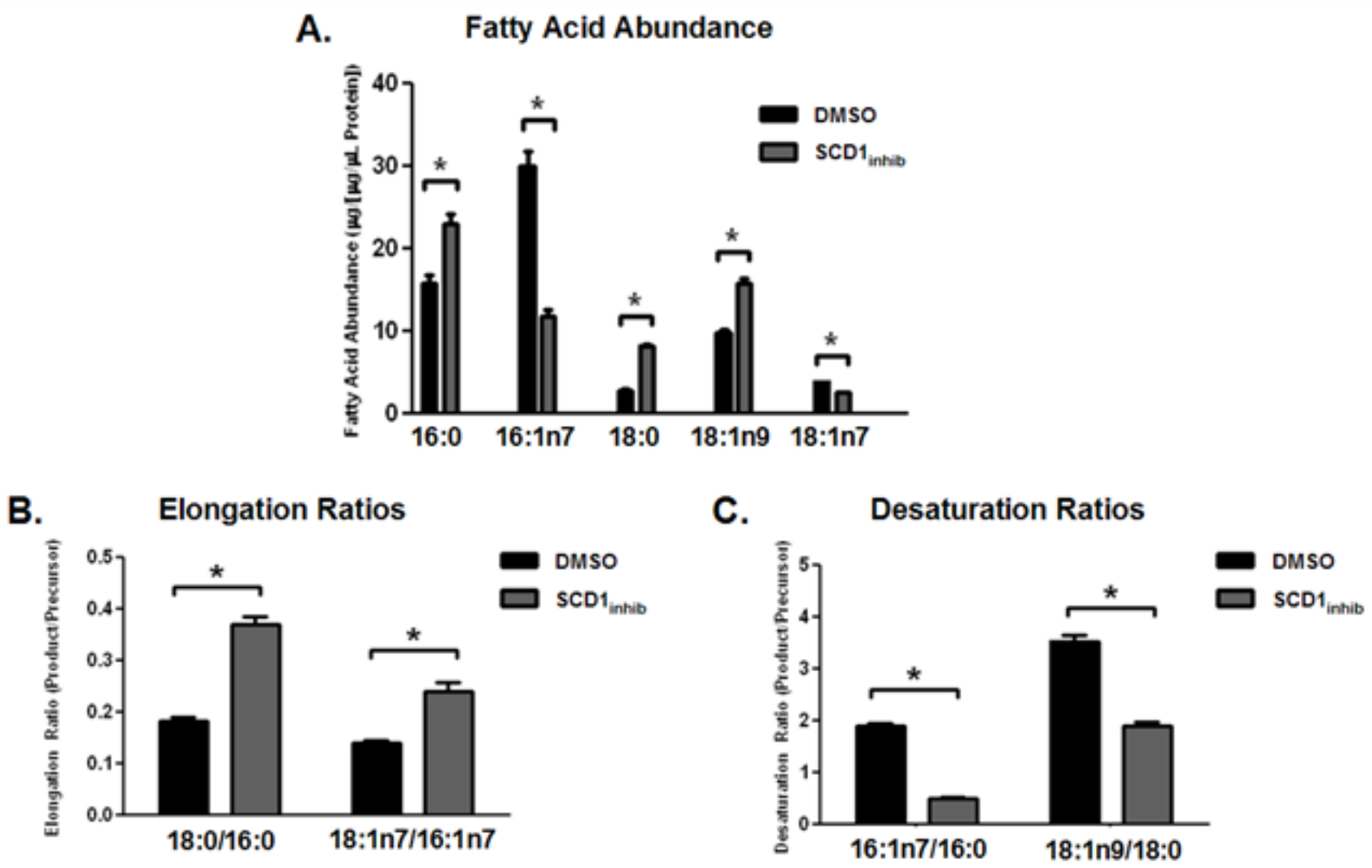

Figure 3.4: FA abundance, desaturation and elongation ratios in differentiated preadipocytes. (A) FA abundance at day 7 in cells treated with either control (DMSO) or SCD1 inhibitor (SCD1 $1_{\text {inhib}}$ ), normalized to protein concentrations for each condition. (B) Elongation ratios and (C) desaturation ratios as estimates of enzymatic activity of ELOVL6 and SCD1, respectively. 16:0, Palmitic acid; 16:1n-7, palmitoleic acid; 18:0, stearic acid; 18:1n-9, oleic acid; 18:1n-7, vaccenic acid. Experiments were performed in quadruplicate $(n=4)$. *Significant difference between control (DMSO) and SCD1 $1_{\text {inhib }}$ conditions $(* \mathrm{p}<0.05)$. 


\subsubsection{The Impact of SCD1 Inhibition on Biological Pathways in Differentiated Preadipocytes}

We assessed global gene expression using microarrays to highlight the genes and pathways underlying cellular adaptations to $S C D 1_{\text {inhib }}$. One of the microarrays in the $S C D 1_{\text {inhib }}$ group was removed from our analysis due to lower array-wide log probe intensities; therefore, our analysis consisted of four control samples and three $\mathrm{SCD} 1_{\text {inhib }}$ samples. 448 genes were significantly upregulated and 564 were downregulated with $\mathrm{SCD} 1_{\text {inhib }}$ (p-value $<0.05$, corrected for multiple testing). Differentially expressed genes were subsequently used for a global functional analysis to highlight the most relevant biological pathways affected with SCD1 inhibition. Seven clusters of co-regulated genes were isolated using the PGMM clustering technique. With the use of additional clustering algorithms, the two larger PGMM clusters (cluster nos. 2 and 6) were further divided into four and three miniclusters, respectively (Figure 3.5). This stringent clustering approach highlighted several biological pathways altered by SCD1 inhibition, including biosynthesis of unsaturated FAs, mitogen-activated protein kinase signaling, and metabolic pathways (Figure 3.5). Genes related to antigen processing and presentation, natural killer cell-mediated cytotoxicity, and JAK-STAT signaling, were upregulated with SCD1 $1_{\text {inhib. }}$ In addition, insulin signalling, FA biosynthesis, glycerolipid and PPAR signaling pathways were all downregulated by $\mathrm{SCD} 1_{\text {inhib }}$ treatment. Genes within the various biological pathways altered by $\mathrm{SCD} 1_{\text {inhib }}$ included Elovl6, which was significantly upregulated. Interestingly, microarray data revealed several genes involved in TAG synthesis were downregulated with $\mathrm{SCD} 1_{\text {inhib}}$, including glucokinase, phosphofructokinase (Pfk), phosphoenolpyruvate carboxykinase $(P c k) 1$ and 2, acetyl-CoA carboxylase alpha $(A c c \alpha)$, diacylglycerol acyltransferase 2 (Dgat2), and PPAR $\gamma$ co-activator-1 $\alpha(\operatorname{Pgcl} \alpha)$. The expression changes for a subset of these genes were subsequently validated by real-time RT-PCR. 
Specifically, glucokinase, $P c k 1, P c k 2, A c c \alpha$ and Dgat2 were downregulated 0.31-fold, 0.11-fold, 0.34-fold, 0.53 -fold and 0.56 -fold by $S C D 1_{\text {inhib }}$ treatment $(\mathrm{p}<0.05$; data not shown). These gene expression changes were validated in two additional independent experiments.

\subsubsection{Adipocyte TAG Abundance and SCD1 inhibition}

As many of the genes identified by microarray analysis suggested that TAG synthesis was downregulated, we next assessed total TAG content in adipocytes treated with SCD1 $1_{\text {inhib. }}$ We observed that total TAG content was significantly reduced by $\sim 33 \%$ in adipocytes treated with $\mathrm{SCD} 1_{\text {inhib }}$ (Figure 3.6). 


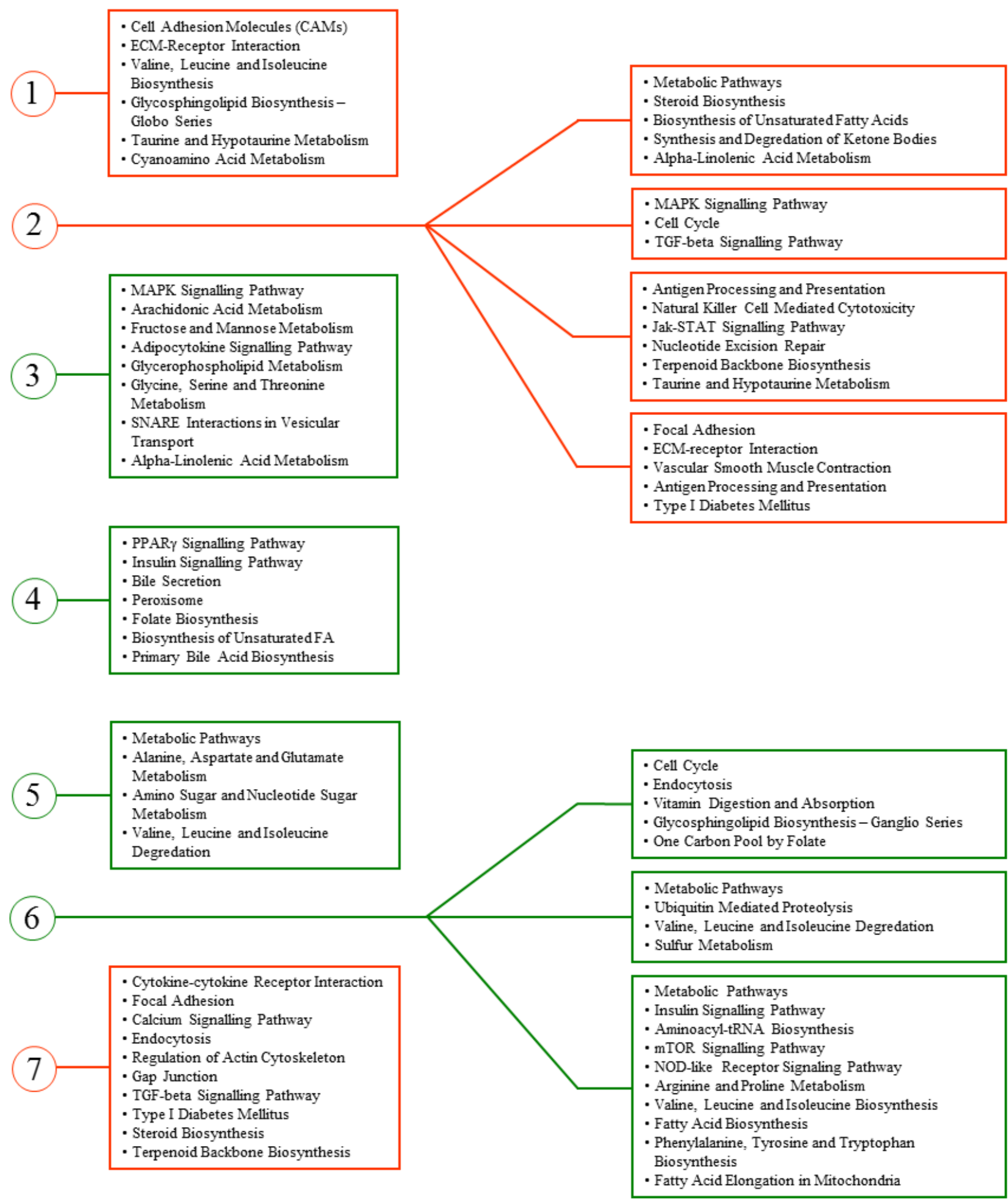

Figure 3.5: Biological pathways altered in differentiated preadipocytes by SCD1 inhibition. Clusters and mini-clusters of KEGG pathways highlighted in microarray data using global functional analyses. Red and green colours indicate the clusters that comprise upregulated and downregulated genes, respectively. For simplicity, only the mini-clusters generated from the two large PGMM clusters (cluster nos. 2 and 6) are depicted. PGMM, Parsimonious Gaussian Mixture Models; KEGG, Kyoto Encyclopedia of Genes and Genome. 


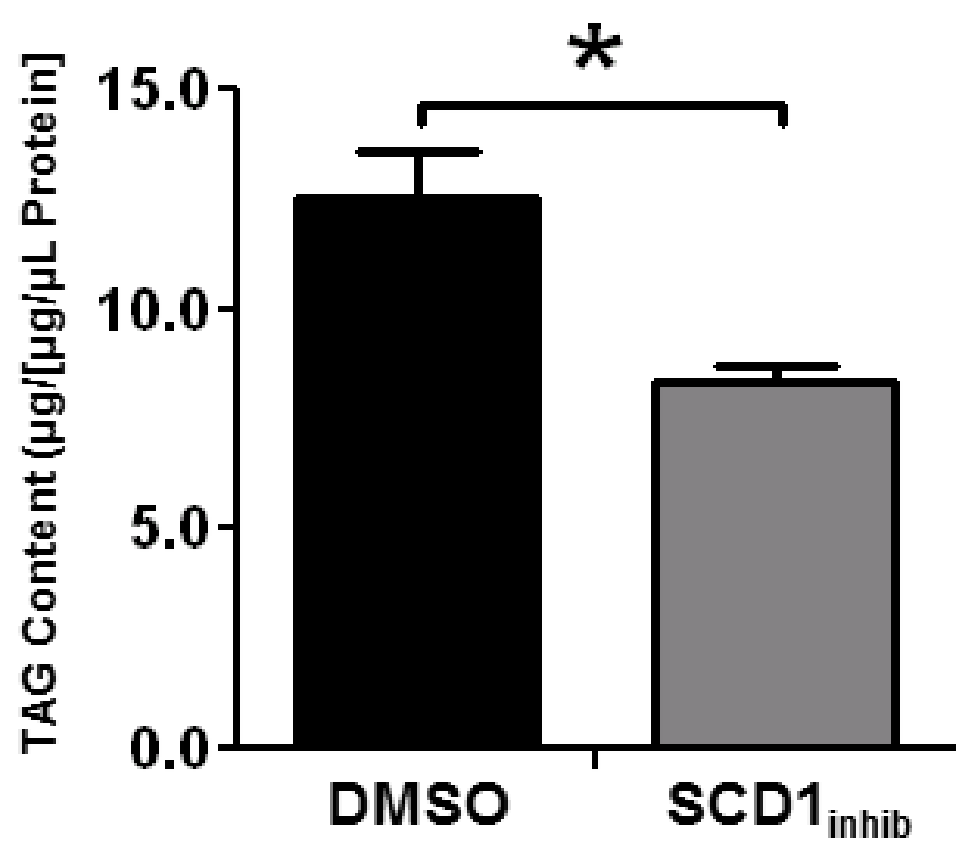

Figure 3.6: Total TAG content in differentiated preadipocytes with control or SCD1 $1_{\text {inhib }}$ treatments. Total TAG content was normalized to protein concentrations for each condition. Experiments were performed in triplicate $(n=3)$. *Significant difference between control (DMSO) and SCD1 $1_{\text {inhib }}$ conditions $(* \mathrm{p}<0.05)$.

\subsection{Discussion}

SCD1 is a critical enzyme involved in de novo lipogenesis. Scdl expression has previously been shown to increase dramatically during adipocyte differentiation [53], and the relationship between SCD1 and obesity is well documented [28, 137, 149, 189]. Specifically, reduced SCD1 activity is associated with improved insulin sensitivity and decreased body weight [137, 149]. Given that SCD1 has previously been positioned as a metabolic hub with a key role in obesity [190], it is important to determine whether reduced SCD1 activity can influence the genetic and molecular processes associated with adipogenesis. Therefore the present 
investigation aimed to examine the effects of SCD1 inhibition on lipid and gene expression profiles in differentiating preadipocytes.

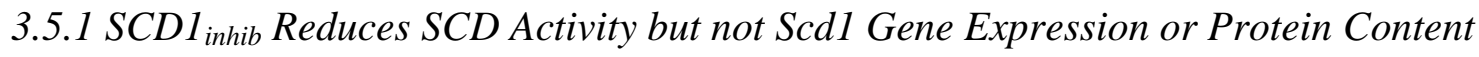

We first set out to ensure that the SCD1 inhibitor used in the current study did not alter adipocyte differentiation. We found no evidence that differentiation was affected with $\mathrm{SCD} 1_{\text {inhib }}$ treatment, as demonstrated by the lack of significant changes in the expression of common markers of differentiation (i.e., Ppary, Fabp4, and Srebp1c). Furthermore, we report that $\mathrm{SCD} 1_{\text {inhib }}$ does not affect $S c d 1$ gene expression or SCD1 protein content in differentiated preadipocytes. However, $\mathrm{SCD} 1_{\text {inhib }}$ led to dramatic decreases in $\mathrm{SCD}$ activity, as estimated using 16:1n-7/16:0 and 18:1n-9/18:0 desaturation ratios. Taken together, this suggests that $\mathrm{SCD} 1_{\text {inhib }}$ blunts SCD1 activity while leaving gene and protein expression unaltered.

\subsubsection{Potential Upregulation of ELOVL6 During SCD1 Inhibition to Prevent 16:0 Accumulation}

SCD1 inhibition had significant effects on gene and protein expression, as well as FA profiles, in differentiated preadipocytes (i.e., day 7). As predicted, $\mathrm{SCD} 1_{\text {inhib }}$ treatment caused an accumulation of SCD1 precursor FAs, that is, 16:0 and 18:0. These findings agree with previous reports in mice lacking SCD1, which showed an accumulation of SFA in various tissues due to the inability to convert SFA into MUFA [28, 191]. The accumulation of SFA can have significant ramifications on metabolic function. For example, evidence suggests that $16: 0$ is a lipotoxic and inflammatory FA that increases proinflammatory gene expression and secretion (e.g., TNFa) via toll-like receptor 4 (TLR4) signalling in adipocytes [191, 192]. As such, SCD1 has an important role regulating the amount of 16:0 in adipocytes. This was reinforced by Collins 
et $a l$., who reported that human adipocytes that were intolerant to 16:0 (i.e., adipocytes that experienced a high degree of cell death when exposed to this FA) had low SCD activity [125]. Furthermore, SCD1 is also required in muscle and pancreatic $\beta$-cells to protect against 16:0induced lipotoxicity [28].

Although we observed a significant increase in the abundance of both 16:0 and 18:0 with SCD1 inhibition, the percentage increase in 18:0 was considerably more substantial than that seen for 16:0 (194\% versus 45\%). Concomitant with changes in SFA abundance was an increase in both Elovl6 gene expression and estimated activity. Taken together, we propose that adipocytes may activate ELOVL6 to convert the more lipotoxic 16:0 into 18:0 when SCD1 function is compromised. In light of previous work from our research group showing that 16:0 and 18:0 trigger distinct transcriptional networks, we believe that up-regulating ELOVL6 activity may offer a degree of protection to adipocytes. For example, we have shown that treating differentiated preadipocytes with 16:0 increased TLR signalling, while 18:0 had no effect on this pathway [64]. In contrast, 18:0 treatment increased PPAR and insulin signaling, whereas 16:0 did not [64]. Considering this, we propose that converting 16:0 into 18:0 via ELOVL6 may serve as a protective adaptation in adipocytes when SCD1 activity is inhibited to minimize the accumulation of cellular 16:0. This hypothesis is supported by previous work by Collins et al., who reported that SCD1 and ELOVL6 gene expression, and de novo lipogenesis, were upregulated in human adipocytes treated with 16:0 to minimize the lipotoxic effects of 16:0 [125]. Taken together, our results support the notion that SCD1 activity should be maintained within a normal range to ensure healthy cell function [193]. 


\subsubsection{Altered MUFA Content in SCD1-Inhibited Adipocytes}

When differentiating adipocytes were treated with $\mathrm{SCD} 1_{\text {inhib}}$, we observed significant decreases in the abundance of 16:1n-7 and 18:1n-7, and an increase in 18:1n-9. It was previously suggested that 16:1n-7 and its metabolites may act as lipid signalling molecules (i.e., lipokines) secreted from adipocytes or AT in mice that may offer protection from diet-induced obesity and improve whole-body glucose metabolism [13, 164]. The reduction in 16:1n-7 levels in adipocytes with reduced SCD1 activity may provide a potential mechanism to explain the modifications in whole-body metabolism seen in Scd1-/- mice. The marked decrease in 16:1n-7 seen with $\mathrm{SCD} 1_{\text {inhib }}$ also provides an explanation for the reduction in 18:1n-7. Specifically, the lower abundance of 16:1n-7 means less will be elongated to 18:1n-7 by ELOVL6. Furthermore, the preferred substrate for ELOVL6 is 16:0, not 16:1n-7 [194]. Taken together, this provides a plausible explanation for the lower levels of 18:1n-7 seen with reduced SCD1 activity. Although decreases in $16: 1 \mathrm{n}-7$ and $18: 1 \mathrm{n}-7$ were expected, the increase in $18: \ln 9$ abundance was unexpected. Although $\mathrm{SCD} 1_{\text {inhib }}$ treatment significantly reduced the 18:1n-9/18:0 desaturation ratio, our results show that desaturation activity was not completely abolished (Figure 3.4). Therefore, the increase in 18:1n-9 most likely stems from increased 18:0 production (via ELOVL6) and the subsequent desaturation by residual SCD1 activity; something that can be specifically tested in future studies using radiolabelled tracers. However, we acknowledge that we cannot rule out the possible contribution of SCD2 to the increased levels of 18:1n-9.

\subsubsection{SCD1 Inhibition Alters Metabolic Pathways and Reduces TAG Synthesis}

We used a global gene expression approach as a means to determine which biological pathways may be underlying the cellular adaptations observed with SCD1 inhibition. Clustering 
microarray data revealed that the insulin signaling pathway was downregulated with SCD1 ${ }_{\text {inhib. }}$. This finding is intriguing given previous evidence that $S c d 1-/-$ mice have improvements in insulin sensitivity $[159,195]$. However, it is now recognized that multiple metabolic tissues are involved in the improvements and adaptations seen in Scd1-/- mice [28]. Further, tissue-specific Scd1-/- mouse models have often shown effects that differ from that seen in Scd1-/- mice [23, 27]. For example, Hyun et al. showed an increase in glucose transporter 1 (GLUT1) expression, but not GLUT4, in AT-specific $S c d 1$ knockout (AKO) mice compared with $S c d 1$-/- mice [27]. In addition, these same authors treated 3T3-L1 adipocytes with an SCD1 inhibitor (different from that used in the current study) and showed that insulin signaling and insulin-stimulated glucose uptake were unaffected in adipocytes [27]. In contrast, Collins et al. reported reduced insulin sensitivity in human adipocytes lacking SCD1 [125]. Altogether, this suggests that the improvements in insulin sensitivity seen in $S c d 1$-/- mice may not stem from adipocytes or AT.

Alongside decreases in the expression of genes involved in glycerolipid and biosynthesis of unsaturated FA pathways, we observed a significant decrease in total TAG content in adipocytes treated with $\mathrm{SCD} 1_{\text {inhib. }} \mathrm{SCD} 1$-deficient mice were previously reported to have reduced hepatic TAG synthesis [11]. One mechanism proposed to explain this decrease in hepatic TAG synthesis was the close proximity of SCD1 with diacylglycerol acyltransferase 2 (DGAT2) in the endoplasmic reticulum [32, 101, 196]. Specifically, SCD1 was postulated to produce MUFA within the vicinity of DGAT2 to facilitate their incorporation into TAG [10]. Two DGAT isoforms (DGAT1 and DGAT2) exist in AT and together they produce nearly all of the endogenously synthesized TAG in murine adipocytes [103]. It has been suggested that hepatic DGAT2 incorporates de novo synthesized FAs into TAG whereas DGAT1 acts downstream of DGAT2, using exogenous FAs and partial glyceride products [104]. These 
findings, coupled with the notion that MUFA produced by SCD1 may be the main substrates for TAG synthesis [10, 17, 101], are supported by our observations of decreased Dgat2 gene expression and total TAG content. This aligns with a previous study reporting that de novo lipogenesis was reduced in human adipocytes lacking a functional SCD1 [125].

\subsubsection{Proposed Model for Reduced TAG Content in SCD1-Inhibited Adipocytes}

On the basis of our gene expression data, we have identified a coordinated response in the expression of numerous genes that further contribute to the reduced TAG content seen with SCD1 inhibition (Figure 3.7). First, the decreased expression of glucokinase, Pfk, Pckl, and Pck2 seen with SCD1 inhibition can reduce dihydroxyacetone phosphate (DHAP) production. DHAP is the precursor for synthesizing glycerol-3-phosphate (glycerol-3-P), which is required for FA (re-)esterification into TAG. Second, decreased Dgat2 can also contribute to reduced TAG content, as outlined above. Finally, decreased Acc $\alpha$ expression can reduce the synthesis of endogenous FAs used for TAG production. Taken together, the modifications in gene expression seen with reduced SCD1 activity have the potential to coordinately decrease glycerol-3-P and FA synthesis, which in turn could reduce TAG abundance in adipocytes. 


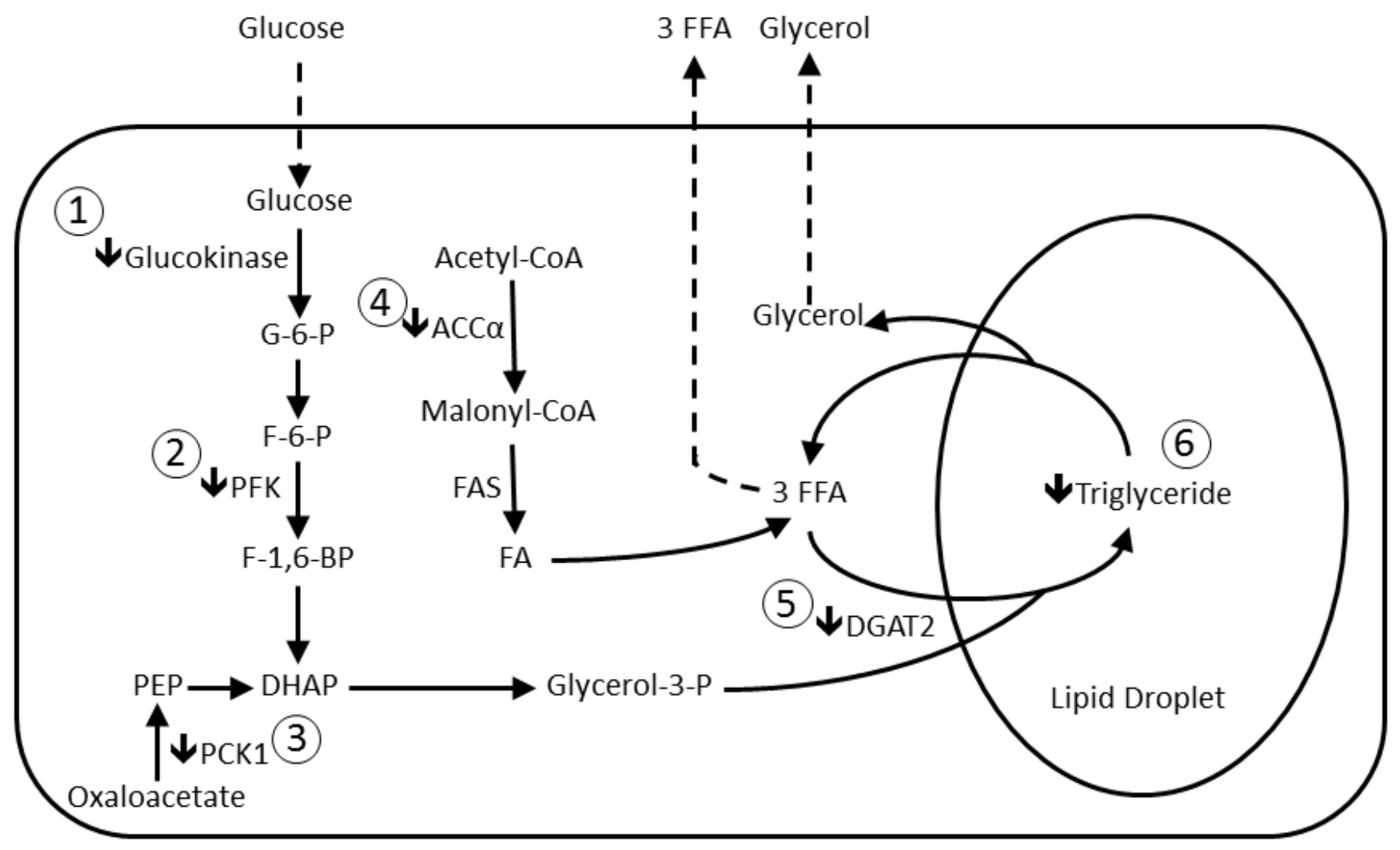

Figure 3.7: Proposed mechanism by which SCD1 inhibition reduces total TAG in differentiated preadipocytes. (1), (2) and (3) indicate genes whose expression changed with SCD1 inhibition and would decrease the production of DHAP. This hinders the production of glycerol-3-P, which is needed for TAG synthesis from FA (re-)esterification. (4) Decreased Acc $\alpha$ hinders endogenous FA synthesis necessary for TAG synthesis. These aforementioned adaptations to SCD1 inhibition, as well as a significant decrease in Dgat2 expression (5), would ultimately lead to decreased TAG synthesis in differentiated preadipocytes (6). ACC $\alpha$, acetyl-CoA carboxylase alpha; DGAT2, diacylglycerol acyltransferase 2; DHAP, dihydroxyacetone phosphate; FAS, fatty acid synthase; FFA, free fatty acid; F-1,6-BP, fructose-1,6-bisphosphate; F-6-P, fructose-6phosphate; G-6-P, glucose-6-phosphate; PCK1, phosphoenolpyruvate carboxykinase 1; PFK, phosphofructokinase. 
There are some limitations to consider with the current study. First, adipocytes are not the only cell type in AT. Indeed, AT is also composed of a stroma vascular fraction that is comprised of macrophages, endothelial cells, mesenchymal stem cells, etc. [140]. Future investigations could consider using AT explants to explore the effects of SCD1 inhibition on ELOVL6 activity and TAG synthesis. Second, although the product/precursor ratio is commonly used to estimate enzymatic activity from FA data, we propose that tracer experiments using radiolabelled 16:0 represent the logical next step to further study how reduced SCD1 activity affects FA elongation in adipocytes.

\subsubsection{Summary and Perspectives}

We investigated the effects of reduced SCD1 activity on gene expression and FA profiles in differentiating preadipocytes. SCD1 inhibition did not appear to affect adipocyte differentiation. In contrast, SCD1 inhibition resulted in an altered FA profile, with increases in 16:0, 18:0, and 18:1n9, and concomitant decreases in 16:1n-7 and 18:1n-7. Furthermore, the notable $194 \%$ increase in 18:0 abundance suggests that SCD1 inhibition in adipocytes triggers a compensatory mechanism by which ELOVL6 is activated to minimize cellular increases in the lipotoxic 16:0. We also found that biological pathways and genes associated with TAG synthesis were downregulated with SCD1 inhibition, providing a plausible molecular mechanism to explain the observed decreases in adipocyte TAG content. As such, our results suggest that when SCD1 activity is reduced, adipocytes upregulate ELOVL6 and downregulate TAG synthesis, likely in an attempt to mitigate lipotoxicity and downstream metabolic perturbations. Ultimately, our data demonstrates that a reduction in SCD1 activity has profound effects on adipocyte 
metabolism, and reinforces that regulating SCD1 activity could be an avenue to reduce the risk of obesity-related complications. 


\section{CHAPTER 4:}

SCD1 INHIBITION DURING 3T3-L1 ADIPOCYTE DIFFERENTIATION REMODELS TRIACYLGLYCEROL, DIACYLGLYCEROL AND PHOSPHOLIPID FATTY ACID COMPOSITION

As published in Prostaglandins Leukotrienes and Essential Fatty Acids (PLEFA):

Jessica C. Ralston and David M. Mutch (2015). SCD1 inhibition during 3T3-L1 adipocyte differentiation remodels triacylglycerol, diacylglycerol and phospholipid fatty acid composition. Prostaglandins Leukot Essent Fatty Acids. Epub Apr 282015. 


\subsection{Abstract}

The conversion of saturated fatty acids (FAs) palmitate (16:0) and stearate (18:0) into monounsaturated FAs palmitoleate (16:1n-7) and oleate (18:1n-9) is catalyzed by stearoyl-CoA desaturase 1 (SCD1). These FAs represent the dominant constituents of adipocyte triacylglycerols (TAGs) and phospholipids (PLs). Given the critical role of SCD1 in lipid metabolism and the notable increase in its expression during adipogenesis, reductions in SCD1 activity have the potential to compromise the adipocyte's ability to accumulate lipid. The current study used thin-layer and gas chromatography to examine the content and FA composition of TAGs, PLs, cholesteryl esters, diacylglycerols and free fatty acids in SCD1-inhibited differentiating 3T3-L1 adipocyte cells. SCD1 inhibition reduced total cellular PL and TAG content concurrent with the down-regulation of genes involved in TAG and PL biosynthesis; however, the relative amount of PL was unaltered. While total DAG levels were increased 2.7fold in SCD1-inhibited adipocytes, this did not induce JNK activation; however, phosphorylated (Ser473) AKT was significantly reduced. As expected, total SFA and MUFA content were increased ( 1.3-fold) and decreased ( 4.0-fold). Further, SCD1 inhibition caused a $\sim 2.2$-fold increase and a $\sim 8.3$-fold decrease in total cellular 18:0 and 16:1n-7 levels, respectively. Similar changes were also seen in other lipid fractions. The levels of other FAs, including polyunsaturated FAs, were also changed in SCD1-inhibited adipocytes. Together, these results add to the growing body of knowledge regarding SCD1 function in adipocytes and highlight its important role in regulating global adipocyte lipid composition. 


\subsection{Introduction}

Understanding the function and development of adipose tissue (AT) is important given the global increase in the prevalence of obesity and obesity-related complications [5, 197]. AT, and adipocytes in particular, have a central role in the regulation of whole-body energy homeostasis by storing excess energy as triacylglycerol (TAG) and secreting adipokines that have paracrine and endocrine functions [5, 197, 198]. The development of AT involves differentiation of preadipocytes into mature lipid-filled adipocytes (i.e., a process referred to as adipogenesis) $[5,6]$. This process is imperative for storing excess lipid in adipocytes and therefore plays an important role preventing the downstream metabolic complications associated with ectopic lipid deposition. During adipogenesis a transcriptional network is activated to stop cellular proliferation and promote the production and uptake of lipid for storage as TAG [7, 199]. One of the key enzymes that facilitates lipid storage is stearoyl-CoA desaturase 1 (SCD1), which catalyzes the conversion of the saturated fatty acids (SFAs) palmitate (16:0) and stearate (18:0) into the monounsaturated fatty acids (MUFAs) palmitoleate (16:1n-7) and oleate (18:1n-9), respectively [28].

Reduced whole-body SCD1 activity is associated with improved insulin sensitivity, decreased body weight, and changes in lipid metabolism [28, 137]. Specifically, Scd1-/- mice are protected against diet-induced obesity through reduced TAG and fatty acid (FA) synthesis [26, 28]. Further, Scd1-/- mice have enhanced FA oxidation in numerous metabolic tissues (e.g., brown AT, muscle and liver), and increased insulin sensitivity in white AT [28, 200]. In contrast, studies focusing on SCD1 in AT have shown that an adipose-specific deficiency in SCD1 (as seen in AKO mice) does not protect from obesity or affect glucose tolerance in mice [160]. 
Additionally, we previously demonstrated that inhibiting SCD1 in differentiated adipocytes down-regulated genes involved in TAG biosynthesis and, ultimately, reduced total TAG content [201]. Taken together, these results emphasize the need for continued investigations into the role of SCD1 and the consequences of altered SCD1 activity in adipocytes.

Previous research has shown that MUFA produced by SCD1 (i.e., 16:1n-7 and 18:1n-9) are the predominant constituents of membrane phospholipids (PLs), cholesteryl esters (CEs), and TAG in 3T3-L1 adipocytes, as well as murine and human AT [11, 40, 66, 202]. While the influence of reduced SCD1 expression on the content of various lipid fractions (i.e., PL, TAG, $\mathrm{CE}$, free fatty acids (FFA) and diacylglycerol (DAG)) is well documented in hepatocytes and liver $[11,73,124,203]$, the effects in adipocytes and AT are not fully delineated. However, SCD1 is highly relevant to study in adipocytes given that this enzyme has a central role regulating cellular levels of SFAs and MUFAs, which consequently has implications on the FA composition of lipid fractions as well as numerous signalling pathways (e.g., insulin signalling) [204].

The objective of the present investigation was to examine the impact of SCD1 inhibition on the content of lipid fractions (i.e., PL, TAG, CE, FFA and DAG) in differentiating adipocytes, as well as FA classes (i.e., SFA, MUFA, and PUFA) within each lipid fraction. Furthermore, we conducted a comprehensive analysis of the distinct FAs within each lipid fraction. Finally, we also examined several key signalling proteins that are known to be influenced by changes in cellular lipids. Together, this research provides new insights into the critical role of SCD1 as a regulator of adipocyte function and lipid metabolism. 


\subsection{Materials and Methods}

\subsubsection{Chemicals and Cell Culture Reagents}

Murine 3T3-L1 preadipocytes were purchased from ATCC (Rockville, MD, USA). Cell culture reagents including Dulbecco's modified Eagle's medium (DMEM), 0.25\% trypsinethylenediamine tetra-acetic acid solution, and penicillin-streptomycin (pen-strep) were purchased from Hyclone laboratories (Logan, UT, USA). The SCD1-specific inhibitor (CAY10566) was purchased from Cayman Chemical (Ann Arbor, MI, USA). All other cell culture reagents including 3-isobutyl-1-methylxanthine (IMBX), dexamethasone (Dex), human insulin, fetal bovine serum (FBS), and dimethylsulfoxide (DMSO; $\geq 99.9 \%$ purity) were purchased from Sigma Aldrich (St. Louis, MO, USA). The following primary antibodies were purchased from Cell Signaling Technology (Danvers, MA, USA): p-JNK, JNK, p-AKT ${ }^{\mathrm{Ser} 473}$, p$\mathrm{AKT}^{\mathrm{Thr} 308}$, and AKT2. The $\alpha$-tubulin antibody was purchased from Abcam (Toronto, ON, Canada).

\subsubsection{Cell Culture Experiments}

A stock solution of the $\mathrm{SCD} 1$ inhibitor ( $\mathrm{SCD} 1_{\text {inhib}}$ ) was created by diluting the inhibitor to $10 \mu \mathrm{M}$ in DMSO. This stock solution was subsequently diluted in media to a final working concentration of $10 \mathrm{nM} \mathrm{SCD} 1_{\text {inhib }}$ for all adipocyte experiments. Adipocytes treated with this dose of $\mathrm{SCD} 1_{\text {inhib }}$ showed $\sim 51 \%$ inhibition of SCD1 activity during adipogenesis, where the desaturation index (i.e., the sum of SCD1 MUFA products divided by the sum of SCD1 SFA substrates) was used to estimate activity. 
3T3-L1 adipocytes were cultured in basic media consisting of DMEM supplemented with $1 \%$ pen-strep and $5 \%$ heat-inactivated FBS. Cells were maintained in culture at $5 \% \mathrm{CO}_{2}, 100 \%$ humidity, and $37^{\circ} \mathrm{C}$ for the duration of all experiments. Adipocyte differentiation was carried out as described previously [201]. Briefly, differentiation was induced at day 0 (i.e., 2 days post confluence) with a standardized differentiation cocktail consisting of IBMX (0.5 mM), Dex (1 $\mu \mathrm{M})$, and human insulin $(5 \mu \mathrm{g} / \mathrm{ml})$ in basic media. After 2 days (i.e., day 2) media was replaced with maintenance media consisting of basic media supplemented with only human insulin (5 $\mu \mathrm{g} / \mathrm{ml})$. Maintenance media was changed every two days until the day 7 endpoint. Throughout differentiation, cells were also treated with either $10 \mathrm{nM} \mathrm{SCD1} 1_{\text {inhib }}$ or an equivalent volume of DMSO (i.e., the control condition). All experiments were conducted with technical replicates in three different passages to ensure that results were not due to passage number.

\subsubsection{Lipid Extraction and Quantification}

Lipid was extracted at four time points during differentiation (days 0, 2, 4 and 7) using a modified Bligh and Dyer method that has been described previously [173, 174, 201]. At each extraction time point, culture media was removed and cells were washed with 1X PBS. Cells were then trypsinized for $5 \mathrm{~min}$ before being collected and centrifuged at $290 \mathrm{rcf}$ for $5 \mathrm{~min}$ to form a cell pellet. The supernatant was removed and the cell pellet was washed in 1X PBS. Next, a methanol:chloroform solution $(2: 1, \mathrm{v} / \mathrm{v})$ was added, and samples were vortexed for 5 min to break up cell pellets. A chloroform:water solution $(1: 1, \mathrm{v} / \mathrm{v})$ was then added prior to centrifugation at $1250 \mathrm{rcf}$ for $20 \mathrm{~min}$. The bottom organic phase was collected and dried under a gentle stream of nitrogen gas. 
Samples were reconstituted in $100 \mu \mathrm{L}$ of chloroform and spotted on Silica-G thin-layer chromatography (TLC) plates, as previously described [205]. Standards for each fraction were purchased from Avanti Polar Lipids Incorporated (Alabaster, AL, USA), and were spotted in a separate TLC lane (i.e., $25 \mu \mathrm{L}$ of PL, TAG, DAG, CE and FFA) [205]. Plates were then placed in a 20x5 cm TLC chamber containing petroleum ether, ethyl ether, and acetic acid (80:20:1, v/v/v), and run for 45 min. PL, TAG, DAG, CE and FFA bands were visualized with $0.1 \%$ 8anilino-1-naphthalene sulfonic acid. Bands were scraped into clean glass vials, and then hexane and $14 \% \mathrm{BF}_{3}-\mathrm{MeOH}(1: 1, \mathrm{v} / \mathrm{v})$ were added before methylation for $1.5 \mathrm{hrs}$ at $100^{\circ} \mathrm{C}$. Samples were cooled, double-distilled water was added, and samples were then centrifuged at $21^{\circ} \mathrm{C}$ for 10 min at 200 rcf. The upper-layer was collected, dried under nitrogen gas, and samples were reconstituted in hexane.

Samples were analyzed using an Agilent Technologies 7890B gas chromatography system (Agilent Technologies, Mississauga, ON, Canada) with a flame ionization detector and separated on an Agilent J\&W DB-FFAP fused-silica capillary column $(15 \mathrm{~m}, 0.1 \mu \mathrm{m}$ film thickness, $0.1 \mathrm{~mm}$ i.d.; Agilent, Palo Alto, CA, USA). FA methyl ester standard peaks were used to identify all FA peaks. Samples were spiked with $1 \mu \mathrm{g}$ C17:0 internal standard and individual FA peaks were compared to this standard for quantification. Total cellular lipid content was calculated by summing FAs measured across all lipid fractions. Similarly, total cellular content for a specific FA (e.g., total cellular 18:0) was calculated by summing relevant values from each lipid fraction. FA data was normalized by cell count for each treatment condition. FA data is reported as $\mu \mathrm{g}$ FA $/ 1 \times 10^{6}$ cells \pm SEM. 


\subsubsection{Gene Expression Analyses}

Total RNA was extracted and used to synthesize single-stranded cDNA for amplification on a Bio-Rad CFX96 RT-PCR detection system (Bio-Rad Laboratories, Mississauga, ON, Canada), as previously described [64]. Primers for acylglycerol-3-phosphate acyltransferase 3 (Agpat3), Agpat9, and diacylglycerol kinase delta $(D g k \delta)$ were designed online using the Roche Universal Probe Library and Assay Design Center, and RplpO was used as the housekeeping gene. Changes in gene expression were determined using the $\Delta \Delta \mathrm{CT}$ normalization and quantification method [206].

\subsubsection{Western Blot Analyses}

Protein was extracted and analyzed as previously described [201]. Briefly, equal amounts of protein were separated on $10 \%$ gels to assess the protein content of p-JNK, JNK, p-AKT ${ }^{\text {Ser473 }}$,

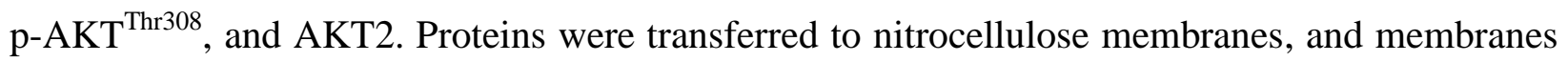
were then blocked for $1 \mathrm{hr}$ with gentle agitation in Tris-buffered saline- $0.1 \%$ Tween-20 (TBST) supplemented with either $5 \%$ non-fat dry milk or $5 \%$ bovine serum albumin (BSA). Membranes were incubated overnight at $4^{\circ} \mathrm{C}$ with primary antibodies in TBST supplemented with BSA. Specific primary antibody dilutions were as follows: p-JNK - 1:500; JNK, p-AKT ${ }^{\text {Thr308, }}$ $\mathrm{AKT}^{\mathrm{Ser} 473}$ and AKT2- 1:1000, and $\alpha$-tubulin - 1:5000. Membranes were washed the following day in TBST and incubated for $1 \mathrm{hr}$ with TBST-1\% non-fat dry milk supplemented with horseradish peroxidase conjugated donkey anti-rabbit or goat anti-mouse secondary antibody at a 1:2000 dilution. Protein bands were detected using ECL Plus and quantified using Alpha Innotech Software (San Leandro, CA, USA). 


\subsubsection{Statistical Analyses}

The effects of time (i.e., differentiation) and treatment (i.e., DMSO vs. SCD $1_{\text {inhib }}$ ) were assessed using a two-way ANOVA. Post-hoc analyses were performed using a Fisher's Least Significant Different test in order to examine differences between DMSO and SCD1 $1_{\text {inhib }}$ conditions at specific time points during differentiation. A p-value $<0.05$ was considered statistically significant. All data are presented as mean \pm SEM.

\subsection{Results}

\subsubsection{Effects of SCD1 Inhibition on Total Cellular Lipid Content Throughout Adipogenesis}

We first examined the effects of SCD1 inhibition on total cellular lipid content, as well as the levels of individual lipid fractions, i.e., PL, TAG, CE, FFA, and DAG (Table 4.1). In control adipocytes, total cellular lipid content increased significantly between days 4 and 7 ( 2.6-fold; $\mathrm{p}<0.001$ ); however, this increase was partially blunted in adipocytes treated with $\mathrm{SCD} 1_{\text {inhib }}$ ( 1.9-fold; $\mathrm{p}=0.001)$. The reduction in total cellular lipid content observed with SCD1 inhibition stemmed from significant decreases in total PL and TAG levels (Table 4.1). While there was a trend for $\mathrm{SCD} 1_{\text {inhib }}$ to increase total $\mathrm{CE}$ and FFA levels during differentiation, post-hoc testing indicated that this only differed significantly from control cells at day 7 (Table 4.1). Additionally, $\mathrm{SCD} 1_{\text {inhib }}$ caused a significant increase in total DAG at both day $4(\sim 1.8$-fold; $\mathrm{p}=0.02)$ and day $7(\sim 2.7$-fold; $\mathrm{p}<0.001)$ compared to control cells (Table 4.1).

When examining the individual lipid fractions as a relative percentage of total lipid at the day 7 endpoint, we also observed a significant decrease in the relative proportions of cellular TAGs and significant increases in the relative proportions of DAGs, CEs, and FFAs (Figure 4.1). 
In contrast, $\mathrm{SCD} 1_{\text {inhib }}$ treatment had no effect on the relative proportion of cellular PLs. In order to determine more specific effects of SCD1 inhibition on adipocyte lipid metabolism, we next examined FA classes (i.e., total cellular SFA, MUFA and PUFA), as well as the content of individual FAs within each lipid fraction. 
Table 4.1

\begin{tabular}{|c|c|c|c|c|c|c|c|c|c|c|}
\hline \multirow{3}{*}{ Lipid Fraction } & \multicolumn{7}{|c|}{ Total Fatty Acid Content $\left(\mu \mathrm{g} / 1 \times 10^{6}\right.$ cells \pm SEM) } & \multirow{3}{*}{ p-Time } & \multirow{3}{*}{$p-T x$} & \multirow{3}{*}{ p-Int } \\
\hline & \multirow[t]{2}{*}{ Day 0} & \multicolumn{2}{|c|}{ Day 2} & \multicolumn{2}{|c|}{ Day 4} & \multicolumn{2}{|c|}{ Day 7} & & & \\
\hline & & DMSO & SCD1 $1_{\text {inhib }}$ & DMSO & SCD1 $1_{\text {inhib }}$ & DMSO & SCD1 $1_{\text {inhib }}$ & & & \\
\hline Phospholipids & $91.13 \pm 10.87$ & $90.94 \pm 11.68$ & $93.04 \pm 5.39$ & $65.34 \pm 2.58$ & $64.13 \pm 3.57$ & $98.25 \pm 5.16$ & $68.20 \pm 2.82^{*}$ & 0.0030 & 0.1795 & 0.1242 \\
\hline Triacylglycerols & $3.32 \pm 0.48$ & $3.21 \pm 0.32$ & $3.15 \pm 0.20$ & $17.26 \pm 2.27$ & $10.47 \pm 0.81$ & $112.45 \pm 14.11$ & $43.77 \pm 3.41^{*}$ & $<0.0001$ & $<0.0001$ & $<0.0001$ \\
\hline Cholesteryl Esters & $1.46 \pm 0.18$ & $0.87 \pm 0.08$ & $1.09 \pm 0.06$ & $0.84 \pm 0.15$ & $1.29 \pm 0.27$ & $2.71 \pm 0.43$ & $5.54 \pm 1.67^{\star}$ & $<0.0001$ & 0.0545 & 0.1012 \\
\hline Free Fatty Acids & $19.89 \pm 2.47$ & $12.34 \pm 2.28$ & $13.48 \pm 1.11$ & $10.3 \pm 0.95$ & $10.71 \pm 0.73$ & $26.64 \pm 4.34$ & $40.89 \pm 8.72^{*}$ & $<0.0001$ & 0.1944 & 0.2689 \\
\hline Diacylglycerols & $1.01 \pm 0.12$ & $0.62 \pm 0.06$ & $0.66 \pm 0.04$ & $0.84 \pm 0.05$ & $1.54 \pm 0.15^{\star}$ & $1.44 \pm 0.11$ & $3.89 \pm 0.32^{*}$ & $<0.0001$ & $<0.0001$ & $<0.0001$ \\
\hline Total & $115.92 \pm 12.99$ & $105.72 \pm 13.12$ & $111.20 \pm 6.00$ & $92.59 \pm 6.22$ & $83.79 \pm 5.32$ & $236.33 \pm 21.19$ & $161.00 \pm 15.99^{*}$ & $<0.0001$ & 0.0363 & 0.0103 \\
\hline
\end{tabular}

Total Fatty Acid Content of Lipid Fractions. Total cellular lipid content and individual lipid fractions in adipocytes treated with or without $\mathrm{SCD} 1_{\text {inhib. }}$ A two-way ANOVA was used to examine the effects of time (p-Time), treatment (p-Tx) and time $\times$ treatment interactions (p-Int). *Indicates a significant difference by Fisher's Least Significant Difference post-hoc test ( $<0.05)$.

FAs, fatty acids; DMSO, dimethylsulfoxide control; $\mathrm{SCD} 1_{\text {inhib }}, \mathrm{SCD} 1$ inhibitor. 


\section{Lipid Class Composition}

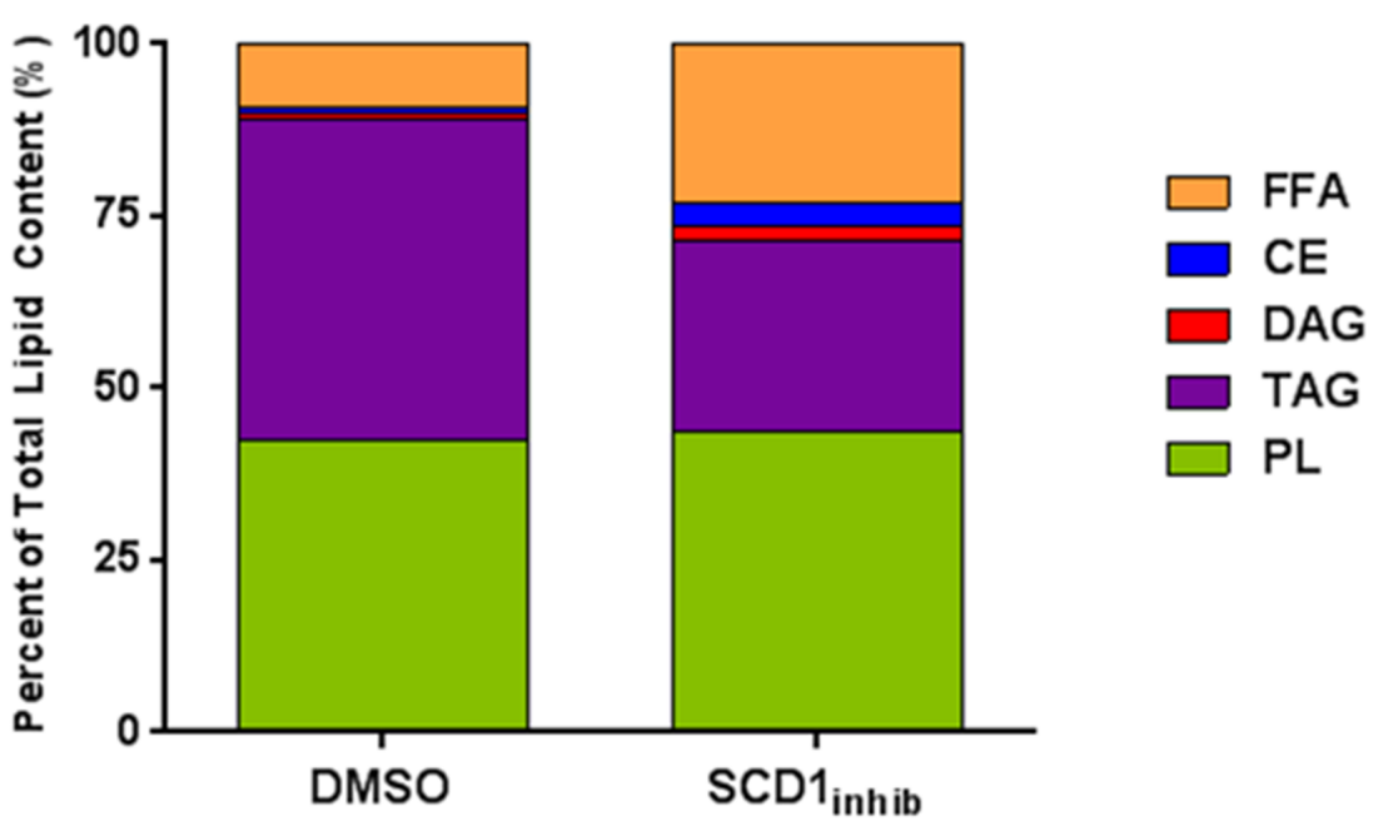

Figure 4.1. Relative composition of lipid classes. The relative composition of each lipid fraction expressed as a percentage of the total lipid content in differentiating adipocytes treated with or

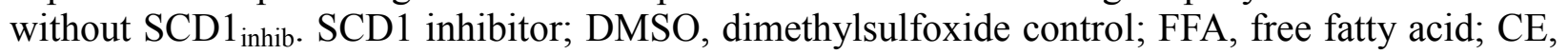
cholesteryl ester; DAG, diacylglycerol TAG, triacylglycerol; PL, phospholipid. 


\subsubsection{Effects of SCDI Inhibition on Saturated Fatty Acids (SFAs)}

SCD1 $1_{\text {inhib }}$ did not significantly alter total cellular SFA content at earlier time points; however, total cellular SFA levels were increased at day 7 compared to control cells ( 1.3-fold; $\mathrm{p}=0.02$ in post-hoc analysis). Examining each lipid fraction separately revealed significant treatment effects on SFAs in the PL, CE, and DAG fractions (Table 4.2). Additionally, SFA content within the DAG fraction was significantly increased as early as day 4. Although no treatment effects were noted for SFAs in the TAG and FFA fractions, post-hoc analyses revealed a significant decrease and increase, respectively, at day 7.

The effect of $\mathrm{SCD} 1_{\text {inhib }}$ on total cellular SFA content was predominantly driven by changes in the levels of two specific FAs: 16:0 and 18:0. Although there was no overall treatment effect on total cellular 16:0 levels, PL-16:0, TAG-16:0, or FFA-16:0, our post-hoc analysis revealed that $\mathrm{SCD} 1_{\text {inhib }}$ significantly decreased and increased TAG-16:0 and FFA-16:0 at day 7, respectively (Figure 4.2). There was a significant treatment effect on CE-16:0 and DAG-16:0 (Figures 4.2 D, F). Total cellular 18:0 levels in control adipocytes were significantly lower at later time points of differentiation ( 1.5 -fold decrease from day 0 to $7 ; \mathrm{p}=0.03$ ). However, $\mathrm{SCD} 1_{\text {inhib }}$ prevented this reduction at days 2 and 4 , and caused a $\sim 2.2$-fold increase in 18:0 at day 7 compared to control cells ( $<<0.01$; Figure $4.3 \mathrm{~A}$ ). The effects of $\mathrm{SCD} 1_{\text {inhib }}$ on total cellular 18:0 were mirrored in various fractions. PL-18:0 content was preserved during differentiation in SCD1-inhibited adipocytes, while TAG-18:0, CE-18:0 and DAG-18:0 levels were significantly increased (Figures $4.3 \mathrm{~B}, \mathrm{C}, \mathrm{D}$ and F). Although there was no overall treatment effect on FFA-18:0 (Figure 4.3 E), post-hoc analysis revealed a significant increase in levels at day 7 with $S C D 1_{\text {inhib. }}$ Other SFAs that were significantly altered in SCD1 inhibited adipocytes, albeit to a minor extent, included 14:0 and 15:0, which were decreased in both total cellular FA content as well as within the TAG fraction (data not shown). 
Table 4.2

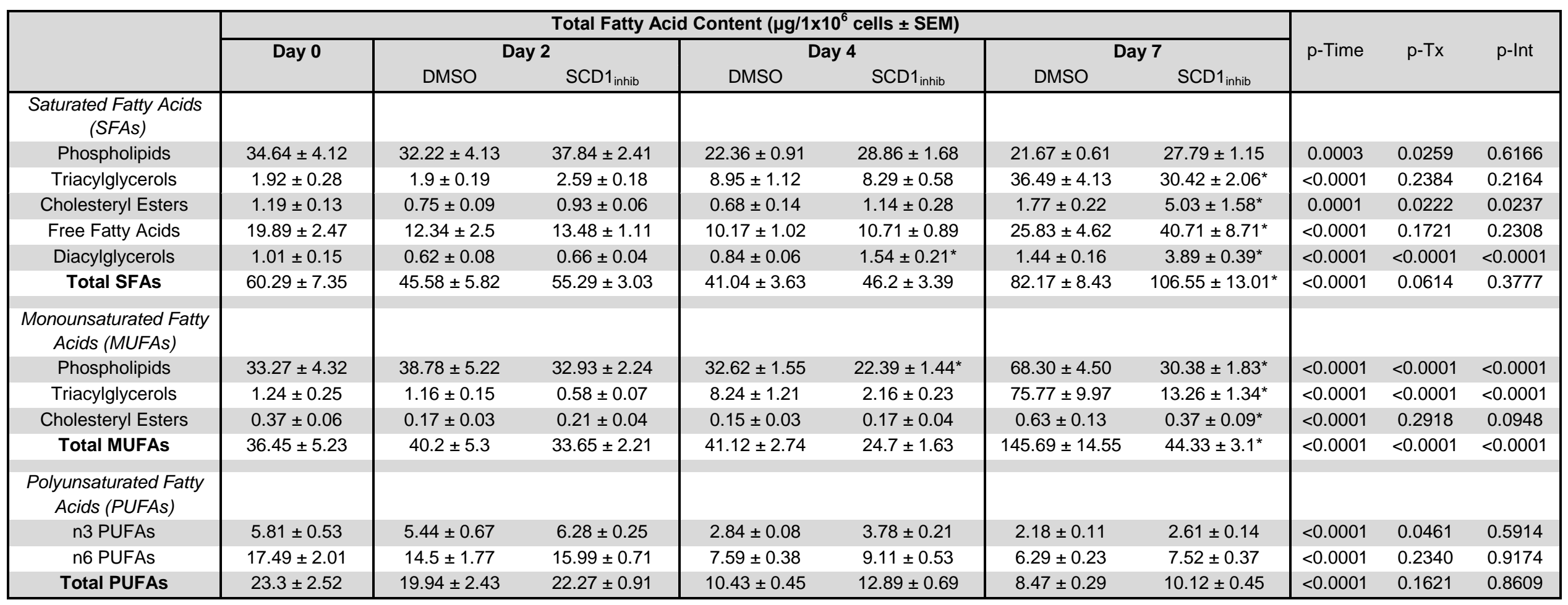

Total SFA, MUFA and PUFA Content of Lipid Fractions. Total content of SFAs, MUFAs and PUFAs in adipocytes treated

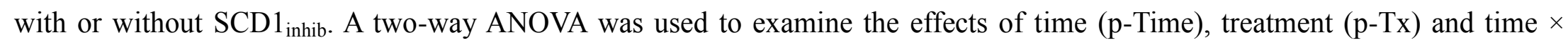

treatment interactions ( $\mathrm{p}$-Int). ${ }^{*}$ Indicates a significant difference by Fisher's Least Significant Difference post-hoc test $(\mathrm{p}<0.05)$.

SFAs, saturated fatty acids; MUFAs, monounsaturated fatty acids; PUFAs, polyunsaturated fatty acids; DMSO, dimethylsulfoxide

control; SCD1 $1_{\text {inhib }}$ SCD1 inhibitor. 


\section{A. Total Cellular 16:0 Content}

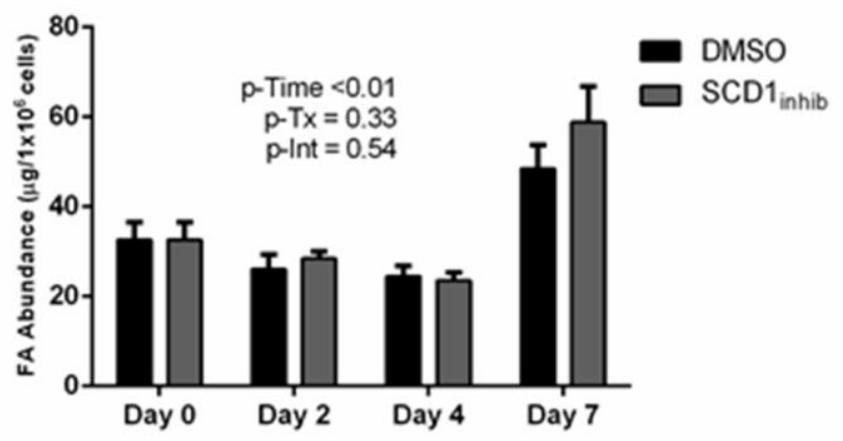

C. 16:0 Content in TAG

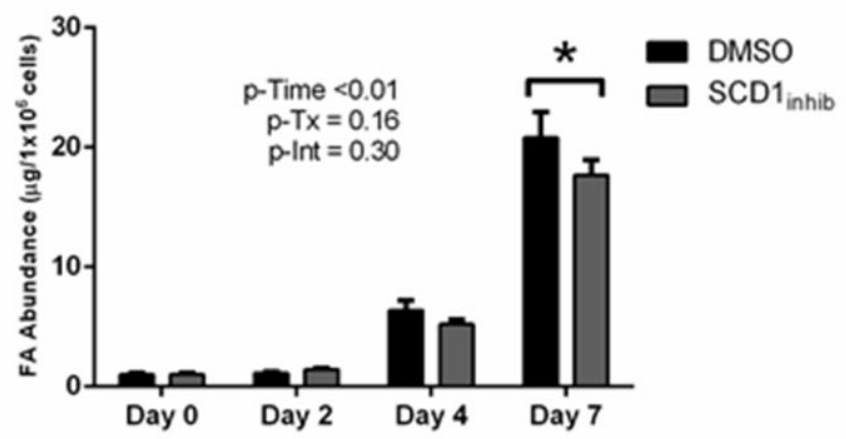

E. 16:0 Content in FFA

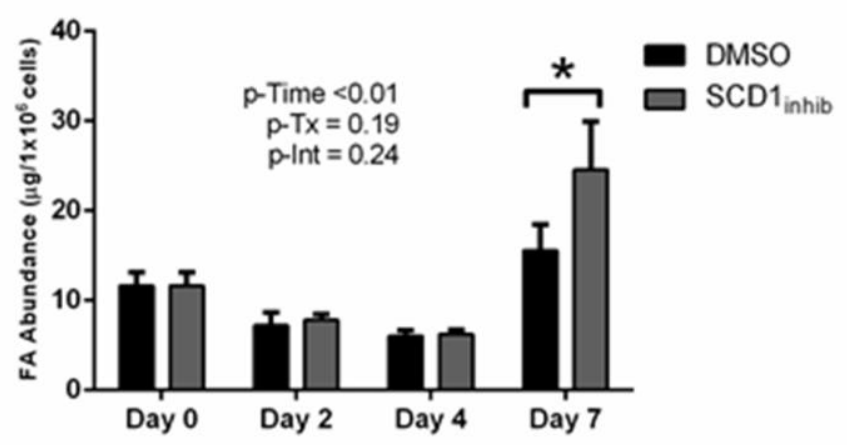

B. 16:0 Content in PL

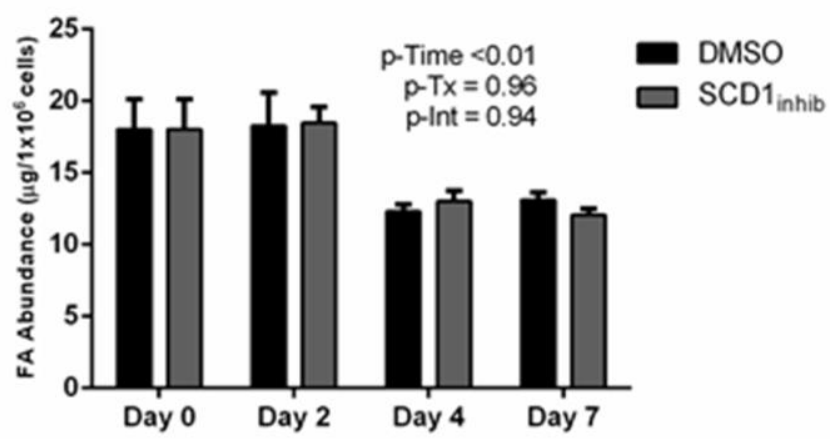

D. 16:0 Content in CE

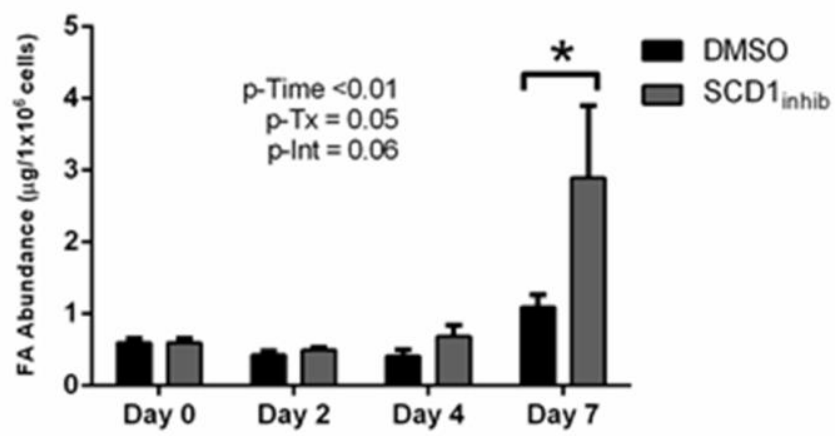

F. 16:0 Content in DAG

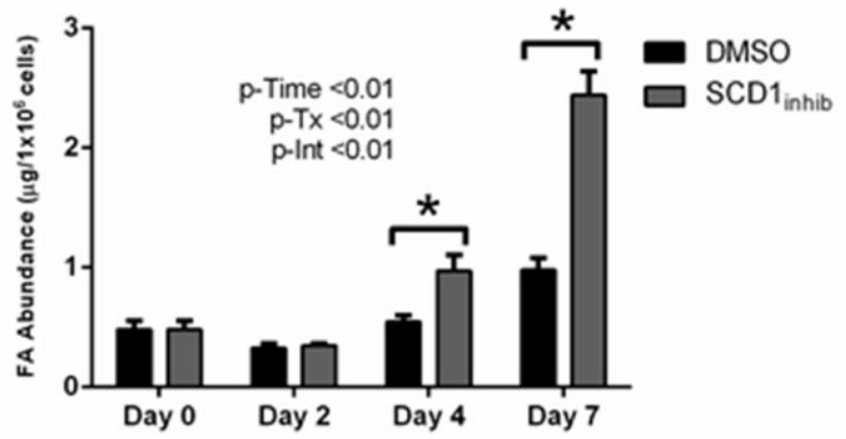

Figure 4.2: Total 16:0 abundance in adipocytes. Levels of palmitate (16:0) in (A) total cellular lipid content, (B) total PL content, (C) total TAG content, (D) total CE content, (E) total FFA content, and

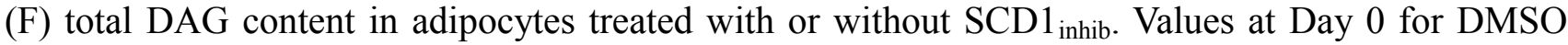
and $\mathrm{SCD} 1_{\text {inhib }}$ data are identical, but represented twice for ease of comparison. A two-way ANOVA was used to examine the effects of time (p-Time), treatment ( $\mathrm{p}-\mathrm{Tx})$ and time $\mathrm{x}$ treatment interactions (p-Int). *Indicates a significant difference by Fisher's Least Significant Difference post-hoc test $(\mathrm{p}<0.05)$. FA, fatty acid; SCD1 inhib, SCD1 inhibitor; DMSO, dimethylsulfoxide control; PL, phospholipid; TAG, triacylglycerol; CE, cholesteryl ester; FFA, free fatty acid; DAG, diacylglycerol. 
A. Total Cellular 18:0 Content

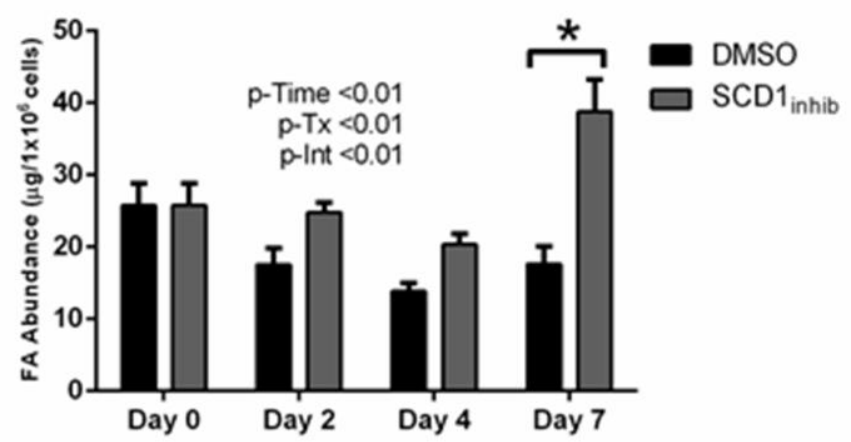

C. 18:0 Content in TAG

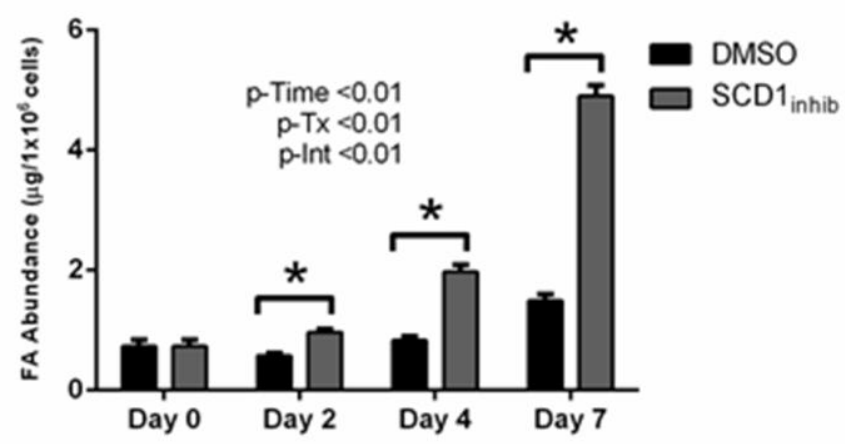

E. $\quad 18: 0$ Content in FFA

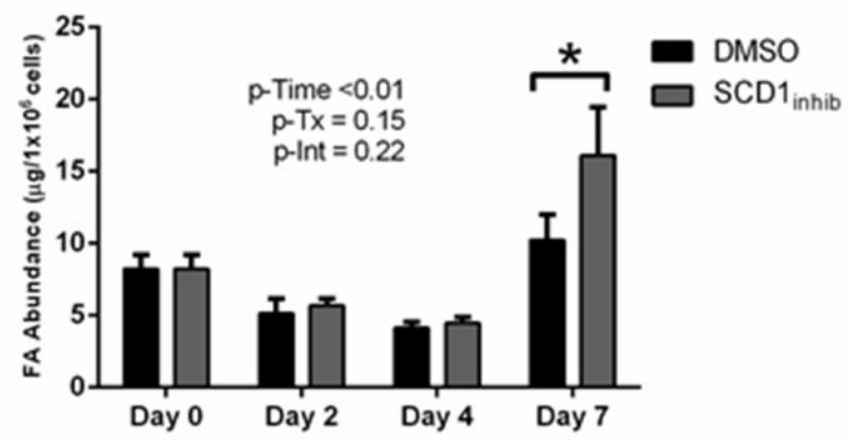

B. 18:0 Content in PL
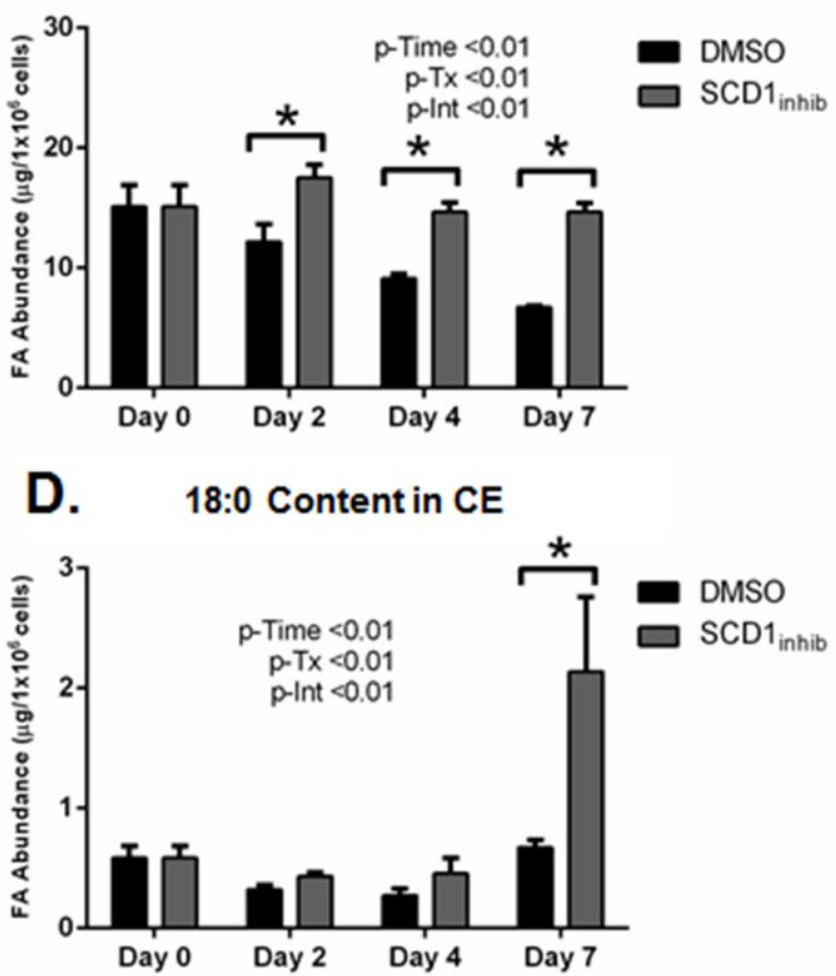

F. 18:0 Content in DAG

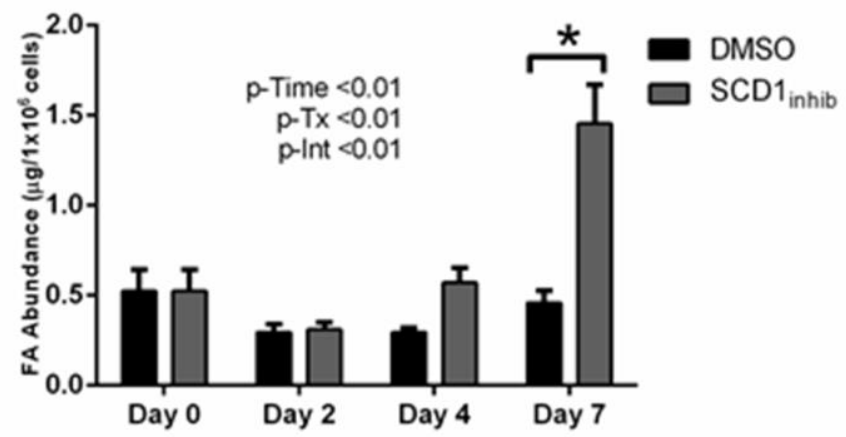

Figure 4.3: Total 18:0 abundance in adipocytes. Levels of stearate (18:0) in A) total cellular lipid content, B) total PL content, C) total TAG content, D) total CE content, E) total FFA content, and F) total DAG content in adipocytes treated with or without $\mathrm{SCD} 1_{\text {inhib. }}$. Values at Day 0 for DMSO and $\mathrm{SCD} 1_{\text {inhib }}$ data are identical, but represented twice for ease of comparison. A two-way ANOVA was used to examine the effects of time (p-Time), treatment ( $\mathrm{p}-\mathrm{Tx})$ and time $\mathrm{x}$ treatment interactions ( $\mathrm{p}$ Int). *Indicates a significant difference by Fisher's Least Significant Difference post-hoc test $(\mathrm{p}<0.05)$. FA, fatty acid; SCD1 inhib, SCD1 inhibitor; DMSO, dimethylsulfoxide control; PL, phospholipid; TAG, triacylglycerol; CE, cholesteryl ester; FFA, free fatty acid; DAG, diacylglycerol. 


\subsubsection{Effects of SCDI Inhibition on Monounsaturated Fatty Acids (MUFAs)}

While total cellular MUFA content increased throughout differentiation in the control condition ( 4.0 -fold increase from day 0 to $7 ; \mathrm{p}<0.001)$, this increase was prevented with SCD1 $1_{\text {inhib }}$ (Table 4.2). This was also observed for MUFA in the PL and TAG fractions (Table 4.2). Examining specific MUFAs revealed that $16: 1 \mathrm{n}-7$ was the primary contributor to the aforementioned changes in total MUFA content. In other words, while total cellular 16:1n-7 was increased during differentiation in control adipocytes ( 24.6 -fold from day 0 to 7 ; $p<0.001$ ), $\mathrm{SCD} 1_{\text {inhib }}$ prevented this increase (Figure 4.4 A). Again, these effects were also seen within various lipid fractions, where $\mathrm{SCD} 1_{\text {inhib }}$ blunted the accumulation of PL-16:1n-7 and TAG16:1n-7 during differentiation (Figures $4.4 \mathrm{~B}, \mathrm{C}$ ). There was no overall treatment effect on CE16:1n-7, although control cells did show a higher amount of CE-16:1n-7 at day 7 compared to $\mathrm{SCD} 1_{\text {inhib }}$ cells ( $\mathrm{p}=0.007$ in post-hoc analysis). No changes were seen with MUFAs in the DAG fraction. Other specific MUFAs altered by $\mathrm{SCD}_{\text {inhib }}$ included PL-17:1n-7 and PL-18:1n-9, which were both increased at various time points of differentiation in cells treated with

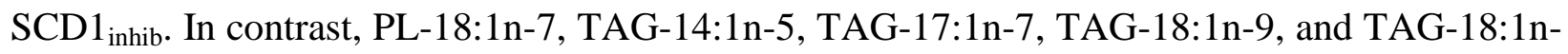
7 were generally lower in cells treated with $\mathrm{SCD} 1_{\text {inhib }}$ during differentiation (data not shown).

\subsubsection{Effects of SCD1 Inhibition on Polyunsaturated Fatty Acids (PUFAs)}

Total cellular PUFA content was significantly reduced over time in both control cells ( 2.8-fold decrease from day 0 to $7 ; \mathrm{p}<0.001)$ and in SCD1-inhibited adipocytes $(\sim 2.3$-fold decrease from day 0 to $7 ; \mathrm{p}<0.001$; Table 4.2). This was similarly reflected in total cellular $\mathrm{n}-6$ PUFA content. Interestingly, there was an overall treatment effect for n-3 PUFA content, where SCD1-inhibited adipocytes showed less of a reduction in n-3 PUFA (Table 4.2). SCD1 ${ }_{\text {inhib }}$ only caused significant changes in PUFA content within the PL fraction. Specifically, higher 22:5n-3 
content was observed in the PL fraction with SCD1 $1_{\text {inhib }}$ compared to control cells, while 18:3n-3 content was significantly lower at day 7. The levels of specific n-6 PUFAs were also impacted by $S C D 1_{\text {inhib. }}$ Specifically, the levels of $18: 2 n-6,18: 3 n-6,20: 3 n-6,20: 4 n-6$ and 22:4n-6 were significantly higher with $\mathrm{SCD} 1_{\text {inhib }}$ at day 7 compared to control cells (data not shown).

\section{A. Total Cellular 16:1n-7 Content}

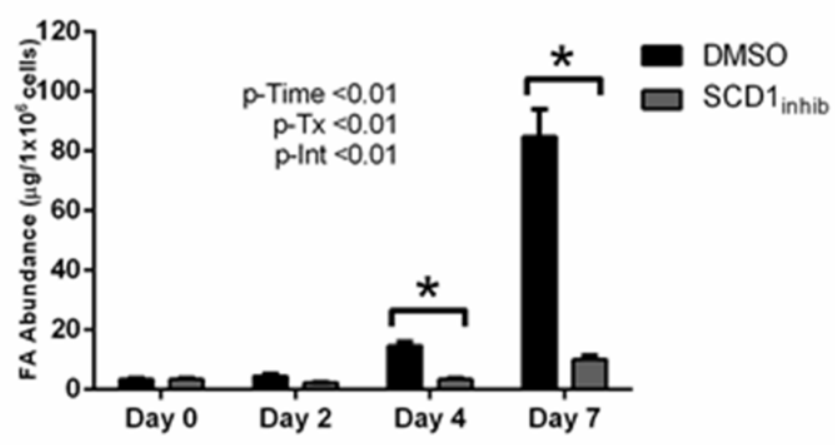

C. 16:1n-7 Content in TAG

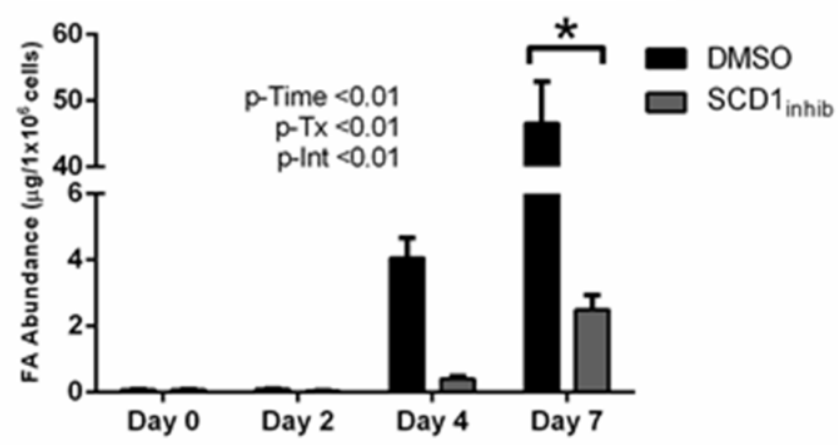

B. 16:1n-7 Content in PL

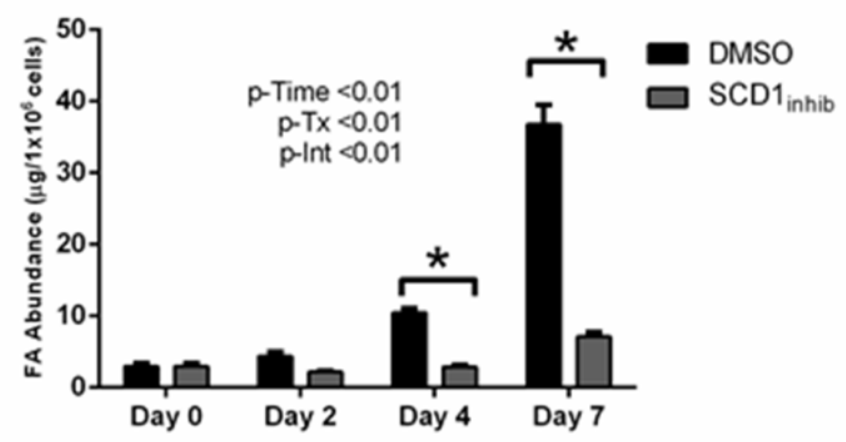

D. 16:1n-7 Content in CE

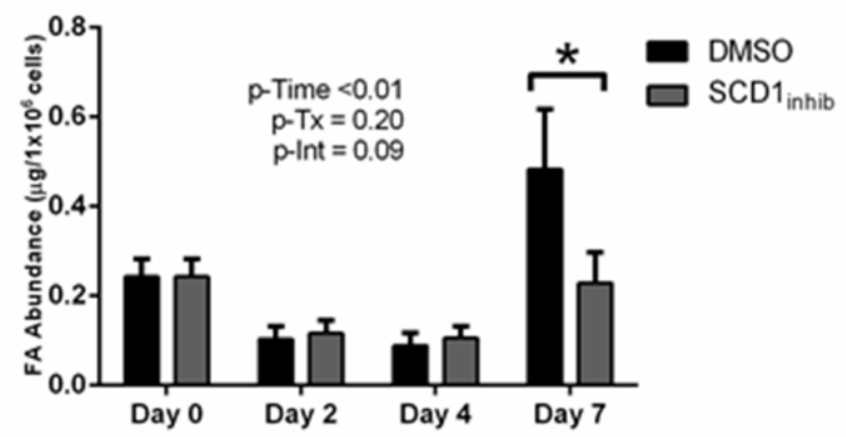

Figure 4.4: Total 16:1n-7 abundance in adipocytes. Levels of palmitoleate (16:1n-7) in (A) total cellular lipid content, (B) total PL content, (C) total TAG content, and (D) total CE content in

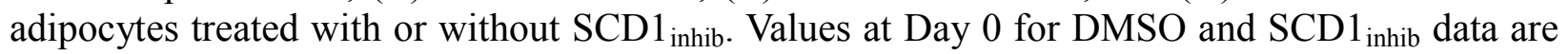
identical, but represented twice for ease of comparison. A two-way ANOVA was used to examine the effects of time (p-Time), treatment $(p-T x)$ and time $x$ treatment interactions (p-Int). *Indicates a significant difference by Fisher's Least Significant Difference post-hoc test $(\mathrm{p}<0.05)$. FA, fatty acid; SCD1 $1_{\text {inhib }}$, SCD1 inhibitor; DMSO, dimethylsulfoxide control; PL, phospholipid; TAG, triacylglycerol; CE, cholesteryl ester. 


\subsubsection{SCD1 Inhibition Down-Regulates Genes Involved in PL Biosynthesis}

Given the significant changes in absolute levels of cellular PL content observed with SCD1 $1_{\text {inhib }}$ treatment, we examined a previously generated microarray dataset in SCD1-inhibited adipocytes (NCBI Gene Expression Omnibus ID: GSE51905)). We focused our analysis on genes that were i) known to be involved in PL biosynthesis, and ii) that were differentially expressed with SCD1 inhibition. We found that SCD1 inhibition significantly down-regulated the expression of Agpat3, Agpat9, and $D g k \delta$ in differentiated adipocytes. We validated microarray data using RT-PCR and found that Agpat3, Agpat9 and Dgk $\delta$ expression were significantly reduced by 1.5-, 1.9-, and 1.4-fold, respectively $(\mathrm{p}<0.05)$.

\subsubsection{Effects of SCD1 Inhibition on Insulin Signalling and Cell Stress}

Increased levels of cellular DAGs have previously been associated with inhibition of insulin signalling [207, 208]. Due to the significant increases in cellular DAG content observed in SCD1-inhibited adipocytes, we examined whether SCD1 ${ }_{\text {inhib }}$ treatment influenced the activity of two key regulators of insulin signalling and cell stress: AKT and JNK. We observed a significant decrease in the phosphorylation of $\mathrm{p}-\mathrm{AKT}^{\mathrm{Ser} 473}(\mathrm{p}=0.02)$ in SCD1-inhibited adipocytes. No changes were observed for $\mathrm{p}-\mathrm{AKT}^{\mathrm{Thr} 308}$ or p-JNK (Figure 4.5).

\subsection{Discussion}

The present investigation adds to the existing body of knowledge regarding the role of SCD1 as a regulator of adipocyte lipid metabolism and cellular function. SCD1 inhibition had the greatest impact in the latter stages of adipogenesis, where a significant reduction in total 
A. p-AKT Thr308

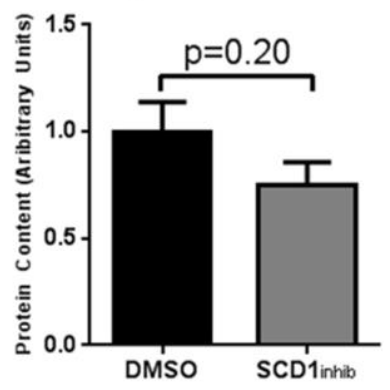

C.

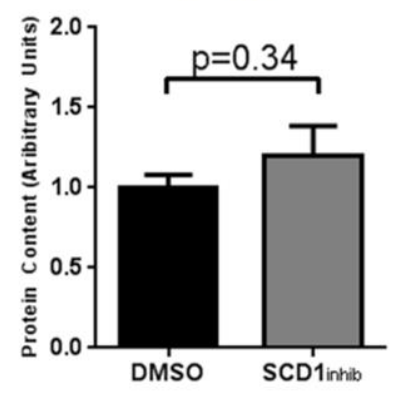

B. $\quad$-AKT Ser473
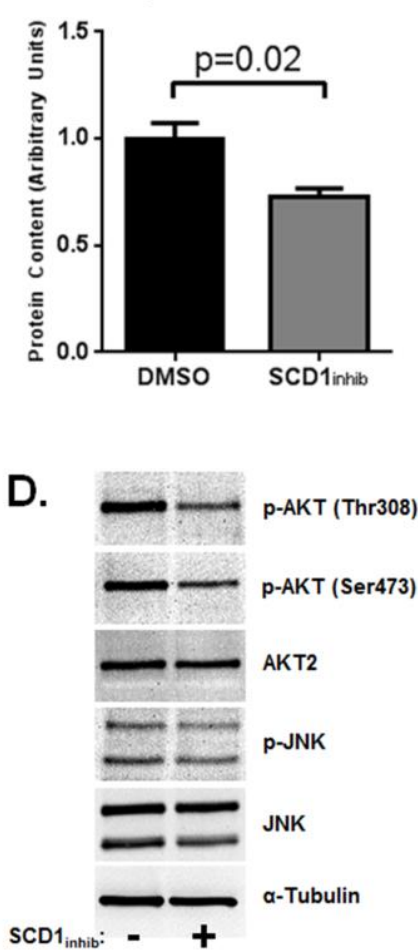

Figure 4.5: p-AKT and p-JNK Protein Content. Protein content of (A) p-AKT (Thr308), (B) pAKT (Ser473) and (C) p-JNK in adipocytes treated with or without SCD1 $1_{\text {inhib. Phosphorylated }}$ proteins are expressed relative to their total protein counterpart. Representative western blots are shown in (D). SCD1 $1_{\text {inhib}}$, SCD1 inhibitor; DMSO, dimethylsulfoxide control; AKT, protein kinase B; JNK, c-Jun N-terminal kinase.

cellular lipid content was observed at day 7. Moreover, we found that SCD1 inhibition decreased the levels of total cellular PL and TAG, while concomitantly increasing total cellular CEs, FFAs and DAGs. Although the increase in DAG did not alter a common marker of cell stress, insulin signalling may have been partially repressed. Moreover, SCD1 inhibition resulted in a significant remodelling of the FA composition of each lipid fraction. Altogether, these findings have important implications since it is known that changes in cellular FA composition can affect a myriad of biological pathways via the regulation of gene expression and membrane fluidity [64, 
125]. Results from this work reinforce that continued investigation into the underlying metabolic changes seen in adipocytes with altered SCD1 activity is warranted.

\subsubsection{SCD1 Inhibition Alters Total Lipid Content within Lipid Fractions}

We first examined total cellular lipid content as well as the levels of common lipid fractions (i.e., PL, TAG, CE, FFA, and DAG) in SCD1-inhibited adipocytes. The most significant changes were observed in the latter stages of differentiation (i.e., after day 4). This is not surprising given that this time point coincides with when differentiating adipocytes accumulate most of their lipid, as reflected by visible lipid droplets in the vast majority of cells $[7,209]$. We found that total cellular lipid content was increased substantially by day 7 of differentiation in control cells; however, SCD1 inhibition significantly blunted this accumulation. The effects of SCD1 inhibition were predominantly reflected in the PL, TAG, and DAG fractions. The decrease in total TAG content was not unexpected, as reduced SCD1 activity was previously shown to decrease hepatic TAG levels in rodents [10]. Furthermore, we previously reported that SCD1 inhibition in fully differentiated 3T3-L1 adipocytes downregulated the expression of various genes involved in TAG biosynthesis[201]. However, our previous work did not examine other lipid fractions or the FA content of these different fractions in SCD1-inhibited adipocytes. This is necessary in order to more thoroughly understand the consequences of SCD1 inhibition on adipocyte lipid profiles.

\subsubsection{Proposed Mechanism Contributing to Reduced PL and Increased Cellular DAG}

Although SCD1 inhibited adipocytes had lower total absolute PL levels at day 7 compared to control cells, we found that the relative proportion of the PL fraction was unaltered. 
This is intriguing and suggests that despite reductions in total PL, adipocytes maintain $\sim 40 \%$ of their total lipid as PLs. Nevertheless, the reduction in absolute PL observed in adipocytes treated with $\mathrm{SCD} 1_{\text {inhib }}$ prompted us to examine a previously generated microarray dataset in order to gain more insight into the potential transcriptional mechanisms underlying this change. We found that several genes involved in PL biosynthesis were significantly down-regulated with SCD1 inhibition, including acylglycerol-3-phosphate acyltransferase 3 (Agpat3) and Agpat9, which encodes a glycerol-3-phosphate acyltransferase (GPAT3). Both of these genes are involved in phosphatidic acid (PTA) production for PL biosynthesis [210]. A previous study by Ntambi et al. reported that hepatic Gpat was down-regulated in Scd1-/- mice [15]; however, to our knowledge this is one of the first demonstrations to show that reduced SCD1 activity also down-regulates the expression of adipocyte Gpat genes. Additionally, diacylglycerol kinase delta ( $D g k \delta)$, which produces PTA by phosphorylating DAG [211], was also down-regulated with SCD1 inhibition. We propose that, together, decreased expression of Agpat3, Agpat9, and Dgk $\delta$ reduces PL biosynthesis and thus provides a possible explanation for the decrease in absolute PL levels at day 7 (Figure 4.6). Additionally, the decrease in Agpat3 and Agpat9 may also contribute to the reduction in total cellular TAG, since the enzymes encoded by these genes are involved in TAG biosynthesis [212].

The increase in total DAG content seen with SCD1 inhibition may partially stem from a decrease in $D g k \delta$ expression, since a reduction in the conversion of DAG to PTA would cause an accumulation of DAG [211]. As such, DGK $\delta$ is thought to act as an indirect regulator of cell function by controlling levels of DAG and PTA [211]. This is important since increased cellular DAG has been shown to inhibit the insulin signalling pathway [207, 208]. Given that increased DAG may impair insulin signalling and/or cause cell stress, we examined whether SCD1 
inhibition altered JNK or AKT activity. While we observed no changes in p-JNK or p-AKT ${ }^{\text {Thr308, }}$ we detected a significant reduction in $\mathrm{p}-\mathrm{AKT}^{\mathrm{Ser} 473}$ in SCD1-inhibited adipocytes. This suggests that the increased cellular DAG content seen in SCD1-inhibited adipocytes may partially inhibit basal insulin signalling. This aligns with a previous report showing that human adipocytes lacking SCD1 had reduced insulin sensitivity [125]. In contrast, previous work by Hyun et al. has shown that the use of an SCD1-inhibitor (different from the one used in the present investigation) in 3T3-L1 cells can enhance basal glucose uptake [27]; however the mechanism involved did not appear to stem from changes in AKT phosphorylation [27].

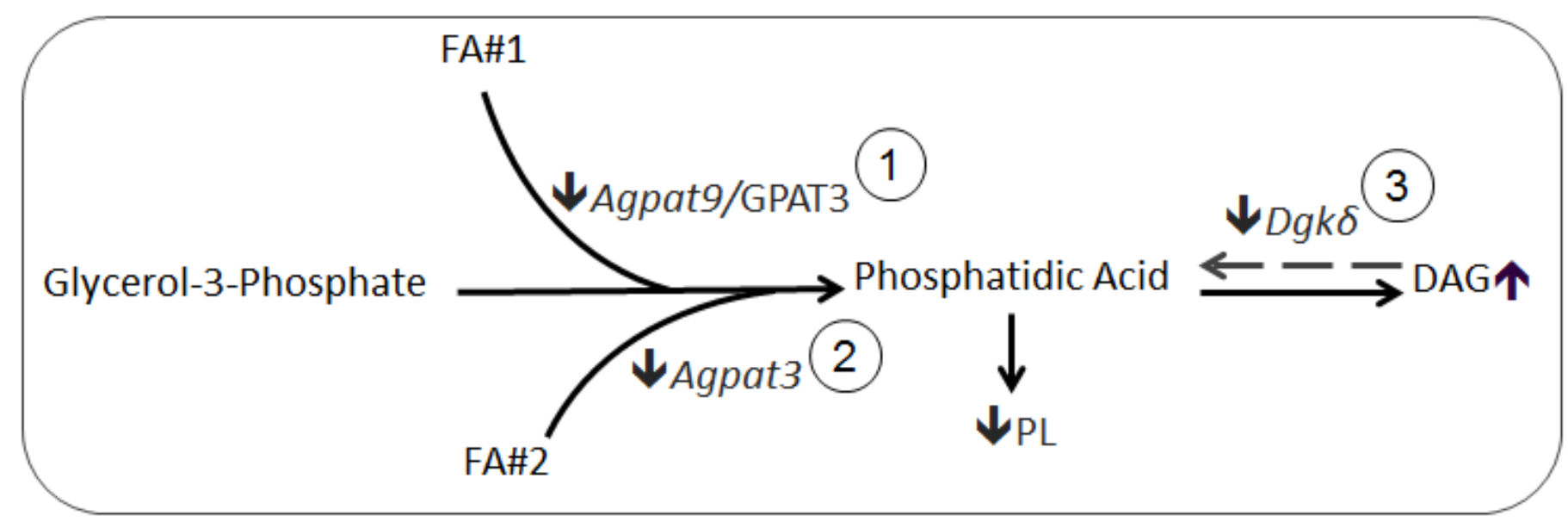

Figure 4.6. Proposed mechanism contributing to the reduction in PL and increase in DAG. Decreased expression of (1) Agpat9 and (2) Agpat3 would decrease the production of phosphatidic acid and total phospholipid (PL) content. Decreased expression of (3) $D g k \delta$ would result in reduced conversion of DAG to phosphatidic acid, and therefore an accumulation of DAG. FA, fatty acid; PL, phospholipid; DAG, diacylglycerol; Agpat, acylglycerol-3-phosphate acyltransferase; GPAT3, glycerol-3-phosphate acyltransferase 3; $D g k \delta$ diacylglycerol kinase delta. 


\subsubsection{SCD1 Inhibition Alters SFA, MUFA and PUFA Content in Various Lipid Fractions}

We also investigated whether SCD1 inhibition in differentiating adipocytes altered the content of SFA, MUFA, and PUFA within the various lipid fractions. Given that SCD1 converts SFA to MUFA, it was expected that altered SCD1 activity would modify the content of these FA classes. While SCD1 inhibition did significantly increase total SFA and SFA content within the CE, FFA and DAG fractions at day 7, we noted a decrease in TAG-SFA at day 7. The increases in total MUFA content, as well as TAG-MUFA, seen during adipogenesis were strongly blunted with SCD1 inhibition. This was expected since MUFA synthesized by SCD1 are thought to be the primary substrates for TAG biosynthesis [10, 101]. Additionally, although there was an overall treatment effect on PL-SFA, we did not observe any significant changes in PL-SFA content at specific time points of differentiation. We hypothesize that this may stem from decreases in certain SFAs (e.g., 14:0) that balance the increases observed with other SFAs (e.g., 18:0). An overall treatment effect was seen for total PL-MUFA, where SCD1 inhibition prevented the increase seen in control cells at day 7. This indicates that although the relative proportion of PL appears to be held steady in SCD1-inhibited adipocytes, the FA profiles within the PL fraction are remodelled.

\subsubsection{SCD1 Inhibition Alters 18:0 Levels in Various Lipid Fractions}

We were also interested in examining the extent to which SCD1 inhibition affected the composition of specific FAs within the various lipid fractions. While the levels of many FAs in differentiating adipocytes were only minimally changed with SCD1 inhibition, we found that 18:0 and 16:1n-7 levels were dramatically altered in several lipid fractions. Indeed, SCD1 inhibition increased total cellular 18:0 content in adipocytes, as well as 18:0 levels in PL, TAG, 
$\mathrm{CE}$ and DAG fractions, as early as day 2 of differentiation. While we previously reported that total cellular 18:0 content was increased in fully differentiated SCD1-inhibited adipocytes [201], the present work reveals these effects can be detected as early as day 2 of adipogenesis and is seen in several lipid fractions. Increased 18:0 within adipocytes appears to be one of the most significant modifications caused by SCD1 inhibition. Future investigations that intend to further characterize the effects of 18:0 in adipocytes are warranted in order to gain a better understanding of how altered 18:0 content during SCD1 inhibition may impact adipocyte function.

\subsubsection{SCD1 Inhibition Alters 16:1n-7 Levels in Adipocytes but not in Culture Media}

16:1n-7 is one of the most abundant FAs found within PLs and TAGs of differentiated 3T3-L1 adipocytes [202]. During differentiation, the control adipocytes showed a substantial increase in total cellular 16:1n-7 content. Further, this increase was reflected in the PL and TAG fractions of control adipocytes. However, SCD1 inhibition prevented these increases. These results align with findings that treating 3T3-L1 adipocytes with sterculic acid, a natural inhibitor of SCD1, caused a 90\% decrease in 16:1n-7 content [167]. It has been previously suggested that 16:1n-7 and its related metabolites (e.g., 1-acyl-2-16:1-sn-phosphatidylinositol) are lipid signalling molecules that may regulate whole-body glucose homeostasis [13, 164]. Indeed, Cao et al. proposed that 16:1n-7 is a "lipokine", i.e., a FA that is actively secreted from adipocytes and may have endocrine functions [13]. Given this hypothesis, we also examined the content of secreted 16:1n-7 in the cell culture media. Interestingly, we found that the amount of 16:1n-7 detected in adipocyte secretion media was not different between $S C D 1_{\text {inhib }}$ and control cells 
(unpublished observation). Therefore, despite dramatic reductions in cellular 16:1n-7 production, adipocytes appear to preserve the amount of secreted 16:1n-7.

Although PUFA content was reduced throughout differentiation in both control and SCD1-inhibited adipocytes, we did not observe an overall treatment effect of SCD $1_{\text {inhib }}$ on either total cellular PUFA content or n-6 PUFA content. In contrast, we did find a weak treatment effect for n-3 PUFA, which were reduced to a lesser degree in SCD1-inhibited adipocytes compared to control cells. While a reciprocal relationship between SCD1 and PUFA has been previously described in hepatocytes [213] as well as 3T3-L1 adipocytes [71], our results suggest that this may primarily concern n-3 PUFAs.

Limitations to the current study include the need to translate our cellular findings to an in vivo context. AT is not only comprised of adipocytes, but also includes macrophages, endothelial cells and mesenchymal stem cells, amongst others [140]. These different cell-types are in constant communication with one another (i.e., cellular crosstalk); thus perturbations in one celltype are bound to influence the activity of other cell-types. Studies investigating the effects of reduced SCD1 activity in adipocyte-macrophage crosstalk would be particularly valuable. Additionally, we note that the addition of internal FA standards prior to commencing TLC may partially underestimate FA concentrations; however, any effect would be consistent throughout the study and should not impact our ability to detect differences among treatments or time points. Finally, since we observed changes in total PL content and composition, it will be interesting to examine the effects of SCD1 inhibition on the levels of specific PL species (phosphatidylinositol, phosphatidylcholine, phosphatidylethanolamine, etc.), as different PLs have distinct roles within a cell. 


\subsubsection{Summary and Perspectives}

In conclusion, we report that SCD1 inhibition in differentiating adipocytes leads to significant changes in the content of various lipid fractions, as well as significant FA remodelling within these fractions. However, the full extent of how these changes in lipid profiles impact adipocyte function remains unclear. For example, a reduction in total TAG content could be considered advantageous in combating obesity, but if adipocytes are unable to store TAG effectively then this could lead to ectopic fat deposition and promote metabolic complications [125]. While SCD1-inhibited adipocytes maintain the same relative proportion of PLs as control adipocytes, the FA composition of the PL fraction was altered and the absolute content of PLs was reduced, possibly via down-regulated PL biosynthesis. Finally, while increased cellular DAG content was observed in SCD1-inhibited adipocytes, this increase did not appear to activate JNK or completely impair basal insulin signalling. Collectively, these results add to the growing

body of knowledge regarding SCD1 function in adipocytes and highlight its important role in regulating global adipocyte lipid composition. 


\section{CHAPTER 5:}

SCD1 MEDIATES THE INFLUENCE OF EXOGENOUS SATURATED AND MONOUNSATURATED FATTY ACIDS IN ADIPOCYTES: EFFECTS ON CELLULAR STRESS, INFLAMMATORY MARKERS, AND FATTY ACID ELONGATION

As submitted to the Journal of Nutritional Biochemistry:

Jessica C. Ralston, Adam H. Metherel, Ken D. Stark and David M. Mutch. SCD1 mediates the influence of exogenous saturated and monounsaturated fatty acids in adipocytes: Effects on cellular stress, inflammatory markers, and fatty acid elongation 


\subsection{Abstract}

Palmitate (PA), stearate (SA), palmitoleate (PMA) and oleate (OA) are among the most abundant fatty acids (FAs) in adipose tissue (AT). These FAs differentially regulate AT inflammation by altering adipocyte signalling pathways and the secretion of pro-inflammatory cytokines. Intracellular levels of these FAs are controlled, in part, by stearoyl-CoA desaturase 1 (SCD1). Therefore, SCD1 may have an important role mediating FA-regulation of adipocyte inflammation. Our goal was to investigate if the influence of PA, SA, PMA and OA on FA metabolism, inflammation and cellular stress was altered with reduced SCD1 activity in 3T3-L1 adipocytes. Real-time RT-PCR, immunoassays, gas chromatography and western blotting were used to examine the expression and secretion of common inflammatory markers, as well as FA profiles and markers of cellular stress. FA treatments differentially affected inflammatory markers and FA profiles in SCD1-inhibited adipocytes. Specifically, SA significantly increased the expression of $C c l 5$ (5.3-fold) and Mcp-1 (3.2-fold), and the secretion of IL-6 (17.8-fold) and MCP-1 (4.0-fold) in SCD1-inhibited adipocytes compared to controls. The pro-inflammatory effects observed with SA are particularly notable given that SCD1-inhibited adipocytes increase elongation of PA to SA, as determined using $\mathrm{U}_{-}{ }^{13} \mathrm{C}-\mathrm{PA}$. The effects of PA, PMA and OA were not as substantial as those of SA, although PA did significantly increase $C c l 5$ (2.7-fold) and Mcp-1 (1.2-fold) expression in SCD1-inhibited adipocytes. None of the FAs influenced markers of cellular stress. Collectively, these results emphasize the differential effects of individual FAs, and highlight how SCD1 influences their regulation of adipocyte inflammation. 


\subsection{Introduction}

Obesity is considered a state of chronic low-grade inflammation and is a major worldwide health concern [3, 4]. Adipose tissue (AT) hypertrophy is associated with the dysregulation of cytokine and chemokine production and secretion [3, 198]. These secreted proteins play a key role in the paracrine dialogue between the various cell types comprising AT (e.g., adipocytes, macrophages, endothelial cells, T-cells, etc.), which ultimately regulates numerous biological processes including insulin signalling and cellular stress [3]. As such, the regulation of inflammatory signalling pathways is critically important for overall AT metabolism. Fatty acids (FAs) are one class of compounds that are documented to regulate adipocyte inflammation [64, 214, 215]. For example, previous work by van Dijk et al. showed that consuming a diet high in SFAs increased pro-inflammatory gene expression in human AT, yet a diet high in MUFAs had an anti-inflammatory effect [214]. Additionally, we previously showed that the two most common SFAs, palmitate (PA) and stearate (SA), differentially regulated inflammatory signalling pathways in 3T3-L1 preadipocytes [64]. Together, the existing evidence demonstrates that individual FAs have distinct effects on AT inflammation and metabolism. This emphasizes the need to examine individual FAs in order to delineate their links with inflammation and adipocyte metabolism.

FAs that are derived from the diet or from de novo lipogenesis are primarily stored in adipocytes as triacylglycerols (TAGs) [198]. As such, adipocytes express a panel of genes that enable the production, metabolism and storage of FAs [7]. Stearoyl-CoA desaturase 1 (SCD1) plays a central role in FA metabolism by converting the SFAs PA and SA, to the MUFAs palmitoleate (PMA) and oleate (OA), respectively [28]. SCD1 has been previously associated 
with alterations in cellular inflammation and stress, where primary adipocytes isolated from whole-body SCD1-deficient mice were shown to have reduced inflammation compared to wildtype adipocytes [120,193]. Additionally, Malodobra-Mazur et al. have recently shown that SCD1 can mediate inflammation in 3T3-L1 adipocytes by regulating DNA methylation [162]. Given the purported link between SCD1 and inflammation, as well as SCD1's central role in FA metabolism, it is of interest to determine if SCD1 mediates the relationships between individual FAs and inflammation.

FA substrates and products of SCD1 are among the most abundant FAs in AT [64, 118, 125, 202, 216]. Consequently, FA regulation of adipocyte signalling pathways may be altered when SCD1 activity is compromised. Therefore, our objective was to examine if SCD1 inhibition in adipocytes altered the influence of PA, PMA, SA and OA on lipid metabolism, cellular stress and inflammation. We hypothesized that adipocytes with compromised SCD1 activity would show greater inflammatory and stress responses to FAs (in particular SFAs) compared to control adipocytes. Our results not only highlight the complex associations between SCD1, FAs, inflammation, and adipocyte metabolism, but demonstrate how SCD1 has an important role regulating the effects of SFAs and MUFAs in adipocytes.

\subsection{Materials and Methods}

\subsubsection{Chemicals and Cell Culture Reagents}

A specific SCD1 inhibitor (CAY10566) was purchased from Cayman Chemical (Ann Arbor, MI, USA), as previously described [201]. The murine 3T3-L1 pre-adipocytes were obtained from ATCC (Rockville, MD, USA). Cell culture reagents including Dulbecco's modified Eagle's medium (DMEM), penicillin-streptomycin (pen-strep), and 0.25\% trypsin- 
ethylenediaminetetraacetic acid solution were purchased from Hyclone laboratories (Logan, UT, USA). Other cell culture reagents including 3-isobutyl-1-methylxanthine (IMBX), dexamethasone (Dex), human insulin, fatty acid-free bovine serum albumin (BSA; $\geq 98 \%$ purity), fetal bovine serum (FBS), dimethylsulfoxide (DMSO; $\geq 99.9 \%$ purity), and all FAs (PA, PMA, SA, OA, and U- ${ }^{13}$ C-labeled PA) were purchased from Sigma Aldrich (St. Louis, MO, USA). The following primary antibodies were bought from Cell Signaling Technology (Danvers, MA, USA): p-JNK (\#4671), JNK (\#9252), p-p38 MAPK (\#9211), p38 MAPK (\#9212), p-STAT3 (\#9138), STAT3 (\#8768), p-ERK1/2 (\#9101), ERK1/2 (\#4695) and SCD1 (\#2438). Primary antibodies for $\alpha$-tubulin (\#ab7291) and ELOVL6 (\#ab69857) were purchased from Abcam (Toronto, ON, Canada).

\subsubsection{Cell Culture Experiments}

The SCD1 inhibitor (SCD1 ${ }_{\text {inhib }}$ ) was diluted to a stock concentration of $10 \mu \mathrm{M}$ in DMSO. A final working concentration of $10 \mathrm{nM} \mathrm{SCD} 1_{\text {inhib }}$ was created by further diluting the stock solution in adipocyte culture media, as previously described [201]. 3T3-L1 adipocytes were cultured in $5 \% \mathrm{CO}_{2}$ and $100 \%$ humidity at $37^{\circ} \mathrm{C}$ throughout all experiments. Prior to treatments, cells were maintained in basic media consisting of DMEM supplemented with 5\% heatinactivated FBS and $1 \%$ pen-strep. Adipocyte differentiation was conducted as previously described [201]. Briefly, differentiation was induced 2 days post confluence (i.e., day 0) using an established differentiation cocktail that consisted of IBMX $(0.5 \mathrm{mM})$, Dex $(1 \mu \mathrm{M})$, and human insulin $(5 \mu \mathrm{g} / \mathrm{ml})$ in basic media. Two days later (i.e., day 2), media was replaced with maintenance media which consisted of basic media supplemented with human insulin at $5 \mu \mathrm{g} / \mathrm{ml}$. On day 4, FBS was removed from the media and the remaining duration of the experiments were 
conducted in serum-free conditions. During each media change, cells were also treated with either $10 \mathrm{nM} \mathrm{SCD} 1_{\text {inhib }}$ or an equivalent volume of DMSO which served as the control condition.

Stock FA solutions of PA, PMA, SA and OA were made by solubilizing FAs in $100 \%$ ethanol (EtOH) [64]. 2\% BSA was prepared directly in serum-free media. FA stock solutions were diluted in the $2 \%$ BSA serum-free media to yield a FA:BSA molar ratio of $1: 3$, as previously described $[217,218]$. Adipocytes were treated with a final concentration of $250 \mu \mathrm{M}$ FAs for $48 \mathrm{~h}$, starting on day 5 . This dose was selected based on previous work in this adipocyte model as well as work from our laboratory [64, 192]. An equivalent volume of $100 \%$ EtOH diluted in $2 \%$ BSA serum-free media was used as the control. Cells were still maintained in either $10 \mathrm{nM} \mathrm{SCD} 1_{\text {inhib }}$ or an equivalent volume of DMSO for the duration of FA treatments. FA treatments did not cause cell toxicity, as confirmed using the Promega Cytotoxicity Assay (Madison, WI, USA). All experiments were performed with technical replicates in at least three different passages to ensure that results were not due to passage number.

\subsubsection{Lipid Extraction and Quantification}

Lipid extractions were conducted on day 7 using previously described protocols $[173$, 174, 201]. Samples were analyzed using an Agilent Technologies 7890B gas chromatography (GC) system (Agilent Technologies, Mississauga, ON, Canada) and quantified using methods previously outlined [201]. FA data was normalized by cell count for each treatment condition. FA data is reported as $\mu \mathrm{g} \mathrm{FA} / 1 \times 10^{6}$ cells $\pm \mathrm{SEM}$. 


\subsection{4 ${ }^{13}$ PA Elongation Experiments}

To assess if PA elongation to SA is up-regulated during SCD1 inhibition, control cells $\left(\mathrm{D}+{ }^{13} \mathrm{PA}\right)$ and SCD1-inhibited cells $\left(\mathrm{I}+{ }^{13} \mathrm{PA}\right)$ were treated with $250 \mu \mathrm{M}$ of a $\mathrm{U}-{ }^{13} \mathrm{C}$-labeled PA $\left({ }^{13} \mathrm{PA}\right)$ stable isotope tracer (Sigma Aldrich, St. Louis, MO, USA). Lipids were extracted from adipocytes using a modified Folch method [219] by the addition of $3 \mathrm{~mL}$ of chloroformmethanol with $10 \mathrm{mg}$ of docosatrienoic acid ethyl ester (Nu Chek Prep, Elysian, MN, USA) as an internal standard and $50 \mathrm{mg} / \mathrm{mL}$ butylated hydroxytoluene (Sigma-Aldrich, St. Louis, MO, USA) as an antioxidant. Following a $1 \mathrm{~min}$. vortex, $0.5 \mathrm{~mL}$ of $0.3 \mathrm{M}$ sodium phosphate buffer was added, inverted twice, and centrifuged for $5 \mathrm{~min}$. at 3,000 rpm. The lower organic, lipidcontaining phase was pipetted off and transferred to a new glass test tube for transesterification to FA methyl esters as described previously [220].

FA methyl esters were analyzed on a Varian 3900 GC (Bruker, Billerica, MA, USA) for the purpose of quantifying total SA, including the total concentration of co-eluted ${ }^{13} \mathrm{C}$-labeled SA and non-labeled SA. The GC settings were as previously described [221]. Peaks were identified by retention times through comparison to an external mixed standard sample (GLC-462, Nu Chek Prep Inc., Elysian, MN, USA).

Relative contributions of ${ }^{13} \mathrm{C}$-labeled SA to non-labeled SA were also analyzed, and based on concentrations determined from GC analysis, the concentration of the ${ }^{13} \mathrm{C}$-labeled SA $(M+16)$ was determined. A Varian 3800 gas chromatograph coupled to a Varian 4000 mass spectrometer with a quadrupole ion trap was utilized (Bruker, Billerica, MA, USA) [222]. The instrument was set for positive chemical ionization with isobutane as the ionizing gas and the reagent ion $\mathrm{C}_{4} \mathrm{H}_{9}{ }^{+}$(m/z 57) was mass selected to ionize the FA methyl esters [223]. The GC and mass spectrometer settings were as previously described [222]. 


\subsubsection{Immunoassays}

Media was extracted from adipocytes at day 7 and centrifuged at 1,200 rpm for $5 \mathrm{~min}$ to pellet any cell debris, and stored at $-80^{\circ} \mathrm{C}$ until analyzed. Samples were assessed for the presence of MCP-1, IL-6 and CCL5 using analyte-specific ProcartaPlex immunoassay kits that were purchased from eBioscience (San Diego, CA, USA) and analyzed on a multiplex system with xMAP technology (BioRad Laboratories, Mississauga, ON, Canada).

\subsubsection{RNA and Protein Extraction}

Total RNA and protein were extracted from adipocytes at day 7 using previously described protocols [218]. The Qiagen RNeasy Mini Kit (Qiagen, Mississauga, ON, Canada) was used to extract RNA. A Nanodrop and a detergent-compatible protein assay (BioRad Laboratories, Mississauga, ON, Canada) were used to quantify RNA and protein, respectively. Samples were stored at $-80^{\circ} \mathrm{C}$ prior to all analyses.

\subsubsection{Real-time RT-PCR}

Single stranded cDNA was synthesized from $1 \mu \mathrm{g}$ total RNA using a High-Capacity cDNA Reverse Transcription Kit (Life Technologies, Burlington, ON, Canada). Real-time RTPCR was conducted as described previously using a BioRad CFX96 RT-PCR detection system

and SSo FAST EvaGreen Supermix (BioRad Laboratories, Mississauga, ON, Canada) [201]. Primers for Scd1, Elovl6, Mcp-1, Il-6 and Ccl5 were designed using the online Roche Universal Probe Library and Assay Design Center and RplpO was used as a housekeeping gene. 


\subsubsection{Western Blot Analyses}

Western blot analyses were conducted as previously described [201]. Specific primary antibody dilutions were as follows: p-JNK - 1:500; JNK, p-p38 MAPK, p38 MAPK, p-STAT3, STAT3, p-ERK1/2, ERK1/2, SCD1 - 1:1000, $\alpha$-tubulin - 1:5000, and ELOVL6 - 1:8000. Relative band intensities were quantified using Alpha Innotech Software (San Leandro, CA, USA), and $\alpha$-tubulin was used as the internal control.

\subsubsection{Statistical Analyses}

FA and cytokine data was assessed using a one-way ANOVA and Fisher's Least Significant Difference post-hoc analysis. Significant differences in gene expression and protein content between treatment and control conditions were assessed using two-tailed student's ttests. A p-value $<0.05$ was considered statistically significant. All data are presented as mean \pm SEM.

\subsection{Results}

\subsubsection{SCD1 mediates the effects of SFAs and MUFAs on adipocyte FA metabolism}

We first examined how total lipid content is influenced by FAs in adipocytes treated with or without $\mathrm{SCD} 1_{\text {inhib. }}$ Adipocytes treated with only the inhibitor (i.e., I+EtOH) showed a small, but insignificant, reduction in total lipid content compared to the control condition (i.e., D+EtOH) (Table 5.1). Treating control adipocytes with FAs increased total lipid content, and similar increases were also seen in SCD1-inhibited adipocytes treated with FAs (i.e., I+PA, I+PMA, I+SA, or I+OA; Table 5.1). Interestingly, adipocytes treated with I+SA had a 
significantly higher total lipid content compared to adipocytes treated with I+PA, I+PMA, or $\mathrm{I}+\mathrm{OA}$.

As expected, treating control adipocytes with individual FAs led to a corresponding increase in their cellular abundance. Specifically, the D+PA, D+PMA, D+SA, and D+OA treatments caused significant increases in PA by 2.4-fold, PMA by 2.1-fold, SA by 3.6-fold, and OA by 5.3-fold, respectively (Table 5.1). Similarly, FA treatments in SCD1-inhibited adipocytes also caused substantial increases in PA by 3.1-fold, PMA by 7.2-fold, SA by 6.1-fold, and OA by 5.8-fold, respectively (Table 5.1).

Treating control adipocytes with SFAs (i.e., D+PA or D+SA) increased the abundance of their corresponding desaturated MUFAs, i.e., 2.0-fold increase in PMA $(\mathrm{p}<0.001)$ and $~ 4.6$-fold increase in OA ( $\mathrm{p}<0.001)$. Treating SCD1-inhibited adipocytes with SFAs (i.e., I+PA and I+SA) also increased the content of their corresponding desaturated MUFAs, albeit to a lesser extent (Table 5.1). The residual conversion of SFAs to MUFAs in SCD1-inhibited adipocytes treated with PA or SA did not stem from changes in $S c d 1$ gene expression or SCD1 protein content (data not shown). Rather, this conversion most likely stemmed from residual SCD1 activity when using this dose of inhibitor [201]. Furthermore, $S c d 2$ gene expression was also unaltered by SFA treatments during SCD1 inhibition (data not shown).

Interestingly, treating SCD1-inhibited adipocytes with PA caused a notable and statistically significant increase in cellular SA content compared to the I+EtOH condition (1.5fold increase, $\mathrm{p}=0.04$; Table 5.1). We previously reported that the estimated activity of ELOVL6 (i.e., estimated using the product-to-substrate ratio of FAs) was increased in SCD1-inhibited adipocytes [201]; therefore we repeated this experiment using $\mathrm{U}-{ }^{13} \mathrm{C}$-labelled PA. We observed a 5.7-fold increase in SA levels containing the stable isotope in $\mathrm{I}^{13} \mathrm{PA}$ compared to the $\mathrm{I}+\mathrm{EtOH}$ 


\section{Table 5.1}

\begin{tabular}{|c|c|c|c|c|c|c|}
\hline \multirow[b]{2}{*}{$\begin{array}{c}\text { Adipocyte Treatment } \\
\text { Condition }\end{array}$} & \multicolumn{6}{|c|}{ FA (measured by gas chromatography) } \\
\hline & PA & PMA & SA & OA & Other FAs & Total Lipids \\
\hline $\mathrm{D}+\mathrm{EtOH}$ & $22.11 \pm 1.21$ & $41.76 \pm 2.46$ & $3.94 \pm 0.20$ & $13.72 \pm 0.57$ & $27.81 \pm 1.48$ & $109.33 \pm 5.59$ \\
\hline $\mathrm{I}+\mathrm{EtOH}$ & $29.78 \pm 1.39$ & $15.27 \pm 1.15$ & $10.73 \pm 0.21$ & $20.46 \pm 0.71$ & $25.50 \pm 0.95$ & $101.74 \pm 4.09$ \\
\hline$p$-value & $<0.0001$ & $<0.0001$ & 0.0008 & 0.0165 & 0.1000 & 0.2409 \\
\hline $\mathrm{D}+\mathrm{PA}$ & $52.95 \pm 1.78$ * & $81.6 \pm 1.83^{*}$ & $5.63 \pm 0.13$ & $19.74 \pm 0.48$ & $35.59 \pm 0.97^{*}$ & $195.52 \pm 4.55$ * \\
\hline I+PA & $91.72 \pm 2.67 \ddagger$ & $26.34 \pm 1.75 \ddagger$ & $15.60 \pm 0.51 \ddagger$ & $20.76 \pm 1.03$ & $29.11 \pm 0.72 \ddagger$ & $183.52 \pm 5.78 \ddagger$ \\
\hline p-value & $<0.0001$ & $<0.0001$ & 0.0005 & 0.7957 & 0.0014 & 0.1906 \\
\hline D+PMA & $25.48 \pm 1.1$ & $88.6 \pm 2.81^{*}$ & $4.55 \pm 0.18$ & $12.4 \pm 0.5$ & $29.98 \pm 1.07$ & $161.01 \pm 5.56$ * \\
\hline I+PMA & $31.73 \pm 1.36$ & $109.96 \pm 4.05 \ddagger$ & $11.67 \pm 0.37$ & $13.89 \pm 0.67$ & $36.03 \pm 1.05 \ddagger$ & $203.30 \pm 6.78$ \\
\hline$p$-value & 0.0164 & $<0.0001$ & 0.0117 & 0.7026 & 0.0027 & $<0.0001$ \\
\hline $\mathrm{D}+\mathrm{SA}$ & $31.65 \pm 1.33^{*}$ & $47.27 \pm 3.72$ & $14.05 \pm 1.79$ * & $63.36 \pm 5.04$ * & $34.82 \pm 1.6^{*}$ & $191.15 \pm 7.38$ * \\
\hline $\mathrm{I}+\mathrm{SA}$ & $55.06 \pm 2.42 \ddagger$ & $16.42 \pm 2.01$ & $65.34 \pm 6.51 \mathrm{t}$ & $94.65 \pm 1.52 \ddagger$ & $36.88 \pm 1.32 \ddagger$ & $268.35 \pm 7.08$ \\
\hline$p$-value & $<0.0001$ & $<0.0001$ & $<0.0001$ & $<0.0001$ & 0.3116 & $<0.0001$ \\
\hline $\mathrm{D}+\mathrm{OA}$ & $26.79 \pm 1.25$ * & $37.14 \pm 2.16$ & $3.98 \pm 0.29$ & $73.32 \pm 6.04$ * & $27.85 \pm 1.41$ & $169.07 \pm 7.44$ * \\
\hline $\mathrm{I}+\mathrm{OA}$ & $34.62 \pm 1.66 t$ & $10.45 \pm 1.17$ & $10.28 \pm 0.17$ & $118.42 \pm 5.44 \ddagger$ & $24.75 \pm 0.82$ & $198.52 \pm 3.96$ \\
\hline$p$-value & 0.0038 & $<0.0001$ & 0.0296 & $<0.0001$ & 0.1298 & 0.0022 \\
\hline
\end{tabular}

Total Fatty Acid Content in Adipocytes. Differentiated adipocytes (with or without SCD1 $1_{\text {inhib }}$ ) were treated with $250 \mu \mathrm{M}$ PA, PMA, SA or OA for 48 hours. One-way ANOVA and Fisher's Least Significant Difference post-hoc test was used to assess for statistically significant differences between conditions. * Indicates a significant difference compared to the $\mathrm{D}+\mathrm{EtOH}$ control $(\mathrm{p}<0.05)$. $\mathrm{z}$ Indicates a significant difference compared to the $\mathrm{I}+\mathrm{EtOH}$ condition $(\mathrm{p}<0.05)$. Presented $\mathrm{p}$-values correspond to the pairwise comparison of D and I conditions for each FA treatment. Other FAs corresponds to the sum of all other FAs measured by gas chromatography, excluding PA, PMA, SA and OA. SCD1, stearoyl-CoA desaturase 1; FAs, fatty acids; D, dimethylsulfoxide; I, SCD1 inhibitor; PA, palmitate; PMA, palmitoleate; SA, stearate; OA, oleate; EtOH, ethanol. 
treatment ( $\mathrm{p}<0.001$, Figure 5.1). Elovl6 gene expression was significantly up-regulated 1.2-fold in cells treated with $\mathrm{I}+\mathrm{PA}$ relative to $\mathrm{I}+\mathrm{EtOH}(\mathrm{p}=0.03)$, which is notable given that Elovl6

expression was already up-regulated 2.4-fold with $\mathrm{I}+\mathrm{EtOH}$ treatment relative to $\mathrm{D}+\mathrm{EtOH}$ $(\mathrm{p}<0.001)$. ELOVL6 protein content was unaltered by I+PA treatment in adipocytes.

Treating adipocytes with MUFAs did not influence adipocyte FA profiles to the same extent as SFAs. Specifically, the D+PMA treatment did not significantly alter the levels of PA, $\mathrm{SA}$ or $\mathrm{OA}$, and the D+OA treatment did not alter PMA or SA content. However, there was a slight 1.2-fold increase in PA content in cells treated with D+OA. Similar results were seen with MUFA treatments in SCD1-inhibited adipocytes, where the I+PMA treatment did not alter PA, SA or OA content (Table 5.1), and the I+OA treatment did not alter PMA or SA content but did cause a slight 1.2-fold increase in PA levels. The D-PMA treatment also caused a 1.6-fold increase in vaccenic acid (VA) levels $(\mathrm{p}<0.001)$ (data not shown). Moreover, I+PMA increased VA levels significantly more than D+PMA (3.7-fold; $\mathrm{p}<0.001)$. These results reflect the increase in ELOVL6 activity seen in SCD1-inhibited adipocytes. However, I+PMA did not appear to influence Elovl6 gene expression or protein content (data not shown). Treating SCD1-inhibited adipocytes with either MUFA decreased $S c d 1$ gene expression (1.5-fold decrease with I+PMA $(\mathrm{p}=0.04)$ and 1.6-fold decrease with I+OA $(\mathrm{p}=0.002)) . S c d 2$ expression was slightly reduced by I+PMA treatment $(1.2$-fold, $\mathrm{p}=0.002)$, but not with I+OA. Protein content of SCD1 and ELOVL6 was unaltered by MUFAs in SCD1-inhibited adipocytes. 


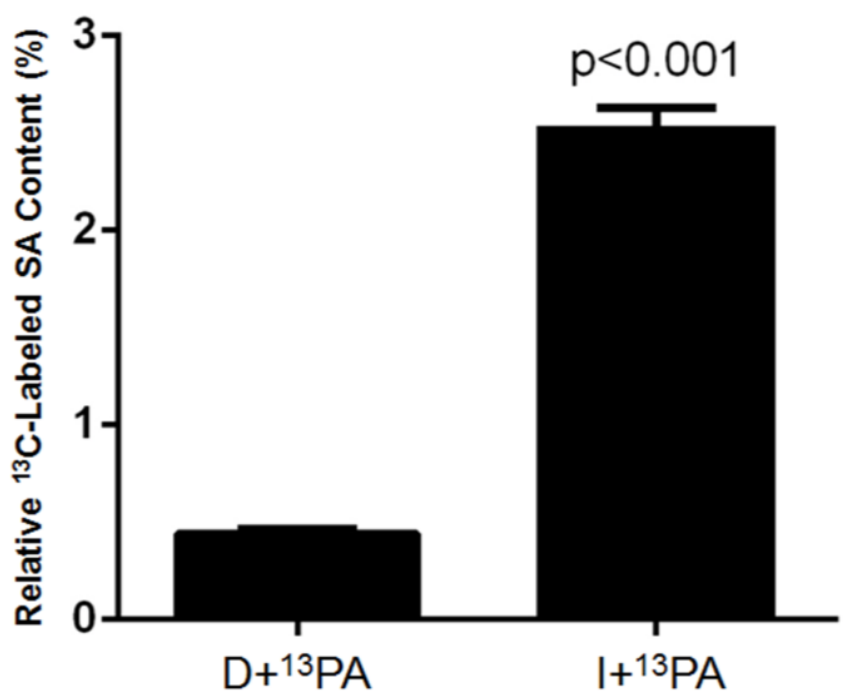

Figure 5.1. Relative ${ }^{13} \mathrm{SA}$ Content after $\mathrm{U}_{-}{ }^{13} \mathrm{C}$-labeled PA Treatment. Control and SCD1inhibited adipocytes were treated with $250 \mu \mathrm{M} \mathrm{U}-{ }^{13} \mathrm{C}$-labeled PA for 48 hours. Statistical significance between the conditions was determined used a two-tailed student's t-test. SCD1, stearoyl-CoA desaturase 1; D, dimethylsulfoxide; I, SCD1 inhibitor; ${ }^{13} \mathrm{PA}, \mathrm{U}-{ }^{13} \mathrm{C}$-labeled palmitate; ${ }^{13} \mathrm{SA},{ }^{13} \mathrm{C}$-labeled stearate.

\subsubsection{SFA and MUFA Treatments do not Influence Cellular Stress in SCD1-Inhibited Adipocytes}

While SFAs such as PA and SA have been reported to activate markers of cellular stress $[165,215,224]$, it is unknown if their effects would be amplified in SCD1-inhibited adipocytes. Furthermore, the effects of MUFAs on cellular stress in SCD1-inhibited adipocytes are unknown. FA treatments in control cells (i.e., D+PA, D+PMA, D+SA and D+OA) did not alter levels of phosphorylated JNK, STAT3, ERK1/2 or p38 MAPK (Figures 5.2-5.3). Treating adipocytes with either I+PA or I+PMA did not influence JNK, p38 MAPK, STAT3 or ERK1/2 activity (Figure 5.2). We also found no evidence that these stress kinases were altered in adipocytes treated with $\mathrm{I}+\mathrm{SA}$ and $\mathrm{I}+\mathrm{OA}$, although there did appear to be a slight, albeit statistically insignificant, increase in p-JNK and p-p38 MAPK with I+SA (Figure 5.3). 

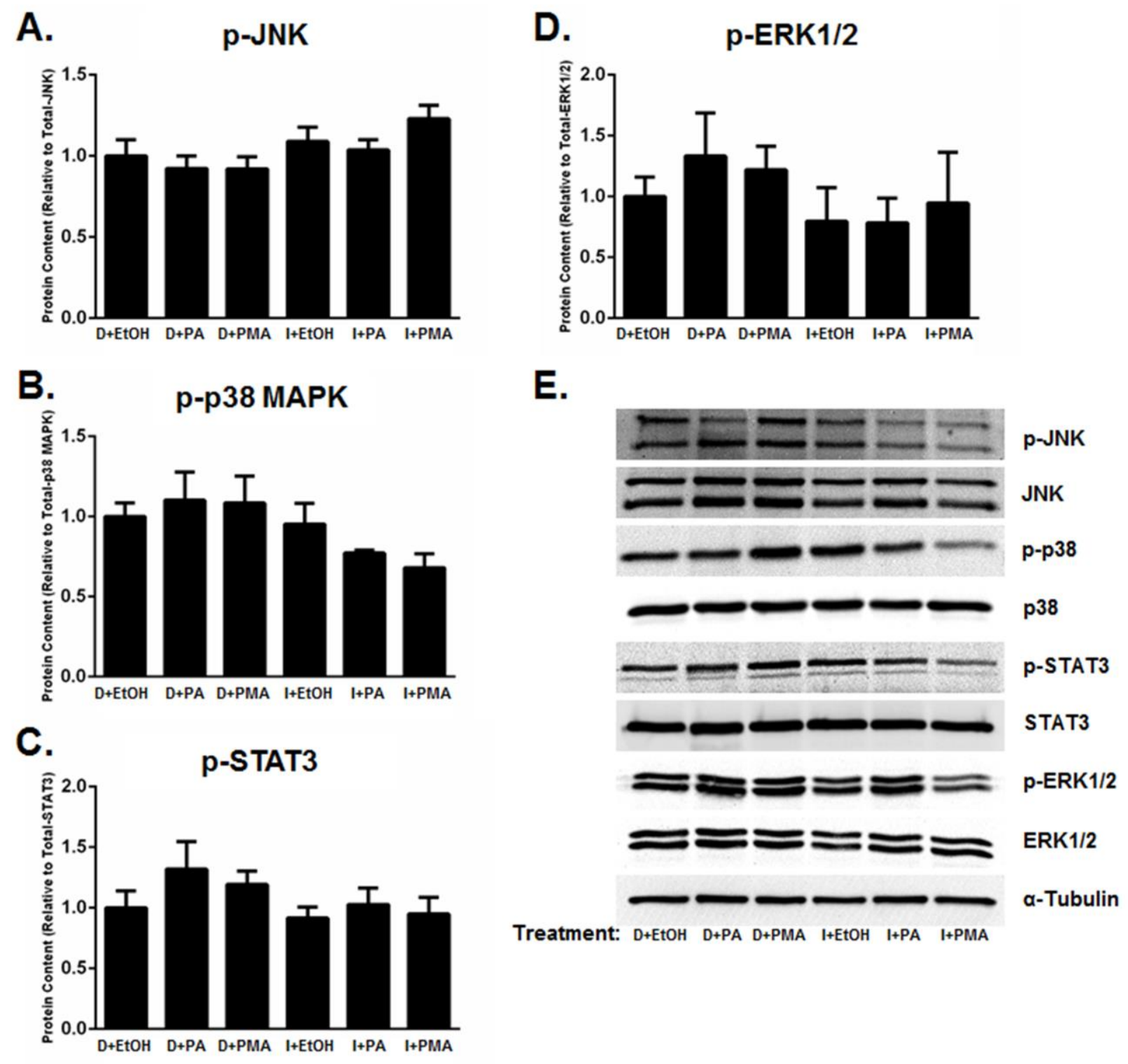

Treatment: D+EtOH D+PA D+PMA I+EtOH I+PA I+PMA

Figure 5.2. Markers of Cellular Stress in Adipocytes Treated with PA and PMA. Differentiated adipocytes (with or without $\mathrm{SCD} 1_{\text {inhib }}$ ) were treated with either $250 \mu \mathrm{M}$ PA or PMA for 48 hours. Western blotting was used to assess the protein content of (A) p-JNK, (B) p-p38 MAPK, (C) pSTAT3, (D) p-ERK1/2. Phosphorylated proteins are expressed relative to their total protein counterpart. Representative blots are shown in (E). JNK, jun N-terminal kinase; p38 MAPK, p38 mitogen-activated protein kinase; STAT3, signal transducer and activator of transcription 3; ERK1/2, extracellular signal-regulated kinases; D, dimethylsulfoxide; I, SCD1 inhibitor; EtOH, ethanol; PA, palmitate; PMA, palmitoleate. 
A.

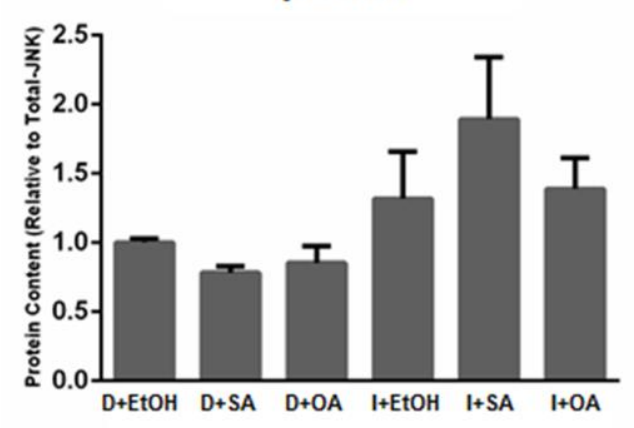

B.

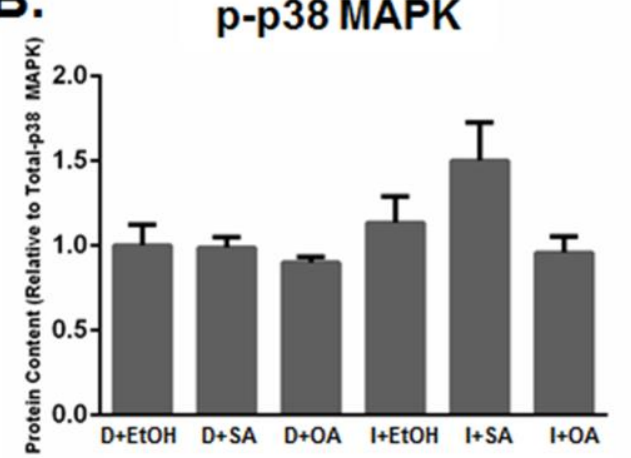

C.

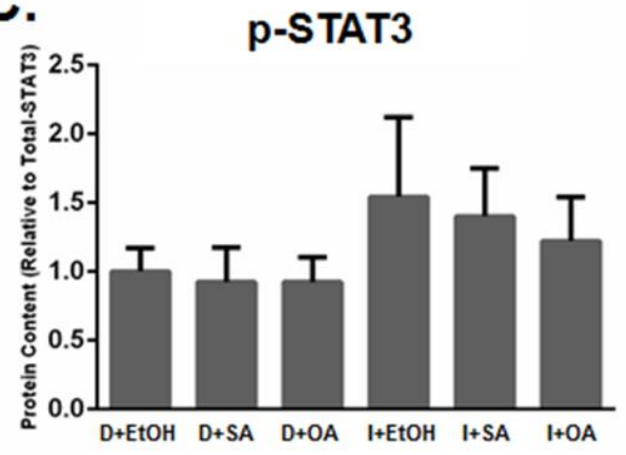

D.

p-ERK1/2

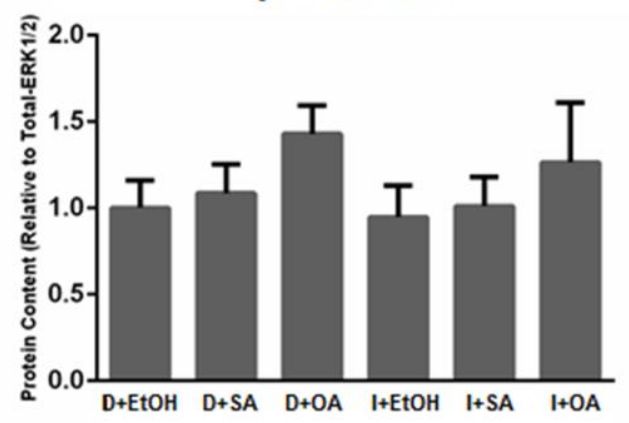

E.
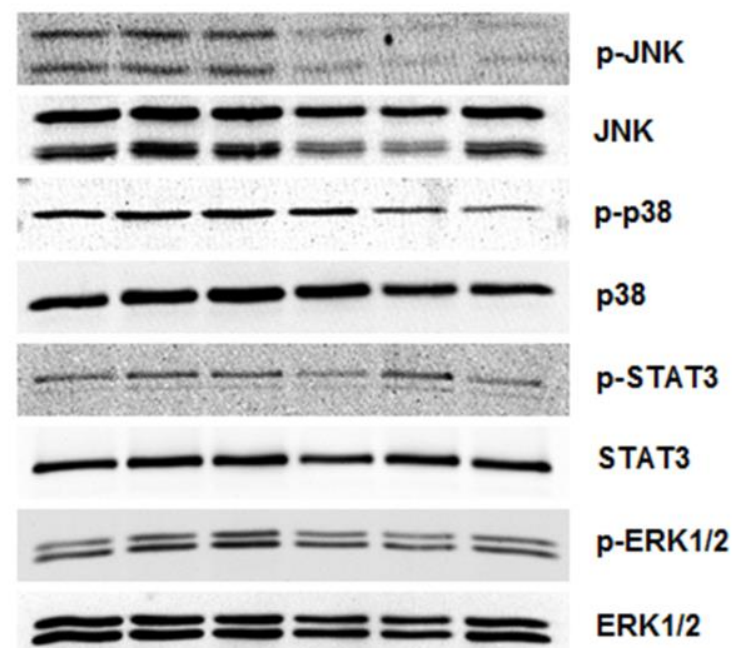

ERK1/2

a-Tubulin

Treatment: $D+E$ tOH $\quad D+S A \quad D+O A \quad$ I+EtOH $\quad$ I+SA $\quad$ I+OA

Figure 5.3. Markers of Cellular Stress in Adipocytes Treated with SA and OA. Differentiated

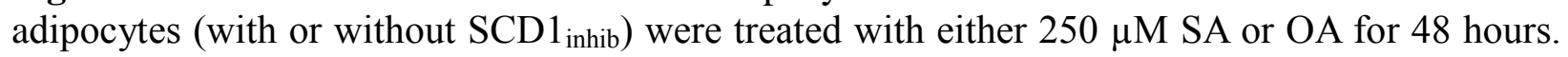
Western blotting was used to assess the protein content of (A) p-JNK, (B) p-p38 MAPK, (C) pSTAT3, (D) p-ERK1/2. Phosphorylated proteins are expressed relative to their total protein counterpart. Representative blots are shown in (E). JNK, jun N-terminal kinase; p38 MAPK, p38 mitogen-activated protein kinase; STAT3, signal transducer and activator of transcription 3; ERK1/2, extracellular signal-regulated kinases; D, dimethylsulfoxide; I, SCD1 inhibitor; EtOH, ethanol; SA, stearate; OA, oleate. 


\subsubsection{SCD1 mediates SFA and MUFA regulation of Cytokine Expression and Secretion}

FAs are known to influence adipocyte inflammatory signalling pathways [192], and previous work has also shown that SCD1 can regulate inflammatory gene expression in 3T3-L1 adipocytes [162]. We therefore examined if the effects of SFAs and MUFAs on gene expression and protein secretion of common inflammatory cytokines were exacerbated in SCD1-inhibited adipocytes.

D+PA and D+PMA treatments did not alter Ccl5 gene expression; however, treating adipocytes with SA and OA up-regulated $C c l 5$ gene expression: D+SA - 2.1-fold ( $\mathrm{p}=0.003)$ and D+OA - 1.5-fold ( $\mathrm{p}<0.001)$. Conversely, $M c p-1$ gene expression was slightly reduced by D+PA (1.2-fold; $\mathrm{p}=0.04)$, but significantly increased by D+SA (1.4-fold; $\mathrm{p}=0.01)$. MUFA treatments did not alter Mcp-1 gene expression in control adipocytes. Il-6 expression was under the detection limits of qRT-PCR. None of the SFA treatments affected the secretion of CCL5, MCP1, or IL-6 in control adipocytes (Figure 5.4).

In SCD1-inhibited adipocytes, gene expression of $C c l 5$ and $M c p-1$ was up-regulated 1.9fold $(\mathrm{p}=0.005)$ and 1.4 -fold $(\mathrm{p}=0.006)$ with $\mathrm{I}+\mathrm{EtOH}$ treatment. Upon examining cytokine secretion, CCL5, but not IL-6 or MCP-1, was increased 2.1-fold ( $\mathrm{p}<0.001)$ with I+EtOH treatment compared to D+EtOH (Figure 5.4). While SCD1-inhibition alone did not have a major impact on inflammatory cytokine expression or secretion, treating SCD1-inhibited adipocytes with SA had more profound effects. Specifically, the I+SA treatment caused a significant 3.2fold $(\mathrm{p}=0.04)$ increase in $M c p-1$ and a 5.3-fold $(\mathrm{p}=0.001)$ increase in $C c l 5$ expression compared to I+EtOH. Il-6 expression was under the detection limits of qRT-PCR. I+SA treatment also caused a significant 17.8 -fold $(\mathrm{p}<0.001)$ and 4.0-fold $(\mathrm{p}<0.001)$ increase in IL-6 and MCP-1 
protein secretion compared to the I+EtOH condition (Figure 5.4); however, I+SA did not alter CCL5 secretion.

A.

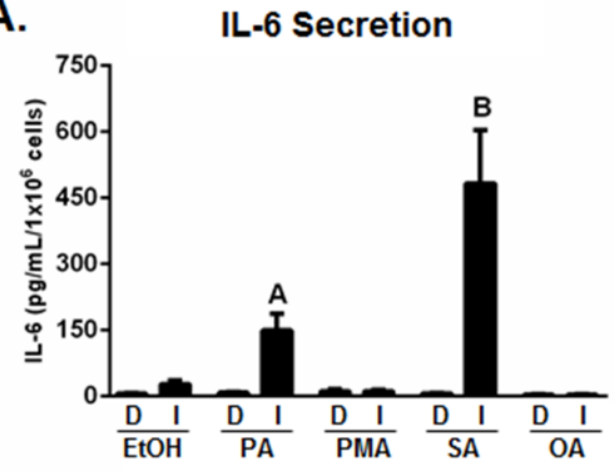

B.

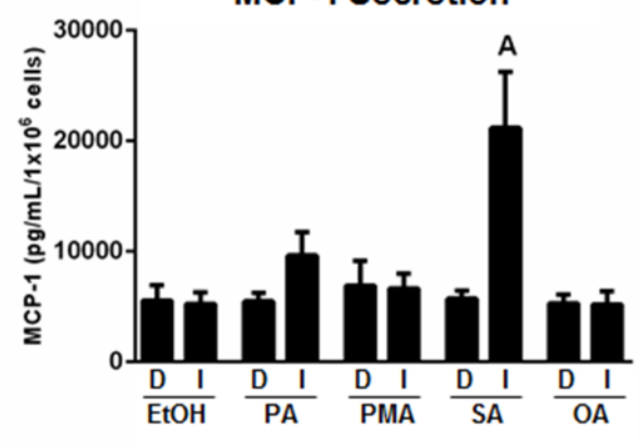

C.

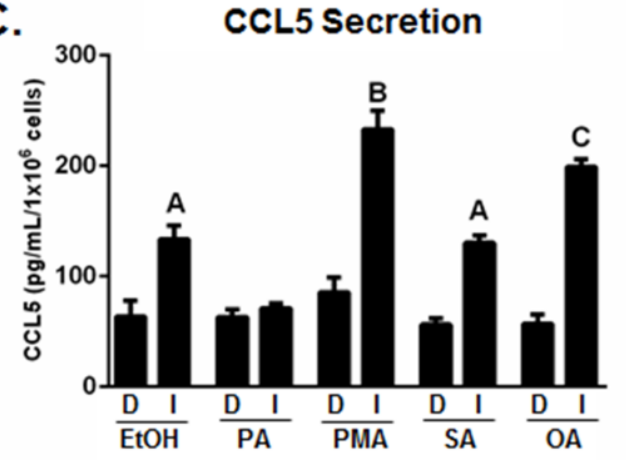

Figure 5.4. Cytokines Secreted from Adipocytes Treated with FAs. Differentiated adipocytes (with or without $\mathrm{SCD} 1_{\text {inhib }}$ ) were treated with either $250 \mu \mathrm{M}$ PA, PMA, SA or OA for 48 hours. Culture media was assessed for secreted (A) IL-6, (B) MCP-1 and (C) CCL5. One-way ANOVA and Fisher's Least Significant Difference posthoc test was used to assess statistical differences between conditions. Data bars not sharing an identical letter are statistically different $(p<0.05)$. Secreted IL-6 from the I+PA condition was borderline significantly different from the control condition $(p=0.06) . \quad$ IL-6, interleukin-6; MCP-1, monocyte chemotactic protein-1; CCL5, chemokine (C-C motif) ligand 5; D, dimethylsulfoxide; I, SCD1 inhibitor; EtOH, ethanol; PA, palmitate; PMA, palmitoleate; $\mathrm{SA}$, stearate; OA, oleate. 
Of the other FA treatments in SCD1-inhibited adipocytes, I+PA and I+OA significantly increased $M c p-1$ gene expression 1.2-fold ( $\mathrm{p}=0.01)$ and 1.4-fold ( $\mathrm{p}=0.03)$, respectively; however, no other FA treatments increased adipocyte MCP-1 secretion. Ccl5 gene expression was upregulated in cells treated with I+PA $(2.7$-fold; $\mathrm{p}=0.02)$, unaltered by I+PMA, and decreased with I+OA (1.4-fold; $p=0.006$ ) (data not shown). In contrast, CCL5 secretion appeared to be lower for I+PA compared to I+EtOH, but increased for I+PMA and I+OA (Figure 5.4).

\subsection{Discussion}

FAs are capable of influencing a myriad of biological processes in AT including inflammatory, metabolic, and insulin signalling pathways [140, 225]. Furthermore, previous work has shown that distinct FAs have different effects on the transcriptional profile of adipocytes [64]. Given that the most abundant FAs in AT are linked to SCD1 as either enzyme substrates (i.e., PA and SA) or products (i.e., PMA and OA) [118, 125, 202, 216], it is important to assess the extent to which SCD1 plays a role in mediating their bioactivity. Therefore, our goal was to study if the influence of FAs on adipocyte inflammatory status, cellular stress, and FA profiles is altered when SCD1 activity is compromised.

5.5.1 SCD1-Inhibited Adipocytes Effectively Take up Exogenous FAs, and Up-Regulate PA Elongation to $S A$

As expected, FA treatments caused a significant increase in the abundance of the corresponding FAs within adipocytes, regardless of SCD1 inhibition. FAs taken up by adipocytes are normally incorporated into TAG molecules for storage; however, SCD1 inhibition is known to decrease total TAG content both at the tissue level [216, 226] and adipocytes [201]. 
Therefore, despite a compromise in TAG production in SCD1-inhibited adipocytes, it appears as though these cells are still able to take up FAs. This suggests that the FAs are being incorporated into other lipid adipocyte lipid fractions, such as free fatty acids (FFAs), diacylglycerols, and cholesteryl esters. This is supported by previous work from our group showing that FFA, diacylglyerols and cholesteryl ester levels are increased in SCD1-inhibited adipocytes [201].

Treating SCD1-inhibited adipocytes with PA (i.e., I+PA) caused an increase in cellular SA levels, which prompted us to examine elongation using a stable isotope tracer. We demonstrated that when SCD1 activity is inhibited, adipocyte ELOVL6 activity is increased. While previous work from our laboratory showed that Elovl6 gene expression was up-regulated in SCD1-inhibited 3T3-L1 adipocytes [201], this is the first time that this has been demonstrated using a stable isotope tracer. We initially surmised that this increase in ELOVL6 activity may occur in order to mitigate the cellular accumulation of PA, which is purported to promote cellular stress and inflammation [201, 227]; however, the results of our analysis of stress markers and cytokine secretion did not support this hypothesis.

\subsubsection{SCD1 Inhibition and Exogenous FAs do not Alter Makers of Cellular Stress}

Certain stress stimuli (e.g., apoptosis, cytokines and ligands for toll-like receptors) can be increased by FAs and, in turn, can induce intracellular signalling pathways including the jun $\mathrm{N}$ terminal kinase (JNK) system, extracellular signal-regulated kinases (ERK1/2), p38 mitogenactivated protein kinase (MAPK), as well as signal transducer and activator of transcription 3 (STAT3) [228-232]. However, we found no evidence that FAs altered the activation of JNK, ERK1/2, p38 MAPK or STAT3 in SCD1-inhibited adipocytes compared to controls. The lack of cellular stress in SCD1-inhibited adipocytes treated with SFAs was unexpected, since SFAs have 
previously been reported to induce the aforementioned signalling cascades [165, 215, 224]. For instance Guo et al. showed that ERK1/2 and JNK were activated in 3T3-L1 preadipocytes treated with $250 \mu \mathrm{M} \mathrm{PA}$, but not OA, [215]; however, these authors conducted their experiments in undifferentiated preadipocytes, while we used differentiated adipocytes. The use of undifferentiated preadipocytes by Guo et al. is a notable difference with our work because it was previously postulated that preadipocytes may control p-ERK levels in order for adipogenesis to occur [230]. Since our studies were conducted in differentiated adipocytes, this may explain why we did not see a change in p-ERK levels. Koeberle et al. also showed that inhibiting SCD1 in murine NIH-3T3 fibroblasts did not influence ERK1/2 or JNK activation [165].

FA doses between $50 \mu \mathrm{M}$ and $500 \mu \mathrm{M}$ are considered well within the physiologically relevant range for both human and rodent models [192, 233, 234]. Our moderate FA dose of 250 $\mu \mathrm{M}$ may provide one possible explanation for the discrepancies between our results and those of previous studies. For instance, work by Yin et al. showed that PA treatment in 3T3-L1 adipocytes significantly increased p-JNK protein levels [235]. However, Yin et al. observed these effects using a PA dose of $500 \mu \mathrm{M}$; whereas in our study $250 \mu \mathrm{M}$ PA did not alter p-JNK levels. This suggests that a higher dose of PA may be necessary in order to induce JNK signalling in adipocytes. Differences in FA treatments may also explain discrepancies between our findings regarding p38 MAPK and those reported by Koeberle et al. [165]. While we observed no effect with $250 \mu \mathrm{M}$ FA treatments on p38 MAPK activation in SCD1-inhibited adipocytes, Koeberle et al. reported that higher doses (i.e., $400 \mu \mathrm{M}$ ) of SFAs activated p38 MAPK in NIH-3T3 cells [165]. Finally, the fact that we did not see a change in p-STAT3 levels in SCD1-inhibited adipocytes was not entirely surprising since STAT3 is downstream of p38 MAPK, JNK or ERK1/2 [236, 237]. 
5.5.3 The Effects of Exogenous FAs on Inflammatory Markers are Exacerbated in SCD1Inhibited Adipocytes

There does not appear to be a consensus regarding the anti- or pro-inflammatory effect of SCD1 in AT or adipocytes [18]. While there are reports of SCD1 inhibition promoting inflammation despite improvements in metabolic disease [18], previous work has also shown that reduced SCD1 can prevent adipocyte inflammation [120]. Our results add to the existing body of knowledge and suggest that inhibiting SCD1 may have pro-inflammatory consequences in 3T3-L1 adipocytes. Further, since FAs are known to modulate adipocyte inflammation [192], we were also interested in exploring whether SCD1 can mediate FA-regulation of inflammation. Although none of the FA treatments altered the secretion of common markers of inflammation (i.e., IL-6, MCP-1, and CCL5) in control adipocytes, we found that the combination of SCD1 inhibition and SFA treatments caused a significant increase in the secretion of these inflammatory markers. Therefore, while normal adipocytes may be able to effectively "handle" FAs without triggering the production of inflammatory signals, this appears to be compromised when SCD1 activity is inhibited.

Of the four FAs used in the present investigation, SA evoked the greatest changes in inflammatory gene expression and protein secretion. Treating control adipocytes with SA increased the expression of $C c l 5$ and $M c p-1$; however, these effects were exacerbated in SCD1inhibited adipocytes. Further, this was evidenced at the level of both gene expression and cytokine secretion. While previous investigations have demonstrated that PA promotes MCP-1 and IL-6 secretion [238] and gene expression [239, 240] in 3T3-L1 adipocytes, these prior studies did not examine the effects of SA. Schaeffler et al. conducted one of the few studies 
examining the influence of SA on cytokine secretion from adipocytes, and they found that 100 $\mu \mathrm{M}$ SA induced MCP-1 secretion, but to a lesser extent than $100 \mu \mathrm{M}$ PA [241]. While PA and SA are both saturated FAs, we have previously shown that they trigger distinct transcriptional responses in adipocytes [64]. Our results suggest that when SCD1 activity is compromised in adipocytes, SA appears to have greater pro-inflammatory effects compared to PA. This may stem from an overload of SA, since the combination of SA treatment and increased production via ELOVL6 contribute to SA levels that are $~ 17$-fold greater than those of control adipocytes.

We acknowledge the limitation that we have only targeted SCD1 in our analyses despite the fact that adipocytes also express SCD2. However, SCD1 is the dominant isoform in AT [28, 66]. We are also unable to translate our results from adipocytes to AT, since other cell-types residing in AT (e.g. macrophages, T-cells, endothelial cells etc.) may also have important roles propagating the relationships between FAs and inflammation [140]. However, based on our observations regarding inflammatory protein secretions from SCD1-inhibited adipocytes, future work should aim to examine how these secretions may influence the function of other cell-types in AT. Finally, future work should consider examining various FA concentrations in order to assess if cellular stress responses are exacerbated with higher FA doses.

\subsubsection{Summary and Perspectives}

The present study has provided novel information regarding how SCD1 mediates the effects of SFA and MUFA treatments in adipocytes. FAs had different effects on adipocyte lipid profiles, as well as inflammatory cytokine gene expression and secretion, when SCD1 activity was compromised. Specifically, we showed that the influence of FAs on inflammatory markers is exacerbated when adipocyte SCD1 activity is inhibited. In addition, SA appears to be more 
pro-inflammatory than PA in SCD1-inhibited adipocytes, as evidenced by larger increases in inflammatory cytokine secretion and expression. We have also demonstrated that PA elongation to SA is up-regulated in adipocytes when SCD1 activity is compromised. These results not only highlight the diverse effects of individual FAs, but advance our knowledge regarding how SCD1 can mediate the bioactivity of FAs in adipocytes. 


\section{CHAPTER 6:}

INTEGRATIVE DISCUSSION 


\section{$\underline{\text { 6.1 Study Summaries }}$}

To gain a better understanding of how altered SCD1 activity can influence adipocyte metabolism, this thesis examined several aspects of adipocyte biology using a model of SCD1 inhibition. Specifically, this thesis assessed changes in lipid biosynthesis and metabolism, global gene expression, basal insulin signaling, inflammation and cellular stress, in an attempt to better understand the roles and influences of SCD1 in adipocytes. The presented results emphasize the wide-ranging impact of SCD1 on the production of specific FAs and lipids within adipocytes. Additionally, the results from this thesis revealed that SCD1 considerably modified the effects of exogenous FAs within adipocytes.

\section{$\underline{6.1 .1 \text { Chapter } 3 \text { Summary }}$}

Prior to the research conducted in this thesis, the full extent of changes in gene expression and lipid content elicited by reduced SCD1 activity in adipocytes was unclear. The first study presented in this thesis examined SCD1-inhibited 3T3-L1 adipocytes for changes in gene expression, protein content and total FAs. This study confirmed that the SCD1 inhibitor (used in all the studies presented in this thesis) specifically targeted SCD1 enzyme activity and not Scdl gene expression or SCD1 protein content. Importantly, this enabled the specific assessment of altered SCD1 activity in the adipocytes. This study has built upon the results seen in Scd1-/mice (e.g., reduced whole-body TAGs) by confirming a parallel change in SCD1-inhibited adipocytes, and has additionally revealed a complex gene network that likely contributed to the decrease in TAGs. 


\subsubsection{Chapter 4 Summary}

The full extent to which SCD1 inhibition influences the content and composition of lipid fractions (PLs, TAGs, CEs, DAGs, and FFAs) was unknown prior to the research conducted in the second study of this thesis. This is important, since changes in the abundance or composition of these lipid fractions can dramatically alter adipocyte metabolism [125, 208]. This second study advanced our knowledge regarding the widespread effects of SCD1 on adipocyte lipid profiles, and notably revealed that SCD1 inhibition in adipocytes increased the SFA:MUFA ratio in PLs, increased cellular DAGs, and caused partial impairments in basal insulin signaling.

\subsubsection{Chapter 5 Summary}

The final study of this thesis illustrated the influence of SCD1 as a mediator of the effects of FAs within adipocytes. Control adipocytes (with a functional SCD1 enzyme) were able to tolerate exogenous FAs (i.e., PA, PMA, SA and OA) without the induction of inflammatory markers or cellular stress, but this was not the case in SCD1-inhibited adipocytes. Specifically, SCD1 inhibition led to SFAs eliciting greater increases in inflammatory markers compared to their effects in control adipocytes. Further, SA elicited greater increases in inflammatory markers compared to PA, emphasizing the ability of distinct FAs to differentially influence adipocyte metabolism. Finally, this study confirmed for the first time that 3T3-L1 adipocytes with compromised SCD1 activity up-regulated the elongation of PA to SA, which could have significant ramifications for adipocyte metabolism. 
Collectively, the results presented throughout this thesis have illustrated several important influences of SCD1 activity in adipocytes. The forthcoming sections will discuss some of these influences in a larger context.

\subsection{SCD1 Activity has Protective Influences in Adipocytes}

Although results in $S c d 1 /-$ mice suggest that a reduction in SCD1 activity can induce whole-body benefits (e.g., increased insulin sensitivity and protection from diet-induced obesity) [15], this thesis has provided evidence that reduced SCD1 activity in adipocytes may have caused detrimental effects (e.g., reduced insulin signaling, increased inflammatory cytokine expression and cellular DAG accumulation). Furthermore, these results suggest that normal SCD1 activity in adipocytes can elicit numerous protective effects, as follows:

\subsubsection{Ability to Tolerate Exogenous FAs}

One of the protective influences of SCD1 activity may involve the adipocytes' ability to handle exogenous FAs without inducing inflammation. The results in this thesis demonstrate that adipocytes appear to tolerate moderate levels of exogenous FAs (i.e., $250 \mu \mathrm{M}$ for 48 hours) when SCD1 activity is uncompromised. However, the inhibition of SCD1 activity led adipocytes to experience a stronger inflammatory response to certain FA treatments. Specifically, SCD1inhibited adipocytes had substantial increases in common inflammatory markers when treated with exogenous SA. SA content in the AT of human adults is generally around $3 \%$ of the total AT FA pool $[242,243]$. However, the results from this thesis showed that the combination of exogenous SA (from FA treatments) and endogenously synthesized SA (via up-regulated 
ELOVL6 activity) promoted total SA levels of almost 25\% in SCD1-inhibited adipocytes. Together, these results may suggest that SCD1 activity helps prevent 'SA overload' in adipocytes (i.e., SA levels far beyond what would normally be seen in AT). This may have significant implications for adipocytes, since SA has been associated with the induction of inflammation and cell death in human aortic endothelial cells [244]. Anderson et al. showed that treating mouse peritoneal macrophages with SA activated inflammatory signaling (via TLR-4/2) which caused ER stress [245]. Therefore, a reduced capacity of SCD1-inhibited adipocytes to store FAs within TAGs (which was partially supported by the observed decrease in TAG content in this thesis), may contribute to the inability of these adipocytes to effectively handle exogenous proinflammatory FAs such as SA without inducing inflammatory markers.

\subsubsection{The Prevention of DAG Accumulation and Impairments in Insulin Signaling}

Studies in adipocyte models have shown us that reduced SCD1 activity can cause detrimental effects to adipocyte biology (e.g., inflammation), which suggests that SCD1 activity has protective influences in adipocytes. For instance, studies have demonstrated that SCD1 inhibition in 3T3-L1 adipocytes reduced adiponectin expression [27], increased cell death [112], and induced ER stress [165]. This thesis has added to this list of potential detrimental outcomes of reduced SCD1 activity, and has shown that SCD1 inhibition increased total cellular DAG composed primarily of SFAs (Figure 6.1) [201, 246]. Increased cellular DAGs have been previously associated with the inhibition of the insulin signaling pathway in skeletal muscle of humans and rodents [207, 208, 247]. Holloway et al. showed that reductions in SA content within DAGs were associated with contraction-induced improvements in insulin sensitivity of red muscle in obese Zucker rats [247]. In line with this, the results presented in Chapters 3-5 
have provided evidence that SCD1 inhibition caused an accumulation in DAG-SFAs (particularly DAG-SA), that may underlie the impairments in basal insulin signaling (Chapter 4), and the down-regulation of the insulin signaling pathway (Chapter 3). However, markers of cellular stress were unaltered (Chapters 4 and 5) [201, 246]. Results from Chapter 4 further suggest that the increase in DAGs may stem from changes in gene expression (e.g., down-regulation of $D g k \delta$ ) that occurred in SCD1-inhibited adipocytes. However, these results cannot confirm if insulin signaling is directly influenced by the increase in DAG-SFAs, or if other factors (e.g., increased ceramides, altered cell membrane fluidity) contributed to this outcome. PA, which accumulated in SCD1-inhibited adipocytes (Chapters 3-5) is a substrate for ceramide synthesis [208], and ceramides have been documented to inhibit Akt phosphorylation in myotubes [248]. Although ceramides were not measured in this thesis research, the observed impairments in Akt phosphorylation underline that examining reactive lipid species in more detail will be interesting in future SCD1 studies in adipocytes.

It should be noted that our results regarding insulin signaling and inflammatory markers conflict with findings from another study that reported reduced inflammation in adipocytes from Scd1-/- mice [120]. However, results observed in adipocytes from Scd1-/- mice can be confounded by compensatory effects in other tissues that occurred throughout the lifespan of the mice (prior to adipocyte isolation), and may have altered adipocyte metabolism through mechanisms including tissue cross-talk. For instance, elevations in skeletal muscle insulin signaling [195], as well as FA oxidation in the liver and skeletal muscle of $S c d 1-/$ - mice [15, 28], could all contribute to: 1) reduced accumulation of AT mass, 2) altered systemic cytokine levels, and 3) improvements in AT insulin sensitivity and inflammatory status. If these metabolic changes occur prior to adipocyte isolation, this could influence the results of studies examining 
adipocytes from Scd1-/- mice. Our findings have therefore added important information regarding insulin signaling and DAG-SFA accumulation in adipocytes with compromised SCD1 activity.

\subsubsection{Production of a Potential Lipokine}

Additional rationale for a protective role of SCD1 in adipocytes lies in its ability to synthesize PMA, a FA that may have an important role in metabolic signaling. Specifically, PMA and its related metabolites have been designated as 'lipokines', which are lipid signaling molecules that are actively secreted from adipocytes or AT and could have endocrine functions $[13,164]$. The potential for PMA to act as a lipokine is a relatively novel notion (i.e., within the last five years) that has not been thoroughly investigated, but stems from several points of justification. Firstly, PMA content is the fifth highest of all FAs within blood lipids and lipogenic tissues including AT, yet the dietary intake of PMA in the average human adult is low (i.e., approximately $1-2 \%$ of total daily fat intake) [249]. Therefore changes in endogenous PMA synthesis (via altered SCD1 activity) have the potential to cause dramatic spikes in PMA content within lipogenic tissues. The results presented in Chapters 3 and 4 support the potential for altered PMA content to signal a change in the rate of de novo lipogenesis. Specifically, PMA content was observed to be very low prior to the onset of lipogenesis in adipocytes, but increased dramatically throughout adipocyte differentiation [246]; however, the increase in PMA content was completely blunted by SCD1 inhibition. Although speculative, these results may support the potential of PMA levels to signal the onset of de novo lipogenesis and TAG production. Additionally, Chapter 5 examined how exogenous PMA may influence adipocyte biology, and although PMA did not elicit changes in cytokine secretion or cellular stress, I+PMA was the only 
condition to significantly reduce the expression of Ppary, and D+PMA induced a non-significant decrease in Ppary (data not shown). If PMA content acts as a signal of lipogenesis, then this may explain why supplementing adipocytes with exogenous PMA reduced Ppary. The results of this thesis are therefore in support of the ability of PMA to alter (or potentially signal) a change in lipogenesis.

A second justification for the ability of PMA to act as a lipokine stems from positive associations between plasma PMA levels and insulin sensitivity in humans [249]. Mice infused with PMA also display enhanced whole-body glucose homeostasis, thus supporting a potential insulin-sensitizing role for PMA [13]. The results in this thesis support this notion, since PMA content was significantly blunted in SCD1-inhibited adipocytes, which coincided with impairments in insulin signaling. Taken together, these results support a protective role of SCD1

activity in synthesizing PMA levels, which in turn may help preserve insulin sensitivity and could function as a signal of the onset of lipogenesis in adipocytes.

Collectively, the results presented in this thesis suggest that SCD1 activity has important protective influences in adipocytes by 1) enhancing the ability of adipocytes to handle exogenous FAs without inductions in inflammation, 2) preventing DAG-SFA accumulation and impairments in insulin signaling and 3) producing high levels of PMA in adipocytes, which may have an insulin-sensitizing role.

\subsection{Altered Lipid Metabolism in SCD1-Inhibited Adipocytes}

In addition to eliciting effects that may have protective influences in adipocytes, this thesis has uncovered several notable changes in lipid metabolism that merit further discussion. 


\subsubsection{Altered Triacylglycerol Biosynthesis}

Results from this thesis have demonstrated that SCD1 inhibition caused a considerable reduction in adipocyte TAG content that stemmed primarily from a decrease in TAG-MUFAs produced via SCD1 (Figure 6.1). These results align with observations from Scd1-/- mice, which have reduced TAG and MUFA content in the skin, eyes, liver and AT [29, 30]. To date, SCD1 research has identified two key mechanisms to explain the link between reduced SCD1 activity and TAG content in tissues of humans and rodents. Firstly, SCD1-derived MUFAs are the predominant constituents of TAGs within rodent and human liver and AT [11, 16], and therefore a reduction in MUFA production via decreased SCD1 activity would lead to lower TAG levels. Secondly, SCD1 and DGAT2 have been observed to co-localize on the ER of HeLa cells, which has prompted the hypothesis that SCD1 facilitates the channeling of FAs towards DGAT2 for TAG synthesis due to the proximity of these enzymes within the cell [101]. Results of this thesis have contributed to these lines of thinking by 1) confirming that levels of TAG-MUFAs (produced via SCD1) could also be reduced in SCD1-inhibited adipocytes (extending the observations from prior studies into an adipocyte model), and 2) showing that SCD1-inhibited adipocytes down-regulated the expression of Dgat2 [201]. The reduction in Dgat2 expression aligns with observations by Collins et al., where primary human adipocytes with low SCD1 expression also had reduced $D G A T 2$ expression compared to adipocytes with higher $S C D 1$ levels [125]. Future studies assessing the links between SCD1 and DGAT2 in adipocytes should therefore not only confirm their ability to co-localize in this cell type, but should examine how SCD1 activity may alter Dgat2 transcription (e.g., if lower Dgat2 expression is merely a cellular adaptation to lower MUFA levels). 
In addition to providing information that supplements the current rationale linking SCD1 activity with TAG synthesis, this research has uncovered a series of biological pathways and genes that are altered by SCD1 inhibition in adipocytes, and may contribute to reduced TAG content [201]. Furthermore, the genes and pathways identified in this thesis (e.g., insulin signaling pathway, biosynthesis of unsaturated FAs, glycerolipid metabolism, adipocytokine signaling pathway) have provided new research avenues for SCD1 researchers. For instance, future studies should assess how SCD1 alters the expression of genes coding for enzymes of TAG biosynthesis (e.g., signaling mechanisms or transcriptional regulation of gene expression). In addition, future research should investigate whether a reduction in the ability of adipocytes to synthesize TAGs can induce detrimental effects on adipocyte biology. This is relevant to study since reduced TAG production in adipocytes could contribute to ectopic fat deposition in the liver and skeletal muscle $[125,250]$, and may therefore cause downstream metabolic complications at the whole-body level. For instance, results in this thesis suggest that SCD1 activity in adipocytes is necessary to prevent the induction of inflammatory markers by exogenous FAs. Furthermore, Caron-Jobin et al. have suggested that elevated SCD1 activity and fat storage within subcutaneous AT could be protective against metabolic disorders (e.g., insulin resistance). Taken together, these results may suggest that adipocytes require SCD1 activity to promote the storage of excess FAs into TAGs (e.g., TAG-MUFAs) to prevent cellular inflammation.

\subsubsection{Altered Phospholipid Fatty Acid Composition and the Maintenance of Oleate Levels}

SCD1 activity has been hypothesized to have a role in regulating the SFA:MUFA ratio within the cellular lipid membrane of epithelial cells, skeletal muscle and the Harderian gland 
$[10,39,251]$. By causing an accumulation of SFAs and a reduction of MUFAs, a decrease in SCD1 activity would be expected to increase the rigidity of the cell membrane, which in turn could influence cell signaling cascades (e.g., insulin signaling) [125]. This thesis has confirmed that SCD1 inhibition in 3T3-L1 adipocytes elevated the SFA:MUFA ratio in PLs (Figure 6.1), which was driven by significant increases in SFAs (PA and SA) and a very dramatic decrease in PMA content; however, OA content in SCD1-inhibited adipocytes was either maintained or slightly increased within the PL fraction. The increased PL SFA:MUFA ratio in SCD1-inhibited adipocytes may have contributed to the observed impairments in insulin signaling (i.e., reduced phosphorylation of $\mathrm{p}-\mathrm{Akt}^{\mathrm{Ser} 473}$ ) since this pathway is known to be influenced by membrane fluidity [125]. Future investigations could consider directly measuring cell membrane fluidity in SCD1-inhibited 3T3-L1 adipocytes, as well as analyzing additional proteins in the insulin signalling pathway (e.g., IRS-1 and GLUT4) and insulin-stimulated glucose uptake.

The maintenance of OA levels in PLs of SCD1-inhibited adipocytes may be explained by the apparent importance of OA for cell function. The significance of maintaining adequate OA levels within the cell membrane (where the majority of PLs are contained) has been demonstrated by several important findings. Firstly, investigations of model cell membranes, Chinese hamster ovary cells, and HepG2 cells all established that higher membrane OA content (and/or treatment with OA) was associated with increased cell membrane fluidity [252, 253]. Secondly, work using model membranes has shown that OA helped maintain PL spacing in the lipid bilayer, which also ensured an appropriate level of hydration in the membrane [252]. In addition to maintaining membrane fluidity, OA content has been proposed to facilitate the induction of hepatic lipogenesis $[26,62]$ and also serves as the main FA substrate for TAG production in the liver [62]. Finally, OA from the secretion media of cultured primary adipocytes 
from $S c d 1$-/- mice has been documented to reduce inflammation in macrophages and endothelial cells [120], suggesting an anti-inflammatory role of adipocyte-derived OA. Along these lines, Harvey et al. showed that OA treatment in human aortic endothelial cells displaced SA from the PL bilayer, which prevented SA-induced inflammation and cytotoxicity (i.e., cell death) [244]. Collectively, OA appears to have several important roles in the maintenance of proper cell function.

While the importance of OA has been well documented, this thesis has now demonstrated the apparent ability of SCD1-inhibited adipocytes to maintain total OA levels (as well as OA content within the PL fraction). Additionally, this thesis has shed light on how adipocytes may accomplish the feat of maintaining OA levels when SCD1 activity is compromised. For instance, the up-regulation of ELOVL6 activity may have served as a compensatory response of the SCD1-inhibited adipocytes (in an effort to maintain OA levels). OA is produced endogenously by elongation of $\mathrm{PA}$ and the subsequent desaturation to $\mathrm{OA}$, and therefore the substantial channeling of FAs towards SA synthesis would allow any residual SCD1 activity (beyond the inhibition induced by $\mathrm{SCD} 1_{\text {inhib }}$ ) or SCD2 activity (which shows substrate specificity towards SA) to desaturate SA into OA [53]. Furthermore, treating SCD1-inhibited adipocytes with exogenous OA (Chapter 5) caused a significant 1.4-fold decrease in Elovl6 expression. This suggests that up-regulated Elovl6 expression and activity in SCD1-inhibited adipocytes may be a means of maintaining OA levels, since the addition of exogenous OA reversed the effects on Elovl6 in SCD1-inhibited adipocytes. In agreement with the results of this thesis, Collins et al. have also observed increased channeling of PA towards elongation in human adipocytes treated with siRNA against $S C D 1$ [125]. Together, these results encourage the future assessment of how ELOVL6 and SCD1 activity may be connected within the context of OA synthesis and 
membrane fluidity. In particular, important insights could come from co-treating adipocytes with inhibitors or siRNA targeted towards Scd1 and/or Elovl6.

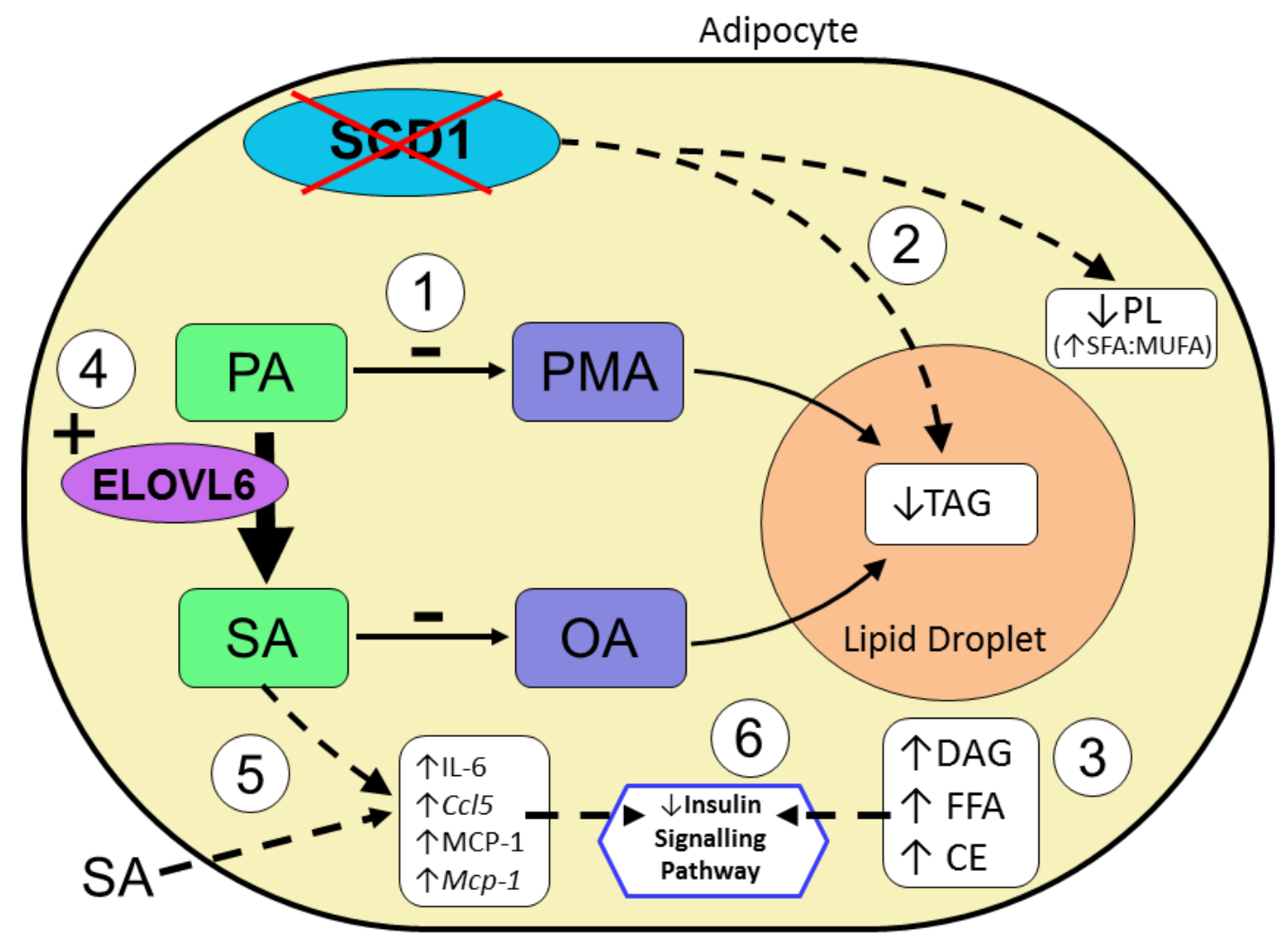

Figure 6.1. Integrated effects of SCD1 inhibition in adipocytes. Compromised SCD1 activity results in: (1) reduced endogenous synthesis of MUFAs PMA and OA; (2) a reduction in TAG and PL content, which coincides with the down-regulation of genes associated with the biosynthesis of these lipids and an increase in the SFA:MUFA ratio of membrane PLs; (3) increased DAG-SFAs, as well as total DAGs, FFAs and CEs; (4) up-regulation of ELOVL6 activity, which leads to increased production of SA; (5) the inability to effectively handle a combination of endogenous SA accumulation plus exogenous SA without elevations in inflammatory markers; (6) partial impairments in basal insulin signaling as well as the downregulation of the insulin signaling pathway, which could be due to either inflammatory factors or the increase in DAG-SFAs. 


\subsection{Prospective Research Possibilities and Remaining Questions}

\subsubsection{FA Oxidation in States of Reduced SCD1 Activity}

The protection from diet-induced obesity in Scd1-/- mice may stem from an increase in FA oxidation in addition to a reduction in lipogenesis [101]. Although the studies presented in this thesis did not directly examine FA oxidation, the microarray dataset from Chapter 3 demonstrated that SCD1 inhibition in adipocytes significantly up-regulated the expression of Cptl $\alpha$ but down-regulated the expression of acylcarnitine translocase (Cact) and Ppara. In addition, the expression of several other genes involved in FA oxidation and energy expenditure including Cpt2, Acadvl, Fat/Cd36, Ucpl-3, as well as Ampk isoforms, was unaltered in SCD1inhibited adipocytes. These results partly align with reports in the liver that Cpt1 expression is increased in Scd1-/- mice [15], and have now extended these observations to adipocytes with compromised SCD1 activity. Also in agreement with these results is work showing that BAT from Scd1-/- mice had increased rates of FA oxidation and Cpt1 expression compared to wild type mice [100, 113]. Interestingly, while the expression of genes including Ampk and Acadvl was increased in the liver of $S c d 1-/-$ mice $[15,100]$, these thesis results suggest that changes in FA oxidation markers within SCD1-inhibited adipocytes may not mirror the results in the liver of Scd1-/- mice. In adipocytes, Kim et al. noted that the expression of Ucp2 was not altered in SCD1-inhibited 3T3-L1 cells compared to control cells [163], which agrees with the microarray results from this thesis. However, Kim et al. did not observe significant changes in Cptl or Ppara expression, which conflicts with the microarray dataset of this thesis. Together, these results encourage the continued investigation of how compromised SCD1 activity influences FA 
oxidation and mitochondrial respiration in adipocytes by directly assessing FA oxidation as well as elements including CPT1 and CPT2 activity.

\subsubsection{Consideration of SCD2}

Although $S c d 1$ is the predominant isoform in AT, $S c d 2$ is also expressed in murine AT and adipocytes [10,35]; however, very few investigations have examined $S c d 2$ in adipocytes within the context of FA metabolism. This is intriguing given that $S c d 2$ catalyzes the same enzymatic reaction as $S c d 1$ [10], and thus could be expected to have similar effects in adipocytes as $S c d 1$. The work in this thesis has demonstrated that a reduction in adipocyte SCD1 activity did not significantly alter $S c d 2$ gene expression (aside from a very small increase at day 4 of differentiation). Although speculative, this may somewhat suggest that 3T3-L1 adipocytes conserve $S c d 2$ expression despite changes in SCD1 activity. In line with this, $S c d 2$ has been purported to have an important role in adipocyte differentiation [109]. Specifically, Christianson et al. knocked down $S c d 2$ expression in 3T3-L1 adipocytes using siRNA, and showed that $S c d 2$ was necessary for the induction of adipocyte-specific genes (e.g., Ppar $\gamma$ ) as well as the maintenance of adipogenesis and the adipocyte phenotype [109]. The work in this thesis did not measure SCD2 protein content, as there is not yet a high quality primary antibody for SCD2 that is commercially available. Additionally, a SCD2-specific inhibitor does not yet exist. In the future, should a high quality SCD2 antibody and an SCD2-specific inhibitor become available, it would be interesting to assess the differential impacts of SCD1 vs. SCD2 inhibition on adipocyte lipid metabolism. This would allow the specific examination of SCD1 and SCD2 enzyme activity, without knocking out the expression of the genes (e.g., using siRNA). 


\subsubsection{Prospective Research Question - Why is SCD1 Activity Correlated with Obesity?}

This thesis has provided significant evidence that reduced SCD1 activity can contribute to numerous detrimental effects in adipocytes. This could lead to the expectation that reduced SCD1 activity might be associated with health complications including obesity, yet this is not the case at the whole body level. Indeed, SCD1 activity in human adults (measured using plasma FA ratios) is often positively associated with obesity, the metabolic syndrome, plasma TAG levels, and insulin insensitivity $[130,131,133,134,149-151]$. However, it is very difficult to know whether elevated SCD1 activity is a contributing cause to obesity and obesity-related complications, or if it is a protective reaction to nutrient overload. Given that diets high in carbohydrates or SFAs will generally induce hepatic $S c d 1$ expression in mice, one might suppose that the induction of $S c d 1$ is an adaptive response to caloric overload $[10,16,60,74]$. Results in this thesis align with this idea, since SCD1 activity in adipocytes appeared to facilitate the management of exogenous FAs without the induction of inflammatory markers. Although speculative, perhaps the body attempts to compensate for caloric overload by up-regulating $S C D 1$ in an effort to channel calories into TAG-MUFAs for safe storage in adipocytes. Furthermore, the high SFA and sugar content of the Western diet is a perfect recipe for the induction of human $S C D 1$, which may be the main contributing factor to the positive correlation between SCD1 activity and obesity. It would therefore be extremely interesting to directly compare SCD1 activity in obese adults with different dietary habits (e.g. adults traditionally consuming a Western diet vs. a Mediterranean diet). 


\subsection{Concluding Remarks}

In summary, this thesis has improved our understanding of adipocyte SCD1 and has highlighted numerous important roles of SCD1 in adipocyte metabolism. Prior to this thesis, the majority of SCD1 research revolved around Scd1-/- mice and hepatic FA metabolism. This prior work has served as an excellent foundation for SCD1 research; however, our understanding of SCD1 in AT (a critical metabolic tissue linked with obesity and high SCD1 expression in humans and rodents) remained poorly understood. This was significant, since work in Scd1-/- mice had prompted the discussion that targeting SCD1 activity may be a viable therapeutic approach to manage obesity [169]. While studies using $S c d 1$-/- mice showed that reduced SCD1 activity caused lower total TAG levels in the liver, eyes and AT, the results in this thesis have further extended these findings in an adipocyte model and have supplemented this data with a proposed contributing mechanism. The reduction in TAG production in SCD1-inhibited adipocytes may have led to complications including an inability to properly store excess FAs. In turn, this may have contributed to the inability of SCD1-inhibited adipocytes to tolerate exogenous SFAs without the induction of inflammatory markers. Importantly, this thesis has also highlighted several important influences of SCD1 activity in adipocytes, including the prevention of DAGSFA accumulation and impairments in insulin signaling. Additionally, SCD1 activity may regulate the PL SFA:MUFA ratio, which could also influence cell metabolism and contribute to modifications in biological pathways (e.g., insulin signaling and cellular stress pathways). Overall, this thesis has presented several effects of inhibiting SCD1 activity that may have somewhat detrimental influences in adipocytes, and therefore provides evidence that reduced SCD1 activity may not be a suitable therapeutic target for obesity interventions. The prospective 
research avenues identified in this thesis will continue to improve our understanding of the wideranging impacts of SCD1 in adipocytes and overall human health. 


\section{$\underline{\text { References }}$}

[1] World Health Organization, Obesity and overweight, in, 2015.

[2] R.S. Ahima, M.A. Lazar, Physiology. The health risk of obesity--better metrics imperative, Science, 341 (2013) 856-858.

[3] K. Karastergiou, V. Mohamed-Ali, The autocrine and paracrine roles of adipokines, Mol Cell Endocrinol, 318 (2010) 69-78.

[4] S. Winer, Y. Chan, G. Paltser, D. Truong, H. Tsui, J. Bahrami, R. Dorfman, Y. Wang, J. Zielenski, F. Mastronardi, Y. Maezawa, D.J. Drucker, E. Engleman, D. Winer, H.M. Dosch, Normalization of obesity-associated insulin resistance through immunotherapy, Nat Med, 15 (2009) 921-929.

[5] A.T. Ali, W.E. Hochfeld, R. Myburgh, M.S. Pepper, Adipocyte and adipogenesis, Eur J Cell Biol, 92 (2013) 229-236.

[6] K. Sun, C.M. Kusminski, P.E. Scherer, Adipose tissue remodeling and obesity, J Clin Invest, 121 (2011) 2094-2101.

[7] R.M. Cowherd, R.E. Lyle, R.E. McGehee, Jr., Molecular regulation of adipocyte differentiation, Semin Cell Dev Biol, 10 (1999) 3-10.

[8] E. Warensjo, M. Rosell, M.L. Hellenius, B. Vessby, U. De Faire, U. Riserus, Associations between estimated fatty acid desaturase activities in serum lipids and adipose tissue in humans: links to obesity and insulin resistance, Lipids Health Dis, 8 (2009) 37.

[9] L. Hodson, B.A. Fielding, Stearoyl-CoA desaturase: rogue or innocent bystander?, Progress in Lipid Research, 52 (2013) 15-42.

[10] C.M. Paton, J.M. Ntambi, Biochemical and physiological function of stearoyl-CoA desaturase, Am J Physiol Endocrinol Metab, 297 (2009) E28-37.

[11] M. Miyazaki, Y.C. Kim, M.P. Gray-Keller, A.D. Attie, J.M. Ntambi, The biosynthesis of hepatic cholesterol esters and triglycerides is impaired in mice with a disruption of the gene for stearoyl-CoA desaturase 1, J Biol Chem, 275 (2000) 30132-30138.

[12] B. Garbay, F. Boiron-Sargueil, M. Shy, T. Chbihi, H. Jiang, J. Kamholz, C. Cassagne, Regulation of oleoyl-CoA synthesis in the peripheral nervous system: demonstration of a link with myelin synthesis, J Neurochem, 71 (1998) 1719-1726.

[13] H. Cao, K. Gerhold, J.R. Mayers, M.M. Wiest, S.M. Watkins, G.S. Hotamisligil, Identification of a lipokine, a lipid hormone linking adipose tissue to systemic metabolism, Cell, 134 (2008) 933-944.

[14] T. Shinomura, Y. Asaoka, M. Oka, K. Yoshida, Y. Nishizuka, Synergistic action of diacylglycerol and unsaturated fatty acid for protein kinase $\mathrm{C}$ activation: its possible implications, Proceedings of the National Academy of Sciences of the United States of America, 88 (1991) 5149-5153.

[15] J.M. Ntambi, M. Miyazaki, J.P. Stoehr, H. Lan, C.M. Kendziorski, B.S. Yandell, Y. Song, P. Cohen, J.M. Friedman, A.D. Attie, Loss of stearoyl-CoA desaturase-1 function protects mice against adiposity, Proceedings of the National Academy of Sciences, 99 (2002) 11482-11486.

[16] M. Miyazaki, Y.C. Kim, J.M. Ntambi, A lipogenic diet in mice with a disruption of the stearoyl-CoA desaturase 1 gene reveals a stringent requirement of endogenous monounsaturated fatty acids for triglyceride synthesis, J Lipid Res, 42 (2001) 1018-1024.

[17] M.L. MacDonald, M. van Eck, R.B. Hildebrand, B.W. Wong, N. Bissada, P. Ruddle, A. Kontush, H. Hussein, M.A. Pouladi, M.J. Chapman, C. Fievet, T.J. van Berkel, B. Staels, B.M. McManus, M.R. Hayden, Despite antiatherogenic metabolic characteristics, SCD1-deficient 
mice have increased inflammation and atherosclerosis, Arterioscler Thromb Vasc Biol, 29 (2009) 341-347.

[18] H. Sampath, J.M. Ntambi, The role of stearoyl-CoA desaturase in obesity, insulin resistance, and inflammation, Ann N Y Acad Sci, 1243 (2011) 47-53.

[19] J.M. Brown, S. Chung, J.K. Sawyer, C. Degirolamo, H.M. Alger, T. Nguyen, X. Zhu, M.N. Duong, A.L. Wibley, R. Shah, M.A. Davis, K. Kelley, M.D. Wilson, C. Kent, J.S. Parks, L.L. Rudel, Inhibition of stearoyl-coenzyme A desaturase 1 dissociates insulin resistance and obesity from atherosclerosis, Circulation, 118 (2008) 1467-1475.

[20] K. Thorn, M. Hovsepyan, P. Bergsten, Reduced levels of SCD1 accentuate palmitateinduced stress in insulin-producing beta-cells, Lipids Health Dis, 9 (2010) 108.

[21] M. Miyazaki, M.J. Jacobson, W.C. Man, P. Cohen, E. Asilmaz, J.M. Friedman, J.M. Ntambi, Identification and characterization of murine SCD4, a novel heart-specific stearoyl-CoA desaturase isoform regulated by leptin and dietary factors, J Biol Chem, 278 (2003) 3390433911.

[22] Y. Zheng, S.M. Prouty, A. Harmon, J.P. Sundberg, K.S. Stenn, S. Parimoo, Scd3--a novel gene of the stearoyl-CoA desaturase family with restricted expression in skin, Genomics, 71 (2001) 182-191.

[23] M. Miyazaki, M.T. Flowers, H. Sampath, K. Chu, C. Otzelberger, X. Liu, J.M. Ntambi, Hepatic stearoyl-CoA desaturase-1 deficiency protects mice from carbohydrate-induced adiposity and hepatic steatosis, Cell Metab, 6 (2007) 484-496.

[24] A. Peter, A. Cegan, S. Wagner, R. Lehmann, N. Stefan, A. Konigsrainer, I. Konigsrainer, H.U. Haring, E. Schleicher, Hepatic lipid composition and stearoyl-coenzyme A desaturase 1 mRNA expression can be estimated from plasma VLDL fatty acid ratios, Clin Chem, 55 (2009) 2113-2120.

[25] E. Asilmaz, P. Cohen, M. Miyazaki, P. Dobrzyn, K. Ueki, G. Fayzikhodjaeva, A.A. Soukas, C.R. Kahn, J.M. Ntambi, N.D. Socci, J.M. Friedman, Site and mechanism of leptin action in a rodent form of congenital lipodystrophy, J Clin Invest, 113 (2004) 414-424.

[26] M. Miyazaki, A. Dobrzyn, W.C. Man, K. Chu, H. Sampath, H.J. Kim, J.M. Ntambi, Stearoyl-CoA desaturase 1 gene expression is necessary for fructose-mediated induction of lipogenic gene expression by sterol regulatory element-binding protein-1c-dependent and independent mechanisms, J Biol Chem, 279 (2004) 25164-25171.

[27] C.K. Hyun, E.D. Kim, M.T. Flowers, X. Liu, E. Kim, M. Strable, J.M. Ntambi, Adiposespecific deletion of stearoyl-CoA desaturase 1 up-regulates the glucose transporter GLUT1 in adipose tissue, Biochem Biophys Res Commun, 399 (2010) 480-486.

[28] M.T. Flowers, J.M. Ntambi, Role of stearoyl-coenzyme A desaturase in regulating lipid metabolism, Curr Opin Lipidol, 19 (2008) 248-256.

[29] M. Miyazaki, W.C. Man, J.M. Ntambi, Targeted disruption of stearoyl-CoA desaturase1 gene in mice causes atrophy of sebaceous and meibomian glands and depletion of wax esters in the eyelid, J Nutr, 131 (2001) 2260-2268.

[30] M. Miyazaki, H.J. Kim, W.C. Man, J.M. Ntambi, Oleoyl-CoA is the major de novo product of stearoyl-CoA desaturase 1 gene isoform and substrate for the biosynthesis of the Harderian gland 1-alkyl-2,3-diacylglycerol, J Biol Chem, 276 (2001) 39455-39461.

[31] M. Miyazaki, F.E. Gomez, J.M. Ntambi, Lack of stearoyl-CoA desaturase-1 function induces a palmitoyl-CoA Delta6 desaturase and represses the stearoyl-CoA desaturase-3 gene in the preputial glands of the mouse, J Lipid Res, 43 (2002) 2146-2154. 
[32] M. Miyazaki, J.M. Ntambi, Role of stearoyl-coenzyme A desaturase in lipid metabolism, Prostaglandins Leukot Essent Fatty Acids, 68 (2003) 113-121.

[33] F.S. Heinemann, J. Ozols, Stearoyl-CoA desaturase, a short-lived protein of endoplasmic reticulum with multiple control mechanisms, Prostaglandins Leukot Essent Fatty Acids, 68 (2003) 123-133.

[34] J.M. Ntambi, S.A. Buhrow, K.H. Kaestner, R.J. Christy, E. Sibley, T.J. Kelly, Jr., M.D. Lane, Differentiation-induced gene expression in 3T3-L1 preadipocytes. Characterization of a differentially expressed gene encoding stearoyl-CoA desaturase, J Biol Chem, 263 (1988) 17291-17300.

[35] K.H. Kaestner, J.M. Ntambi, T.J. Kelly, Jr., M.D. Lane, Differentiation-induced gene expression in 3T3-L1 preadipocytes. A second differentially expressed gene encoding stearoylCoA desaturase, J Biol Chem, 264 (1989) 14755-14761.

[36] M. Miyazaki, A. Dobrzyn, P.M. Elias, J.M. Ntambi, Stearoyl-CoA desaturase-2 gene expression is required for lipid synthesis during early skin and liver development, Proc Natl Acad Sci U S A, 102 (2005) 12501-12506.

[37] J. Wang, L. Yu, R.E. Schmidt, C. Su, X. Huang, K. Gould, G. Cao, Characterization of HSCD5, a novel human stearoyl-CoA desaturase unique to primates, Biochem Biophys Res Commun, 332 (2005) 735-742.

[38] L. Zhang, L. Ge, T. Tran, K. Stenn, S.M. Prouty, Isolation and characterization of the human stearoyl-CoA desaturase gene promoter: requirement of a conserved CCAAT ciselement, Biochem J, 357 (2001) 183-193.

[39] H. Sampath, J.M. Ntambi, Role of stearoyl-CoA desaturase-1 in skin integrity and whole body energy balance, J Biol Chem, 289 (2014) 2482-2488.

[40] L. Zhang, L. Ge, S. Parimoo, K. Stenn, S.M. Prouty, Human stearoyl-CoA desaturase: alternative transcripts generated from a single gene by usage of tandem polyadenylation sites, Biochem J, 340 ( Pt 1) (1999) 255-264.

[41] M.W. Hulver, J.R. Berggren, M.J. Carper, M. Miyazaki, J.M. Ntambi, E.P. Hoffman, J.P. Thyfault, R. Stevens, G.L. Dohm, J.A. Houmard, D.M. Muoio, Elevated stearoyl-CoA desaturase-1 expression in skeletal muscle contributes to abnormal fatty acid partitioning in obese humans, Cell Metab, 2 (2005) 251-261.

[42] S. Zhang, Y. Yang, Y. Shi, Characterization of human SCD2, an oligomeric desaturase with improved stability and enzyme activity by cross-linking in intact cells, Biochem J, 388 (2005) 135-142.

[43] S. Beiraghi, M. Zhou, C.B. Talmadge, N. Went-Sumegi, J.R. Davis, D. Huang, H. Saal, T.A. Seemayer, J. Sumegi, Identification and characterization of a novel gene disrupted by a pericentric inversion inv(4)(p13.1q21.1) in a family with cleft lip, Gene, 309 (2003) 11-21.

[44] H. Bene, D. Lasky, J.M. Ntambi, Cloning and characterization of the human stearoyl-CoA desaturase gene promoter: transcriptional activation by sterol regulatory element binding protein and repression by polyunsaturated fatty acids and cholesterol, Biochem Biophys Res Commun, 284 (2001) 1194-1198.

[45] K. Mihara, Structure and regulation of rat liver microsomal stearoyl-CoA desaturase gene, J Biochem, 108 (1990) 1022-1029.

[46] M.A. Thiede, J. Ozols, P. Strittmatter, Construction and sequence of cDNA for rat liver stearyl coenzyme A desaturase, J Biol Chem, 261 (1986) 13230-13235. 
[47] R.J. Ward, M.T. Travers, S.E. Richards, R.G. Vernon, A.M. Salter, P.J. Buttery, M.C. Barber, Stearoyl-CoA desaturase mRNA is transcribed from a single gene in the ovine genome, Biochim Biophys Acta, 1391 (1998) 145-156.

[48] D.E. Tabor, J.B. Kim, B.M. Spiegelman, P.A. Edwards, Transcriptional activation of the stearoyl-CoA desaturase 2 gene by sterol regulatory element-binding protein/adipocyte determination and differentiation factor 1, J Biol Chem, 273 (1998) 22052-22058.

[49] J.E. Stukey, V.M. McDonough, C.E. Martin, The OLE1 gene of Saccharomyces cerevisiae encodes the delta 9 fatty acid desaturase and can be functionally replaced by the rat stearoyl-CoA desaturase gene, J Biol Chem, 265 (1990) 20144-20149.

[50] R. Dallerac, C. Labeur, J.-M. Jallon, D.C. Knipple, W.L. Roelofs, C. Wicker-Thomas, A $\Delta 9$ desaturase gene with a different substrate specificity is responsible for the cuticular diene hydrocarbon polymorphism in Drosophila melanogaster, Proceedings of the National Academy of Sciences of the United States of America, 97 (2000) 9449-9454.

[51] P. Wongwathanarat, L.V. Michaelson, A.T. Carter, C.M. Lazarus, G. Griffiths, A.K. Stobart, D.B. Archer, D.A. MacKenzie, Two fatty acid delta9-desaturase genes, ole1 and ole2, from Mortierella alpina complement the yeast ole1 mutation, Microbiology, 145 ( Pt 10) (1999) 2939-2946.

[52] J.L. Watts, J. Browse, A palmitoyl-CoA-specific delta9 fatty acid desaturase from Caenorhabditis elegans, Biochem Biophys Res Commun, 272 (2000) 263-269.

[53] Y.C. Kim, F.E. Gomez, B.G. Fox, J.M. Ntambi, Differential regulation of the stearoyl-CoA desaturase genes by thiazolidinediones in 3T3-L1 adipocytes, J Lipid Res, 41 (2000) 1310-1316.

[54] H.G. Enoch, A. Catala, P. Strittmatter, Mechanism of rat liver microsomal stearyl-CoA desaturase. Studies of the substrate specificity, enzyme-substrate interactions, and the function of lipid, J Biol Chem, 251 (1976) 5095-5103.

[55] M. Miyazaki, S.M. Bruggink, J.M. Ntambi, Identification of mouse palmitoyl-coenzyme A Delta9-desaturase, J Lipid Res, 47 (2006) 700-704.

[56] J.K. Yee, C.S. Mao, H.S. Hummel, S. Lim, S. Sugano, V.K. Rehan, G. Xiao, W.N. Lee, Compartmentalization of stearoyl-coenzyme A desaturase 1 activity in HepG2 cells, J Lipid Res, 49 (2008) 2124-2134.

[57] S.K. Rhee, A.J. Kayani, A. Ciszek, J.T. Brenna, Desaturation and interconversion of dietary stearic and palmitic acids in human plasma and lipoproteins, Am J Clin Nutr, 65 (1997) 451-458. [58] D. Mauvoisin, C. Mounier, Hormonal and nutritional regulation of SCD1 gene expression, Biochimie, 93 (2011) 78-86.

[59] R.J. Christy, V.W. Yang, J.M. Ntambi, D.E. Geiman, W.H. Landschulz, A.D. Friedman, Y. Nakabeppu, T.J. Kelly, M.D. Lane, Differentiation-induced gene expression in 3T3-L1 preadipocytes: CCAAT/enhancer binding protein interacts with and activates the promoters of two adipocyte-specific genes, Genes Dev, 3 (1989) 1323-1335.

[60] M.F. Chong, L. Hodson, A.S. Bickerton, R. Roberts, M. Neville, F. Karpe, K.N. Frayn, B.A. Fielding, Parallel activation of de novo lipogenesis and stearoyl-CoA desaturase activity after $3 \mathrm{~d}$ of high-carbohydrate feeding, Am J Clin Nutr, 87 (2008) 817-823.

[61] S.B. Biddinger, K. Almind, M. Miyazaki, E. Kokkotou, J.M. Ntambi, C.R. Kahn, Effects of diet and genetic background on sterol regulatory element-binding protein-1c, stearoyl-CoA desaturase 1, and the development of the metabolic syndrome, Diabetes, 54 (2005) 1314-1323.

[62] H. Sampath, M. Miyazaki, A. Dobrzyn, J.M. Ntambi, Stearoyl-CoA desaturase-1 mediates the pro-lipogenic effects of dietary saturated fat, J Biol Chem, 282 (2007) 2483-2493. 
[63] J. Lin, R. Yang, P.T. Tarr, P.H. Wu, C. Handschin, S. Li, W. Yang, L. Pei, M. Uldry, P. Tontonoz, C.B. Newgard, B.M. Spiegelman, Hyperlipidemic effects of dietary saturated fats mediated through PGC-1beta coactivation of SREBP, Cell, 120 (2005) 261-273.

[64] B. Shaw, S. Lambert, M.H. Wong, J.C. Ralston, C. Stryjecki, D.M. Mutch, Individual saturated and monounsaturated fatty acids trigger distinct transcriptional networks in differentiated 3T3-L1 preadipocytes, J Nutrigenet Nutrigenomics, 6 (2013) 1-15.

[65] U. Riserus, G.D. Tan, B.A. Fielding, M.J. Neville, J. Currie, D.B. Savage, V.K. Chatterjee, K.N. Frayn, S. O'Rahilly, F. Karpe, Rosiglitazone increases indexes of stearoyl-CoA desaturase activity in humans: link to insulin sensitization and the role of dominant-negative mutation in peroxisome proliferator-activated receptor-gamma, Diabetes, 54 (2005) 1379-1384.

[66] Y.C. Kim, J.M. Ntambi, Regulation of stearoyl-CoA desaturase genes: role in cellular metabolism and preadipocyte differentiation, Biochem Biophys Res Commun, 266 (1999) 1-4.

[67] S. Kurebayashi, T. Hirose, Y. Miyashita, S. Kasayama, T. Kishimoto, Thiazolidinediones downregulate stearoyl-CoA desaturase 1 gene expression in 3T3-L1 adipocytes, Diabetes, 46 (1997) 2115-2118.

[68] M. Gao, L. Bu, Y. Ma, D. Liu, Concurrent activation of liver X receptor and peroxisome proliferator-activated receptor alpha exacerbates hepatic steatosis in high fat diet-induced obese mice, PLoS ONE, 8 (2013) e65641.

[69] A. Peter, C. Weigert, H. Staiger, F. Machicao, F. Schick, J. Machann, N. Stefan, C. Thamer, H.U. Haring, E. Schleicher, Individual stearoyl-coa desaturase 1 expression modulates endoplasmic reticulum stress and inflammation in human myotubes and is associated with skeletal muscle lipid storage and insulin sensitivity in vivo, Diabetes, 58 (2009) 1757-1765.

[70] D.B. Jump, S.D. Clarke, Regulation of gene expression by dietary fat, Annu Rev Nutr, 19 (1999) 63-90.

[71] A.M. Sessler, N. Kaur, J.P. Palta, J.M. Ntambi, Regulation of Stearoyl-CoA Desaturase 1 mRNA Stability by Polyunsaturated Fatty Acids in 3T3-L1 Adipocytes, Journal of Biological Chemistry, 271 (1996) 29854-29858.

[72] H. Sampath, J.M. Ntambi, Polyunsaturated fatty acid regulation of genes of lipid metabolism, Annu Rev Nutr, 25 (2005) 317-340.

[73] H.-J. Kim, M. Miyazaki, J.M. Ntambi, Dietary cholesterol opposes PUFA-mediated repression of the stearoyl-CoA desaturase-1 gene by SREBP-1 independent mechanism, Journal of Lipid Research, 43 (2002) 1750-1757.

[74] J.M. Ntambi, Dietary regulation of stearoyl-CoA desaturase 1 gene expression in mouse liver, J Biol Chem, 267 (1992) 10925-10930.

[75] K. Iizuka, R.K. Bruick, G. Liang, J.D. Horton, K. Uyeda, Deficiency of carbohydrate response element-binding protein (ChREBP) reduces lipogenesis as well as glycolysis, Proc Natl Acad Sci U S A, 101 (2004) 7281-7286.

[76] D. Mauvoisin, G. Rocque, O. Arfa, A. Radenne, P. Boissier, C. Mounier, Role of the PI3kinase/mTor pathway in the regulation of the stearoyl CoA desaturase (SCD1) gene expression by insulin in liver, J Cell Commun Signal, 1 (2007) 113-125.

[77] R. Jeffcoat, P.A. Roberts, J. Ormesher, A.T. James, Stearolyl-CoA desaturase: a control enzyme in hepatic lipogenesis, Eur J Biochem, 101 (1979) 439-445.

[78] K.M. Waters, J.M. Ntambi, Insulin and dietary fructose induce stearoyl-CoA desaturase 1 gene expression of diabetic mice, J Biol Chem, 269 (1994) 27773-27777. 
[79] E. Kim, N.C. Liu, I.C. Yu, H.Y. Lin, Y.F. Lee, J.D. Sparks, L.M. Chen, C. Chang, Metformin inhibits nuclear receptor TR4-mediated hepatic stearoyl-CoA desaturase 1 gene expression with altered insulin sensitivity, Diabetes, 60 (2011) 1493-1503.

[80] S. Le Lay, I. Lefrere, C. Trautwein, I. Dugail, S. Krief, Insulin and sterol-regulatory element-binding protein-1c (SREBP-1C) regulation of gene expression in 3T3-L1 adipocytes. Identification of CCAAT/enhancer-binding protein beta as an SREBP-1C target, J Biol Chem, 277 (2002) 35625-35634.

[81] D.E. Tabor, J.B. Kim, B.M. Spiegelman, P.A. Edwards, Identification of conserved ciselements and transcription factors required for sterol-regulated transcription of stearoyl-CoA desaturase 1 and 2, J Biol Chem, 274 (1999) 20603-20610.

[82] M.V. Singh, J.M. Ntambi, Nuclear factor 1 is essential for the expression of stearoyl-CoA desaturase 1 gene during preadipocyte differentiation, Biochim Biophys Acta, 1398 (1998) 148156.

[83] J.M. Ntambi, M. Miyazaki, A. Dobrzyn, Regulation of stearoyl-CoA desaturase expression, Lipids, 39 (2004) 1061-1065.

[84] W. Zhang, M.A. Della-Fera, D.L. Hartzell, D. Hausman, C.A. Baile, Adipose tissue gene expression profiles in ob/ob mice treated with leptin, Life Sci, 83 (2008) 35-42.

[85] S.B. Biddinger, M. Miyazaki, J. Boucher, J.M. Ntambi, C.R. Kahn, Leptin suppresses stearoyl-CoA desaturase 1 by mechanisms independent of insulin and sterol regulatory elementbinding protein-1c, Diabetes, 55 (2006) 2032-2041.

[86] D. Mauvoisin, M. Prevost, S. Ducheix, M.P. Arnaud, C. Mounier, Key role of the ERK1/2 MAPK pathway in the transcriptional regulation of the Stearoyl-CoA Desaturase (SCD1) gene expression in response to leptin, Mol Cell Endocrinol, 319 (2010) 116-128.

[87] L. Lundholm, H. Zang, A.L. Hirschberg, J.A. Gustafsson, P. Arner, K. Dahlman-Wright, Key lipogenic gene expression can be decreased by estrogen in human adipose tissue, Fertil Steril, 90 (2008) 44-48.

[88] G. Bryzgalova, L. Lundholm, N. Portwood, J.A. Gustafsson, A. Khan, S. Efendic, K. Dahlman-Wright, Mechanisms of antidiabetogenic and body weight-lowering effects of estrogen in high-fat diet-fed mice, Am J Physiol Endocrinol Metab, 295 (2008) E904-912.

[89] G. Bryzgalova, H. Gao, B. Ahren, J.R. Zierath, D. Galuska, T.L. Steiler, K. DahlmanWright, S. Nilsson, J.A. Gustafsson, S. Efendic, A. Khan, Evidence that oestrogen receptor-alpha plays an important role in the regulation of glucose homeostasis in mice: insulin sensitivity in the liver, Diabetologia, 49 (2006) 588-597.

[90] H. Gao, G. Bryzgalova, E. Hedman, A. Khan, S. Efendic, J.A. Gustafsson, K. DahlmanWright, Long-term administration of estradiol decreases expression of hepatic lipogenic genes and improves insulin sensitivity in ob/ob mice: a possible mechanism is through direct regulation of signal transducer and activator of transcription 3, Mol Endocrinol, 20 (2006) 1287-1299.

[91] K.M. Waters, C.W. Miller, J.M. Ntambi, Localization of a negative thyroid hormoneresponse region in hepatic stearoyl-CoA desaturase gene 1, Biochem Biophys Res Commun, 233 (1997) 838-843.

[92] R.L. Stone, D.A. Bernlohr, The molecular basis for inhibition of adipose conversion of murine 3T3-L1 cells by retinoic acid, Differentiation, 45 (1990) 119-127.

[93] C.W. Miller, K.M. Waters, J.M. Ntambi, Regulation of hepatic stearoyl-CoA desaturase gene 1 by vitamin A, Biochem Biophys Res Commun, 231 (1997) 206-210. 
[94] J.M. Landau, A. Sekowski, M.W. Hamm, Dietary cholesterol and the activity of stearoyl CoA desaturase in rats: evidence for an indirect regulatory effect, Biochim Biophys Acta, 1345 (1997) 349-357.

[95] M.L. Garg, A.A. Wierzbicki, A.B. Thomson, M.T. Clandinin, Dietary cholesterol and/or n-3 fatty acid modulate delta 9-desaturase activity in rat liver microsomes, Biochim Biophys Acta, 962 (1988) 330-336.

[96] P. Georgel, K. Crozat, X. Lauth, E. Makrantonaki, H. Seltmann, S. Sovath, K. Hoebe, X. Du, S. Rutschmann, Z. Jiang, T. Bigby, V. Nizet, C.C. Zouboulis, B. Beutler, A toll-like receptor 2-responsive lipid effector pathway protects mammals against skin infections with gram-positive bacteria, Infect Immun, 73 (2005) 4512-4521.

[97] T. Toyama, N. Kudo, A. Mitsumoto, Y. Hibino, T. Tsuda, Y. Kawashima, Stearoyl-CoA desaturase activity is elevated by the suppression of its degradation by clofibric acid in the liver of rats, J Pharmacol Sci, 103 (2007) 383-390.

[98] H. Kato, K. Sakaki, K. Mihara, Ubiquitin-proteasome-dependent degradation of mammalian ER stearoyl-CoA desaturase, J Cell Sci, 119 (2006) 2342-2353.

[99] S. Garcia-Serrano, I. Moreno-Santos, L. Garrido-Sanchez, C. Gutierrez-Repiso, J.M. Garcia-Almeida, J. Garcia-Arnes, J. Rivas-Marin, J.L. Gallego-Perales, E. Garcia-Escobar, G. Rojo-Martinez, F. Tinahones, F. Soriguer, M. Macias-Gonzalez, E. Garcia-Fuentes, StearoylCoA desaturase-1 is associated with insulin resistance in morbidly obese subjects, Mol Med, 17 (2011) 273-280.

[100] P. Dobrzyn, A. Dobrzyn, M. Miyazaki, P. Cohen, E. Asilmaz, D.G. Hardie, J.M. Friedman, J.M. Ntambi, Stearoyl-CoA desaturase 1 deficiency increases fatty acid oxidation by activating AMP-activated protein kinase in liver, Proc Natl Acad Sci U S A, 101 (2004) 6409-6414.

[101] W.C. Man, M. Miyazaki, K. Chu, J. Ntambi, Colocalization of SCD1 and DGAT2: implying preference for endogenous monounsaturated fatty acids in triglyceride synthesis, J Lipid Res, 47 (2006) 1928-1939.

[102] Y. Zheng, K.J. Eilertsen, L. Ge, L. Zhang, J.P. Sundberg, S.M. Prouty, K.S. Stenn, S. Parimoo, Scd1 is expressed in sebaceous glands and is disrupted in the asebia mouse, Nat Genet, 23 (1999) 268-270.

[103] C.A. Harris, J.T. Haas, R.S. Streeper, S.J. Stone, M. Kumari, K. Yang, X. Han, N. Brownell, R.W. Gross, R. Zechner, R.V. Farese, Jr., DGAT enzymes are required for triacylglycerol synthesis and lipid droplets in adipocytes, J Lipid Res, 52 (2011) 657-667.

[104] V.A. Zammit, Hepatic triacylglycerol synthesis and secretion: DGAT2 as the link between glycaemia and triglyceridaemia, Biochem J, 451 (2013) 1-12.

[105] S.B. Weiss, E.P. Kennedy, J.Y. Kiyasu, The enzymatic synthesis of triglycerides, J Biol Chem, 235 (1960) 40-44.

[106] A. Nilsson, R. Sundler, B. Akesson, Effect of different albumin-bound fatty acids on fatty acid and cholesterol biosynthesis in rat hepatocytes, FEBS Lett, 45 (1974) 282-285.

[107] S.P. Poulos, M.V. Dodson, G.J. Hausman, Cell line models for differentiation: preadipocytes and adipocytes, Exp Biol Med (Maywood), 235 (2010) 1185-1193.

[108] T.C. Otto, M.D. Lane, Adipose development: from stem cell to adipocyte, Crit Rev Biochem Mol Biol, 40 (2005) 229-242.

[109] J.L. Christianson, S. Nicoloro, J. Straubhaar, M.P. Czech, Stearoyl-CoA desaturase 2 is required for peroxisome proliferator-activated receptor gamma expression and adipogenesis in cultured 3T3-L1 cells, J Biol Chem, 283 (2008) 2906-2916. 
[110] R. Kasturi, V.C. Joshi, Hormonal regulation of stearoyl coenzyme A desaturase activity and lipogenesis during adipose conversion of 3T3-L1 cells, Journal of Biological Chemistry, 257 (1982) 12224-12230.

[111] F.T. Lin, M.D. Lane, Antisense CCAAT/enhancer-binding protein RNA suppresses coordinate gene expression and triglyceride accumulation during differentiation of 3T3-L1 preadipocytes, Genes Dev, 6 (1992) 533-544.

[112] J.K. Yee, P.N. Wahjudi, J. Vega, S. Lim, A. Martin, M.E. Patterson, J.N. Cohen, C.S. Mao, W.N. Lee, Stearoyl-CoA desaturase enzyme 1 inhibition reduces glucose utilization for de novo fatty acid synthesis and cell proliferation in 3T3-L1 adipocytes, Metabolomics, 9 (2013) 809-816.

[113] S.H. Lee, A. Dobrzyn, P. Dobrzyn, S.M. Rahman, M. Miyazaki, J.M. Ntambi, Lack of stearoyl-CoA desaturase 1 upregulates basal thermogenesis but causes hypothermia in a cold environment, J Lipid Res, 45 (2004) 1674-1682.

[114] F. Yap, L. Craddock, J. Yang, Mechanism of AMPK suppression of LXR-dependent Srebp-1c transcription, Int J Biol Sci, 7 (2011) 645-650.

[115] E. Binczek, B. Jenke, B. Holz, R.H. Gunter, M. Thevis, W. Stoffel, Obesity resistance of the stearoyl-CoA desaturase-deficient (scd1-/-) mouse results from disruption of the epidermal lipid barrier and adaptive thermoregulation, Biol Chem, 388 (2007) 405-418.

[116] M.T. Flowers, C.M. Paton, S.M. O'Byrne, K. Schiesser, J.A. Dawson, W.S. Blaner, C. Kendziorski, J.M. Ntambi, Metabolic changes in skin caused by Scd1 deficiency: a focus on retinol metabolism, PLoS ONE, 6 (2011) e19734.

[117] C. Chen, Y.M. Shah, K. Morimura, K.W. Krausz, M. Miyazaki, T.A. Richardson, E.T. Morgan, J.M. Ntambi, J.R. Idle, F.J. Gonzalez, Metabolomics reveals that hepatic stearoyl-CoA desaturase 1 downregulation exacerbates inflammation and acute colitis, Cell Metab, 7 (2008) 135-147.

[118] M. Caron-Jobin, D. Mauvoisin, A. Michaud, A. Veilleux, S. Noel, M.P. Fortier, P. Julien, A. Tchernof, C. Mounier, Stearic acid content of abdominal adipose tissues in obese women, Nutr Diabetes, 2 (2012) e23.

[119] A. Peter, C. Weigert, H. Staiger, K. Rittig, A. Cegan, P. Lutz, F. Machicao, H.U. Haring, E. Schleicher, Induction of stearoyl-CoA desaturase protects human arterial endothelial cells against lipotoxicity, Am J Physiol Endocrinol Metab, 295 (2008) E339-349.

[120] X. Liu, M. Miyazaki, M.T. Flowers, H. Sampath, M. Zhao, K. Chu, C.M. Paton, D.S. Joo, J.M. Ntambi, Loss of Stearoyl-CoA desaturase-1 attenuates adipocyte inflammation: effects of adipocyte-derived oleate, Arterioscler Thromb Vasc Biol, 30 (2010) 31-38.

[121] H. Petersson, S. Basu, T. Cederholm, U. Riserus, Serum fatty acid composition and indices of stearoyl-CoA desaturase activity are associated with systemic inflammation: longitudinal analyses in middle-aged men, Br J Nutr, 99 (2008) 1186-1189.

[122] H. Petersson, L. Lind, J. Hulthe, A. Elmgren, T. Cederholm, U. Riserus, Relationships between serum fatty acid composition and multiple markers of inflammation and endothelial function in an elderly population, Atherosclerosis, 203 (2009) 298-303.

[123] H. Ariyama, N. Kono, S. Matsuda, T. Inoue, H. Arai, Decrease in membrane phospholipid unsaturation induces unfolded protein response, J Biol Chem, 285 (2010) 22027-22035.

[124] A. Dobrzyn, P. Dobrzyn, M. Miyazaki, H. Sampath, K. Chu, J.M. Ntambi, Stearoyl-CoA Desaturase 1 Deficiency Increases CTP:Choline Cytidylyltransferase Translocation into the Membrane and Enhances Phosphatidylcholine Synthesis in Liver, Journal of Biological Chemistry, 280 (2005) 23356-23362. 
[125] J.M. Collins, M.J. Neville, M.B. Hoppa, K.N. Frayn, De novo lipogenesis and stearoylCoA desaturase are coordinately regulated in the human adipocyte and protect against palmitateinduced cell injury, J Biol Chem, 285 (2010) 6044-6052.

[126] P. Clarys, A. Barel, Quantitative evaluation of skin surface lipids, Clin Dermatol, 13 (1995) 307-321.

[127] B.D. Sullivan, J.E. Evans, K.L. Krenzer, M. Reza Dana, D.A. Sullivan, Impact of antiandrogen treatment on the fatty acid profile of neutral lipids in human meibomian gland secretions, J Clin Endocrinol Metab, 85 (2000) 4866-4873.

[128] G.F. Gibbons, Assembly and secretion of hepatic very-low-density lipoprotein, Biochem J, 268 (1990) 1-13.

[129] N. Stefan, A. Peter, A. Cegan, H. Staiger, J. Machann, F. Schick, C.D. Claussen, A. Fritsche, H.U. Haring, E. Schleicher, Low hepatic stearoyl-CoA desaturase 1 activity is associated with fatty liver and insulin resistance in obese humans, Diabetologia, 51 (2008) 648656.

[130] A.D. Attie, R.M. Krauss, M.P. Gray-Keller, A. Brownlie, M. Miyazaki, J.J. Kastelein, A.J. Lusis, A.F. Stalenhoef, J.P. Stoehr, M.R. Hayden, J.M. Ntambi, Relationship between stearoylCoA desaturase activity and plasma triglycerides in human and mouse hypertriglyceridemia, $\mathrm{J}$ Lipid Res, 43 (2002) 1899-1907.

[131] E. Warensjo, U. Riserus, B. Vessby, Fatty acid composition of serum lipids predicts the development of the metabolic syndrome in men, Diabetologia, 48 (2005) 1999-2005.

[132] H. Sampath, J.M. Ntambi, Role of stearoyl-CoA desaturase in human metabolic disease, Future Lipidology, 3 (2008) 163-173.

[133] E. Corpeleijn, E.J. Feskens, E.H. Jansen, M. Mensink, W.H. Saris, T.W. de Bruin, E.E. Blaak, Improvements in glucose tolerance and insulin sensitivity after lifestyle intervention are related to changes in serum fatty acid profile and desaturase activities: the SLIM study, Diabetologia, 49 (2006) 2392-2401.

[134] F. Paillard, D. Catheline, F.L. Duff, M. Bouriel, Y. Deugnier, M. Pouchard, J.C. Daubert, P. Legrand, Plasma palmitoleic acid, a product of stearoyl-coA desaturase activity, is an independent marker of triglyceridemia and abdominal adiposity, Nutr Metab Cardiovasc Dis, 18 (2008) 436-440.

[135] E. Warensjo, M. Ohrvall, B. Vessby, Fatty acid composition and estimated desaturase activities are associated with obesity and lifestyle variables in men and women, Nutr Metab Cardiovasc Dis, 16 (2006) 128-136.

[136] C. Stryjecki, K. Roke, S. Clarke, D. Nielsen, A. Badawi, A. El-Sohemy, D.W. Ma, D.M. Mutch, Enzymatic activity and genetic variation in SCD1 modulate the relationship between fatty acids and inflammation, Mol Genet Metab, (2011).

[137] E. Warensjo, E. Ingelsson, P. Lundmark, L. Lannfelt, A.C. Syvanen, B. Vessby, U. Riserus, Polymorphisms in the SCD1 gene: associations with body fat distribution and insulin sensitivity, Obesity (Silver Spring), 15 (2007) 1732-1740.

[138] J. Pearce, Fatty acid synthesis in liver and adipose tissue, Proc Nutr Soc, 42 (1983) 263271.

[139] A.I. Su, T. Wiltshire, S. Batalov, H. Lapp, K.A. Ching, D. Block, J. Zhang, R. Soden, M. Hayakawa, G. Kreiman, M.P. Cooke, J.R. Walker, J.B. Hogenesch, A gene atlas of the mouse and human protein-encoding transcriptomes, Proc Natl Acad Sci U S A, 101 (2004) 6062-6067.

[140] C. Stryjecki, D.M. Mutch, Fatty acid-gene interactions, adipokines and obesity, Eur J Clin Nutr, 65 (2011) 285-297. 
[141] S. Nair, Y.H. Lee, E. Rousseau, P.A. Tataranni, L.J. Baier, C. Bogardus, M. Cam, P.A. Permana, Increased expression of inflammation-related genes in cultured preadipocytes/stromal vascular cells from obese compared with non-obese Pima Indians, Diabetologia, 48 (2005) 17841788 .

[142] N. Halberg, I. Wernstedt-Asterholm, P.E. Scherer, The adipocyte as an endocrine cell, Endocrinol Metab Clin North Am, 37 (2008) 753-768, x-xi.

[143] S. Galic, J.S. Oakhill, G.R. Steinberg, Adipose tissue as an endocrine organ, Mol Cell Endocrinol, 316 (2010) 129-139.

[144] P. Trayhurn, I.S. Wood, Adipokines: inflammation and the pleiotropic role of white adipose tissue, Br J Nutr, 92 (2004) 347-355.

[145] B.H. Jones, M.A. Maher, W.J. Banz, M.B. Zemel, J. Whelan, P.J. Smith, N. Moustaid, Adipose tissue stearoyl-CoA desaturase mRNA is increased by obesity and decreased by polyunsaturated fatty acids, Am J Physiol, 271 (1996) E44-49.

[146] J. Turyn, M. Stojek, J. Swierczynski, Up-regulation of stearoyl-CoA desaturase 1 and elongase 6 genes expression in rat lipogenic tissues by chronic food restriction and chronic food restriction/refeeding, Mol Cell Biochem, 345 (2010) 181-188.

[147] D. Mainieri, S. Summermatter, J. Seydoux, J.P. Montani, S. Rusconi, A.P. Russell, O. Boss, A.J. Buchala, A.G. Dulloo, A role for skeletal muscle stearoyl-CoA desaturase 1 in control of thermogenesis, FASEB J, 20 (2006) 1751-1753.

[148] L.M. Mangravite, K. Dawson, R.R. Davis, J.P. Gregg, R.M. Krauss, Fatty acid desaturase regulation in adipose tissue by dietary composition is independent of weight loss and is correlated with the plasma triacylglycerol response, Am J Clin Nutr, 86 (2007) 759-767.

[149] P. Sjogren, J. Sierra-Johnson, K. Gertow, M. Rosell, B. Vessby, U. de Faire, A. Hamsten, M.L. Hellenius, R.M. Fisher, Fatty acid desaturases in human adipose tissue: relationships between gene expression, desaturation indexes and insulin resistance, Diabetologia, 51 (2008) 328-335.

[150] R. Roberts, L. Hodson, A.L. Dennis, M.J. Neville, S.M. Humphreys, K.E. Harnden, K.J. Micklem, K.N. Frayn, Markers of de novo lipogenesis in adipose tissue: associations with small adipocytes and insulin sensitivity in humans, Diabetologia, 52 (2009) 882-890.

[151] J. Gong, H. Campos, S. McGarvey, Z. Wu, R. Goldberg, A. Baylin, Adipose tissue palmitoleic acid and obesity in humans: does it behave as a lipokine?, Am J Clin Nutr, 93 (2011) 186-191.

[152] M.M. Ibrahim, Subcutaneous and visceral adipose tissue: structural and functional differences, Obes Rev, 11 (2010) 11-18.

[153] J.M. Bruun, A.S. Lihn, S.B. Pedersen, B. Richelsen, Monocyte chemoattractant protein-1 release is higher in visceral than subcutaneous human adipose tissue (AT): implication of macrophages resident in the AT, J Clin Endocrinol Metab, 90 (2005) 2282-2289.

[154] N. Lanthier, I.A. Leclercq, Adipose tissues as endocrine target organs, Best Pract Res Clin Gastroenterol, 28 (2014) 545-558.

[155] K.E. Pinnick, M.J. Neville, B.A. Fielding, K.N. Frayn, F. Karpe, L. Hodson, Gluteofemoral adipose tissue plays a major role in production of the lipokine palmitoleate in humans, Diabetes, 61 (2012) 1399-1403.

[156] G. Jiang, Z. Li, F. Liu, K. Ellsworth, Q. Dallas-Yang, M. Wu, J. Ronan, C. Esau, C. Murphy, D. Szalkowski, R. Bergeron, T. Doebber, B.B. Zhang, Prevention of obesity in mice by antisense oligonucleotide inhibitors of stearoyl-CoA desaturase-1, J Clin Invest, 115 (2005) 1030-1038. 
[157] J. Cedernaes, J. Alsiö, Å. Västermark, U. Risérus, H.B. Schiöth, Adipose tissue stearoylCoA desaturase 1 index is increased and linoleic acid is decreased in obesity-prone rats fed a high-fat diet, Lipids in Health and Disease, 12 (2013) 2-2.

[158] R. Gutierrez-Juarez, A. Pocai, C. Mulas, H. Ono, S. Bhanot, B.P. Monia, L. Rossetti, Critical role of stearoyl-CoA desaturase-1 (SCD1) in the onset of diet-induced hepatic insulin resistance, J Clin Invest, 116 (2006) 1686-1695.

[159] J.B. Flowers, M.E. Rabaglia, K.L. Schueler, M.T. Flowers, H. Lan, M.P. Keller, J.M. Ntambi, A.D. Attie, Loss of stearoyl-CoA desaturase-1 improves insulin sensitivity in lean mice but worsens diabetes in leptin-deficient obese mice, Diabetes, 56 (2007) 1228-1239.

[160] M.T. Flowers, L. Ade, M.S. Strable, J.M. Ntambi, Combined deletion of SCD1 from adipose tissue and liver does not protect mice from obesity, Journal of Lipid Research, 53 (2012) 1646-1653.

[161] S. Carobbio, R.M. Hagen, C.J. Lelliott, M. Slawik, G. Medina-Gomez, C.-Y. Tan, A. Sicard, H.J. Atherton, N. Barbarroja, M. Bjursell, M. Bohlooly-Y, S. Virtue, A. Tuthill, E. Lefai, M. Laville, T. Wu, R.V. Considine, H. Vidal, D. Langin, M. Oresic, F.J. Tinahones, J.M. Fernandez-Real, J.L. Griffin, J.K. Sethi, M. López, A. Vidal-Puig, Adaptive Changes of the Insig1/SREBP1/SCD1 Set Point Help Adipose Tissue to Cope With Increased Storage Demands of Obesity, Diabetes, 62 (2013) 3697-3708.

[162] M. Malodobra-Mazur, A. Dziewulska, K. Kozinski, P. Dobrzyn, K. Kolczynska, J. Janikiewicz, A. Dobrzyn, Stearoyl-CoA desaturase regulates inflammatory gene expression by changing DNA methylation level in 3T3 adipocytes, Int J Biochem Cell Biol, 55 (2014) 40-50.

[163] E. Kim, J.-H. Lee, J.M. Ntambi, C.-K. Hyun, Inhibition of stearoyl-CoA desaturase1 activates AMPK and exhibits beneficial lipid metabolic effects in vitro, European Journal of Pharmacology, 672 (2011) 38-44.

[164] A. Koeberle, H. Shindou, T. Harayama, T. Shimizu, Palmitoleate is a mitogen, formed upon stimulation with growth factors, and converted to palmitoleoyl-phosphatidylinositol, J Biol Chem, 287 (2012) 27244-27254.

[165] A. Koeberle, C. Pergola, H. Shindou, S.C. Koeberle, T. Shimizu, S.A. Laufer, O. Werz, Role of p38 mitogen-activated protein kinase in linking stearoyl-CoA desaturase-1 activity with endoplasmic reticulum homeostasis, FASEB J, (2015).

[166] A.K. Kadegowda, T.A. Burns, S.L. Pratt, S.K. Duckett, Inhibition of stearoyl-CoA desaturase 1 reduces lipogenesis in primary bovine adipocytes, Lipids, 48 (2013) 967-976.

[167] F.E. Gomez, D.E. Bauman, J.M. Ntambi, B.G. Fox, Effects of sterculic acid on stearoylCoA desaturase in differentiating 3T3-L1 adipocytes, Biochem Biophys Res Commun, 300 (2003) 316-326.

[168] Y. Choi, Y.C. Kim, Y.B. Han, Y. Park, M.W. Pariza, J.M. Ntambi, The trans-10,cis-12 isomer of conjugated linoleic acid downregulates stearoyl-CoA desaturase 1 gene expression in 3T3-L1 adipocytes, J Nutr, 130 (2000) 1920-1924.

[169] A. Dobrzyn, J.M. Ntambi, Stearoyl-CoA desaturase as a new drug target for obesity treatment, Obes Rev, 6 (2005) 169-174.

[170] K. Clement, Bariatric surgery, adipose tissue and gut microbiota, Int J Obes (Lond), 35 Suppl 3 (2011) S7-15.

[171] S. Ledoux, I. Queguiner, S. Msika, S. Calderari, P. Rufat, J.M. Gasc, P. Corvol, E. Larger, Angiogenesis associated with visceral and subcutaneous adipose tissue in severe human obesity, Diabetes, 57 (2008) 3247-3257. 
[172] C.D. Green, C.G. Ozguden-Akkoc, Y. Wang, D.B. Jump, L.K. Olson, Role of fatty acid elongases in determination of de novo synthesized monounsaturated fatty acid species, J Lipid Res, 51 (2010) 1871-1877.

[173] E.G. Bligh, W.J. Dyer, A rapid method of total lipid extraction and purification, Can J Biochem Physiol, 37 (1959) 911-917.

[174] L.D. Roberts, S. Virtue, A. Vidal-Puig, A.W. Nicholls, J.L. Griffin, Metabolic phenotyping of a model of adipocyte differentiation, Physiol Genomics, 39 (2009) 109-119.

[175] S.A. Reza-Lopez, G.H. Anderson, I.M. Szeto, A.Y. Taha, D.W. Ma, High vitamin intake by Wistar rats during pregnancy alters tissue fatty acid concentration in the offspring fed an obesogenic diet, Metabolism, 58 (2009) 722-730.

[176] C. Fernandez, K. Schuhmann, R. Herzog, B. Fielding, K. Frayn, A. Shevchenko, P. James, C. Holm, K. Strom, Altered desaturation and elongation of fatty acids in hormone-sensitive lipase null mice, PLoS One, 6 (2011) e21603.

[177] E. Saito, T. Okada, Y. Abe, Y. Kuromori, M. Miyashita, F. Iwata, M. Hara, M. Ayusawa, H. Mugishima, Y. Kitamura, Docosahexaenoic acid content in plasma phospholipids and desaturase indices in obese children, J Atheroscler Thromb, 18 (2011) 345-350.

[178] R.C. Gentleman, V.J. Carey, D.M. Bates, B. Bolstad, M. Dettling, S. Dudoit, B. Ellis, L. Gautier, Y. Ge, J. Gentry, K. Hornik, T. Hothorn, W. Huber, S. Iacus, R. Irizarry, F. Leisch, C. Li, M. Maechler, A.J. Rossini, G. Sawitzki, C. Smith, G. Smyth, L. Tierney, J.Y. Yang, J. Zhang, Bioconductor: open software development for computational biology and bioinformatics, Genome Biol, 5 (2004) R80.

[179] R.C. Team, R: A language and environment for statistical computing., in, R Foundation for Statistical Computing, Vienna, Austria, 2013.

[180] B. Carvalho, H. Bengtsson, T.P. Speed, R.A. Irizarry, Exploration, normalization, and genotype calls of high-density oligonucleotide SNP array data, Biostatistics, 8 (2007) 485-499.

[181] P. McNicholas, T. Murphy, Parsimonious Gaussian mixture models, Statistics and Computing, 18 (2008) 285-296.

[182] P.D. McNicholas, K.R. Jampani, A.F. McDaid, T.B. Murphy, L. Banks, pgmm: Parsimonious Gaussian Mixture Models. R package version 1.0., in, 2011.

[183] P.D. McNicholas, T.B. Murphy, Model-based clustering of microarray expression data via latent Gaussian mixture models, Bioinformatics, 26 (2010) 2705-2712.

[184] J. MacQueen, Some methods for classification and analysis of multivariate observations, in: Fifth Berkeley Symposium on Mathematical Statistics and Probability, University of California, Los Angeles, 1967, pp. 281-297.

[185] L. Kaufman, P. Rousseeuw, Clustering by Means of Medoids, in Statistical Data Analysis Based on the L1-Norm and Related Methods, Y. Dodge, North-Holland, 1987.

[186] C. Fraley, A.E. Raftery, Model-Based Clustering, Discriminant Analysis, and Density Estimation, Journal of the American Statistical Association, 97 (2002) 611-631.

[187] G. Celeux, G. Govaert, Gaussian parsimonious clustering models, Pattern Recognition, 28 (1995) 781-793.

[188] C. Fraley, A. Raftery, L. Scrucca, mclust: Normal Mixture Modeling for Model-Based Clustering,Classification, and Density Estimation. R package version 4.2, in, 2013.

[189] J. Kusunoki, A. Kanatani, D. Moller, Modulation of fatty acid metabolism as a potential approach to the treatment of obesity and the metabolic syndrome, Endocrine, 29 (2006) 91-100.

[190] D.M. Mutch, Identifying regulatory hubs in obesity with nutrigenomics, Current Opinion in Endocrinology and Diabetes, 13 (2006) 431-437. 
[191] M.B. Fessler, L.L. Rudel, J.M. Brown, Toll-like receptor signaling links dietary fatty acids to the metabolic syndrome, Curr Opin Lipidol, 20 (2009) 379-385.

[192] R.L. Bradley, F.F. Fisher, E. Maratos-Flier, Dietary fatty acids differentially regulate production of TNF-alpha and IL-10 by murine 3T3-L1 adipocytes, Obesity (Silver Spring), 16 (2008) 938-944.

[193] X. Liu, M.S. Strable, J.M. Ntambi, Stearoyl CoA desaturase 1: role in cellular inflammation and stress, Adv Nutr, 2 (2011) 15-22.

[194] K.A. Marks, A.P. Kitson, K.D. Stark, Hepatic and plasma sex differences in saturated and monounsaturated fatty acids are associated with differences in expression of elongase 6 , but not stearoyl-CoA desaturase in Sprague-Dawley rats, Genes Nutr, 8 (2013) 317-327.

[195] S.M. Rahman, A. Dobrzyn, P. Dobrzyn, S.H. Lee, M. Miyazaki, J.M. Ntambi, StearoylCoA desaturase 1 deficiency elevates insulin-signaling components and down-regulates proteintyrosine phosphatase 1B in muscle, Proc Natl Acad Sci U S A, 100 (2003) 11110-11115.

[196] J.M. Ntambi, M. Miyazaki, Regulation of stearoyl-CoA desaturases and role in metabolism, Prog Lipid Res, 43 (2004) 91-104.

[197] E.D. Rosen, B.M. Spiegelman, What we talk about when we talk about fat, Cell, 156 (2014) 20-44.

[198] N. Halberg, I. Wernstedt-Asterholm, P.E. Scherer, The Adipocyte as an Endocrine Cell, Endocrinology and Metabolism Clinics of North America, 37 (2008) 753-768.

[199] J.M. Lenhard, Lipogenic enzymes as therapeutic targets for obesity and diabetes, Curr Pharm Des, 17 (2011) 325-331.

[200] J.B. Flowers, M.E. Rabaglia, K.L. Schueler, M.T. Flowers, H. Lan, M.P. Keller, J.M. Ntambi, A.D. Attie, Loss of Stearoyl-CoA Desaturase-1 Improves Insulin Sensitivity in Lean Mice but Worsens Diabetes in Leptin-Deficient Obese Mice, Diabetes, 56 (2007) 1228-1239.

[201] J.C. Ralston, F. Badoud, B. Cattrysse, P.D. McNicholas, D.M. Mutch, Inhibition of stearoyl-CoA desaturase-1 in differentiating 3T3-L1 preadipocytes upregulates elongase 6 and downregulates genes affecting triacylglycerol synthesis, Int J Obes (Lond), 38 (2014) 1449-1456. [202] R. Kasturi, V.C. Joshi, Hormonal regulation of stearoyl coenzyme A desaturase activity and lipogenesis during adipose conversion of 3T3-L1 cells, J Biol Chem, 257 (1982) 1222412230 .

[203] Y. Choi, Y. Park, M.W. Pariza, J.M. Ntambi, Regulation of Stearoyl-CoA Desaturase Activity by the trans-10,cis-12 Isomer of Conjugated Linoleic Acid in HepG2 Cells, Biochemical and Biophysical Research Communications, 284 (2001) 689-693.

[204] L.H. Storlien, A.B. Jenkins, D.J. Chisholm, W.S. Pascoe, S. Khouri, E.W. Kraegen, Influence of dietary fat composition on development of insulin resistance in rats. Relationship to muscle triglyceride and omega-3 fatty acids in muscle phospholipid, Diabetes, 40 (1991) 280289.

[205] B.M. Anderson, M.B. MacLennan, L.M. Hillyer, D.W. Ma, Lifelong exposure to n-3 PUFA affects pubertal mammary gland development, Appl Physiol Nutr Metab, 39 (2014) 699706.

[206] K.J. Livak, T.D. Schmittgen, Analysis of relative gene expression data using real-time quantitative PCR and the 2(-Delta Delta C(T)) Method, Methods, 25 (2001) 402-408.

[207] H. Alkhateeb, A. Chabowski, J.F. Glatz, B. Gurd, J.J. Luiken, A. Bonen, Restoring AS160 phosphorylation rescues skeletal muscle insulin resistance and fatty acid oxidation while not reducing intramuscular lipids, Am J Physiol Endocrinol Metab, 297 (2009) E1056-1066. 
[208] W.L. Holland, T.A. Knotts, J.A. Chavez, L.P. Wang, K.L. Hoehn, S.A. Summers, Lipid mediators of insulin resistance, Nutr Rev, 65 (2007) S39-46.

[209] O.A. MacDougald, M.D. Lane, Transcriptional regulation of gene expression during adipocyte differentiation, Annu Rev Biochem, 64 (1995) 345-373.

[210] J.A. Schmidt, G.M. Yvone, W.J. Brown, Membrane topology of human AGPAT3 (LPAAT3), Biochem Biophys Res Commun, 397 (2010) 661-667.

[211] M. Ishisaka, H. Hara, The roles of diacylglycerol kinases in the central nervous system: review of genetic studies in mice, J Pharmacol Sci, 124 (2014) 336-343.

[212] K. Takeuchi, K. Reue, Biochemistry, physiology, and genetics of GPAT, AGPAT, and lipin enzymes in triglyceride synthesis, Am J Physiol Endocrinol Metab, 296 (2009) E11951209.

[213] K.T. Landschulz, D.B. Jump, O.A. MacDougald, M.D. Lane, Transcriptional control of the stearoyl-CoA desaturase-1 gene by polyunsaturated fatty acids, Biochem Biophys Res Commun, 200 (1994) 763-768.

[214] S.J. van Dijk, E.J. Feskens, M.B. Bos, D.W. Hoelen, R. Heijligenberg, M.G. Bromhaar, L.C. de Groot, J.H. de Vries, M. Muller, L.A. Afman, A saturated fatty acid-rich diet induces an obesity-linked proinflammatory gene expression profile in adipose tissue of subjects at risk of metabolic syndrome, Am J Clin Nutr, 90 (2009) 1656-1664.

[215] W. Guo, S. Wong, W. Xie, T. Lei, Z. Luo, Palmitate modulates intracellular signaling, induces endoplasmic reticulum stress, and causes apoptosis in mouse 3T3-L1 and rat primary preadipocytes, Am J Physiol Endocrinol Metab, 293 (2007) E576-586.

[216] A. Dobrzyn, J.M. Ntambi, The role of stearoyl-CoA desaturase in body weight regulation, Trends Cardiovasc Med, 14 (2004) 77-81.

[217] E. Barber, A.J. Sinclair, D. Cameron-Smith, Comparative actions of omega-3 fatty acids on in-vitro lipid droplet formation, Prostaglandins Leukot Essent Fatty Acids, 89 (2013) 359366.

[218] J.C. Ralston, S. Matravadia, N. Gaudio, G.P. Holloway, D.M. Mutch, Polyunsaturated fatty acid regulation of adipocyte FADS1 and FADS2 expression and function, Obesity (Silver Spring), (2015).

[219] J. Folch, M. Lees, G.H. Sloane Stanley, A simple method for the isolation and purification of total lipides from animal tissues, J Biol Chem, 226 (1957) 497-509.

[220] A.C. Patterson, A.H. Metherel, R.M. Hanning, K.D. Stark, The percentage of DHA in erythrocytes can detect non-adherence to advice to increase EPA and DHA intakes, Br J Nutr, 111 (2014) 270-278.

[221] A.H. Metherel, J.J. Aristizabal Henao, K.D. Stark, EPA and DHA levels in whole blood decrease more rapidly when stored at -20 degrees $\mathrm{C}$ as compared with room temperature, 4 and 75 degrees C, Lipids, 48 (2013) 1079-1091.

[222] A.Y. Taha, A.H. Metherel, K.D. Stark, Comparative analysis of standardised and common modifications of methods for lipid extraction for the determination of fatty acids, Food Chemistry, 134 (2012) 427-433.

[223] H. Izadi, Development of a novel mass-selected internal positive chemical ionization quadrupole ion trap mass spectrometry technique for the quantitative analysis of isotpoic polyunsaturated fatty acids, in: Kinesiology, University of Waterloo, Waterloo, Ontario, Canada, 2009, pp. 65. 
[224] S. Sun, Y. Liu, J. Lu, A. Omar, Y. Bi, C. Wang, The inhibitory effects of PKCtheta on adiponectin expression is mediated by ERK in 3T3-L1 adipocytes, J Endocrinol Invest, 34 (2011) 8-15.

[225] A. Kennedy, K. Martinez, C.C. Chuang, K. LaPoint, M. McIntosh, Saturated fatty acidmediated inflammation and insulin resistance in adipose tissue: mechanisms of action and implications, J Nutr, 139 (2009) 1-4.

[226] M. Miyazaki, Y.-C. Kim, M.P. Gray-Keller, A.D. Attie, J.M. Ntambi, The Biosynthesis of Hepatic Cholesterol Esters and Triglycerides Is Impaired in Mice with a Disruption of the Gene for Stearoyl-CoA Desaturase 1, Journal of Biological Chemistry, 275 (2000) 30132-30138.

[227] H. Shi, M.V. Kokoeva, K. Inouye, I. Tzameli, H. Yin, J.S. Flier, TLR4 links innate immunity and fatty acid-induced insulin resistance, J Clin Invest, 116 (2006) 3015-3025.

[228] A. Gil, C. Maria Aguilera, M. Gil-Campos, R. Canete, Altered signalling and gene expression associated with the immune system and the inflammatory response in obesity, $\mathrm{Br} \mathrm{J}$ Nutr, 98 Suppl 1 (2007) S121-126.

[229] A.M. Manning, R.J. Davis, Targeting JNK for therapeutic benefit: from junk to gold?, Nat Rev Drug Discov, 2 (2003) 554-565.

[230] F. Bost, M. Aouadi, L. Caron, B. Binetruy, The role of MAPKs in adipocyte differentiation and obesity, Biochimie, 87 (2005) 51-56.

[231] E. Oh, M. Yun, S.K. Kim, G. Seo, J.S. Bae, K. Joo, G.T. Chae, S.B. Lee, Palmitate induces COX-2 expression via the sphingolipid pathway-mediated activation of NF-kappaB, p38, and ERK in human dermal fibroblasts, Arch Dermatol Res, 306 (2014) 339-345.

[232] H. Tilg, A.R. Moschen, Adipocytokines: mediators linking adipose tissue, inflammation and immunity, Nat Rev Immunol, 6 (2006) 772-783.

[233] M. Shimamura, M. Matsuda, Y. Ando, R. Koishi, H. Yasumo, H. Furukawa, I. Shimomura, Leptin and insulin down-regulate angiopoietin-like protein 3, a plasma triglycerideincreasing factor, Biochem Biophys Res Commun, 322 (2004) 1080-1085.

[234] R.K. Sera, J.H. McBride, S.A. Higgins, D.O. Rodgerson, Evaluation of reference ranges for fatty acids in serum, J Clin Lab Anal, 8 (1994) 81-85.

[235] J. Yin, Y. Wang, L. Gu, N. Fan, Y. Ma, Y. Peng, Palmitate induces endoplasmic reticulum stress and autophagy in mature adipocytes: Implications for apoptosis and inflammation, Int $\mathbf{J}$ Mol Med, (2015).

[236] T. Decker, P. Kovarik, Serine phosphorylation of STATs, Oncogene, 19 (2000) 26282637.

[237] L. Serrano-Marco, R. Rodriguez-Calvo, I. El Kochairi, X. Palomer, L. Michalik, W. Wahli, M. Vazquez-Carrera, Activation of peroxisome proliferator-activated receptor-beta/-delta (PPAR-beta/-delta) ameliorates insulin signaling and reduces SOCS3 levels by inhibiting STAT3 in interleukin-6-stimulated adipocytes, Diabetes, 60 (2011) 1990-1999.

[238] Y. Hamada, H. Nagasaki, A. Fujiya, Y. Seino, Q.L. Shang, T. Suzuki, H. Hashimoto, Y. Oiso, Involvement of de novo ceramide synthesis in pro-inflammatory adipokine secretion and adipocyte-macrophage interaction, J Nutr Biochem, 25 (2014) 1309-1316.

[239] K.M. Ajuwon, M.E. Spurlock, Palmitate activates the NF-kappaB transcription factor and induces IL-6 and TNFalpha expression in 3T3-L1 adipocytes, J Nutr, 135 (2005) 1841-1846.

[240] A.L. Dordevic, N. Konstantopoulos, D. Cameron-Smith, 3T3-L1 Preadipocytes Exhibit Heightened Monocyte-Chemoattractant Protein-1 Response to Acute Fatty Acid Exposure, PLoS ONE, 9 (2014) e99382. 
[241] A. Schaeffler, P. Gross, R. Buettner, C. Bollheimer, C. Buechler, M. Neumeier, A. Kopp, J. Schoelmerich, W. Falk, Fatty acid-induced induction of Toll-like receptor-4/nuclear factor- $\kappa \mathrm{B}$ pathway in adipocytes links nutritional signalling with innate immunity, Immunology, 126 (2009) 233-245.

[242] A. Baylin, M.K. Kim, A. Donovan-Palmer, X. Siles, L. Dougherty, P. Tocco, H. Campos, Fasting whole blood as a biomarker of essential fatty acid intake in epidemiologic studies: comparison with adipose tissue and plasma, Am J Epidemiol, 162 (2005) 373-381.

[243] L. Hodson, C.M. Skeaff, B.A. Fielding, Fatty acid composition of adipose tissue and blood in humans and its use as a biomarker of dietary intake, Prog Lipid Res, 47 (2008) 348-380.

[244] K.A. Harvey, C.L. Walker, Z. Xu, P. Whitley, T.M. Pavlina, M. Hise, G.P. Zaloga, R.A. Siddiqui, Oleic acid inhibits stearic acid-induced inhibition of cell growth and pro-inflammatory responses in human aortic endothelial cells, Journal of Lipid Research, 51 (2010) 3470-3480.

[245] E.K. Anderson, A.A. Hill, A.H. Hasty, Stearic acid accumulation in macrophages induces toll-like receptor 4/2-independent inflammation leading to endoplasmic reticulum stressmediated apoptosis, Arterioscler Thromb Vasc Biol, 32 (2012) 1687-1695.

[246] J.C. Ralston, D.M. Mutch, SCD1 inhibition during 3T3-L1 adipocyte differentiation remodels triacylglycerol, diacylglycerol and phospholipid fatty acid composition, Prostaglandins Leukot Essent Fatty Acids, (2015).

[247] G.P. Holloway, X.X. Han, S.S. Jain, A. Bonen, A. Chabowski, Chronic muscle stimulation improves insulin sensitivity while increasing subcellular lipid droplets and reducing selected diacylglycerol and ceramide species in obese Zucker rats, Diabetologia, 57 (2014) 832-840.

[248] J.A. Chavez, T.A. Knotts, L.P. Wang, G. Li, R.T. Dobrowsky, G.L. Florant, S.A. Summers, A role for ceramide, but not diacylglycerol, in the antagonism of insulin signal transduction by saturated fatty acids, J Biol Chem, 278 (2003) 10297-10303.

[249] L. Hodson, F. Karpe, Is there something special about palmitoleate?, Curr Opin Clin Nutr Metab Care, 16 (2013) 225-231.

[250] M. Ahmadian, R.E. Duncan, K. Jaworski, E. Sarkadi-Nagy, H.S. Sul, Triacylglycerol metabolism in adipose tissue, Future Lipidology, 2 (2007) 229-237.

[251] W. Samuel, R.K. Kutty, S. Nagineni, J.S. Gordon, S.M. Prouty, R.A. Chandraratna, B. Wiggert, Regulation of stearoyl coenzyme A desaturase expression in human retinal pigment epithelial cells by retinoic acid, J Biol Chem, 276 (2001) 28744-28750.

[252] S. Leekumjorn, H.J. Cho, Y. Wu, N.T. Wright, A.K. Sum, C. Chan, The Role of Fatty Acid Unsaturation in Minimizing Biophysical Changes on the Structure and Local Effects of Bilayer Membranes, Biochimica et biophysica acta, 1788 (2009) 1508-1516.

[253] E. Berlin, J.S. Hannah, K. Yamane, R.C. Peters, B.V. Howard, Fatty acid modification of membrane fluidity in Chinese hamster ovary (TR715-19) cells, Int J Biochem Cell Biol, 28 (1996) 1131-1139. 